University of Tennessee Health Science Center UTHSC Digital Commons

$12-2009$

\title{
Function and Pain in Community-Dwelling, Adult Mid-Southerners with Limb Loss
}

Cecile B. Evans

University of Tennessee Health Science Center

Follow this and additional works at: https://dc.uthsc.edu/dissertations

Part of the Nursing Commons

\section{Recommended Citation}

Evans, Cecile B. , "Function and Pain in Community-Dwelling, Adult Mid-Southerners with Limb Loss" (2009). Theses and Dissertations (ETD). Paper 77. http://dx.doi.org/10.21007/etd.cghs.2009.0086.

This Dissertation is brought to you for free and open access by the College of Graduate Health Sciences at UTHSC Digital Commons. It has been accepted for inclusion in Theses and Dissertations (ETD) by an authorized administrator of UTHSC Digital Commons. For more information, please contact jwelch30@uthsc.edu. 


\title{
Function and Pain in Community-Dwelling, Adult Mid-Southerners with Limb Loss
}

\begin{abstract}
Background: Limb loss affects about 1 in 190 people in the US. Risk factors for limb loss include diabetes, obesity, smoking, minority race, and poverty, which are all common in the Mid-South population. Function is impaired by limb loss, and pain associated with limb loss may further impair function.

Purpose: Describe the function, pain, health status, and demographics of community-dwelling, adult MidSoutherners with limb loss. Estimate the relationship between function and pain, health status, and demographics of community-dwelling, adult Mid-Southerners with limb loss. Estimate the joint influences on function by pain, health status, and demographics of community-dwelling, adult Mid-Southerners with limb loss.

Methods: Adult community-dwelling Mid-Southerners with limb loss ( $n=61$; male 65.6\%; Non-White race/ ethnicity $31.1 \%$; mean age 51.6) were recruited from the Mid-South using a purposive snowball sampling technique. Study participants were administered a 1-hour verbal survey that included questions regarding demographics, health status, SIP68 total a generic measure of sickness-related dysfunction, and the LLIP12 total a measure of the specific impact of limb loss on function, and a comprehensive pain assessment of Phantom limb pain (PLP), Residual limb pain (RLP), and Other Pain (OP). Pain intensity was assessed with a 5 point verbal descriptor scale converted to a numeric scale of 0-4.
\end{abstract}

Results: The mean SIP68 was 19.7 (range 1-54) and the mean LLIP12 was 4.4 (range 0-12). The prevalence of pain was PLP $83.6 \%$, RLP $55.0 \%$, and OP $62.7 \%$, with mean usual pain intensity was PLP 1.1, RLP 0.8, and OP 1.1. The significant correlations of the total SIP68 with usual pain intensity was PLP 0.62 $(p \leq .001)$, and OP $0.39(p=.002)$. Three statistical models of pain, health status, and demographic variables predicted $46 \%-52 \%$ of the shared variance of global function, physical function, and psychosocial function.

Discussion: Unique contributions of the demographics included a Mid-South sample with representation of Non-White race/ethnicity, especially Blacks (21.3\%) as well as women. The pain results are similar to phantom limb and residual limb pain studies done over the past three decades which show that there has been little progress in alleviating the frequency or severity of pain associated with limb loss. The phantom limb pain intensity variables usual PLP and worst PLP were most closely related to function. Usual RLP intensity was related to global function impaired by limb loss only. The high correlations of current OP and usual OP and continuous OP with function suggest both acute and chronic pain at a site not associated with an amputation can impair function. Pain quality descriptors were related to function with tiring PLP, tight band PLP, tiring RLP, abnormal position RLP, and tight band RLP had close relationships. Both continuous PLP and OP were closely related to impaired function suggesting that chronic pain was more disabling that acute. In the statistical models, usual phantom limb pain intensity was the greatest predictor of global function and psychosocial function. Continuous PLP was the greatest predictor of physical function. The magnitude of the influence of usual PLP and continuous PLP show that phantom pain intensity and chronicity predict the greatest variance in function. Non-White race/ethnicity was an important predictor in all three statistical models.

\section{Document Type}

Dissertation

\section{Degree Name}

Doctor of Philosophy $(\mathrm{PhD})$ 


\section{Program}

Nursing

Research Advisor

Veronica F. Engle, PhD.

\section{Keywords}

Amputation, Function, Mid-South United States, Pain, Phantom Limb Pain, Residual Limb Pain

\section{Subject Categories}

Medicine and Health Sciences | Nursing 
FUNCTION AND PAIN IN COMMUNITY-DWELLING, ADULT MID-SOUTHERNERS WITH LIMB LOSS

\author{
A Dissertation \\ Presented for \\ The Graduate Studies Council \\ The University of Tennessee \\ Health Science Center \\ In Partial Fulfillment \\ Of the Requirements for the Degree \\ Doctor of Philosophy \\ From the University of Tennessee
}

By

Cecile B. Evans

December 2009 
Copyright (C) 2009 by Cecile B. Evans

All rights reserved 


\section{DEDICATION}

I dedicate this dissertation to the loving memory of my parents, Mary Meyers "Buddie" Evans and

LTC Belmont Stuart Evans, Jr. (US Army Retired) 


\section{ACKNOWLEDGEMENTS}

First I want to acknowledge and thank my Committee members. Dr. Veronica F. Engle, who chaired the committee and guided the development of this research project as well as the development of my program of research. Dr. Lawrence W. Faulkner whose expertise in occupational therapy provided guidance to learn about ambulating with prosthetic limbs and understanding gait challenges and changes in persons with limb loss. Dr Marshall J. Graney whose expertise in statistics and their interpretation provided guidance in translating my data in to a meaningful account of the relationships presented in this study. Dr. Margaret T. Hartig, a primary care clinician with knowledge of health policy, provided clinical and health policy guidance in the interpretation of this study. Dr. Richard A. Sherman, whose expertise in phantom limb pain provided guidance for this project and who has been a mentor to my clinical research career for decades.

I want to acknowledge and thank Shirley Hancock for her editorial expertise and assistance with this dissertation.

I want to acknowledge and thank Sherri and William Samuels, founders of Out on a Limb and advocates for persons with limb loss, for telling me their story and bringing to my attention the real life experience of limb loss in the Mid-South. Sherri Samuels was also a key person in recruiting for this study, and introduced me to the limb loss community and limb loss providers.

I want to acknowledge and thank all of the support groups and professionals that assisted me in recruiting. This study was possible only because of the volunteers and professionals that referred persons with limb loss for their participation. I also want to acknowledge and thank all of the study participants who donated their time and openly shared information with me to complete this dissertation.

I want to acknowledge the constant support and encouragement of the immediate and extended Burke Evans Family. My husband Bob gave me his steady and constant support and encouragement in every way to complete this dissertation. My son Dan provided editorial and emotional support. My son Brennan supported me by reminding me to stay cool and chill. My son Jack shared his childhood with me and was a wonderful distraction. My sister Meg provided care for Jack when I was not able to. And thanks to all of the other extended family members who believed in me and supported me on this dissertation. 


\begin{abstract}
Background: Limb loss affects about 1 in 190 people in the US. Risk factors for limb loss include diabetes, obesity, smoking, minority race, and poverty, which are all common in the Mid-South population. Function is impaired by limb loss, and pain associated with limb loss may further impair function.

Purpose: Describe the function, pain, health status, and demographics of communitydwelling, adult Mid-Southerners with limb loss. Estimate the relationship between function and pain, health status, and demographics of community-dwelling, adult MidSoutherners with limb loss. Estimate the joint influences on function by pain, health status, and demographics of community-dwelling, adult Mid-Southerners with limb loss.
\end{abstract}

Methods: Adult community-dwelling Mid-Southerners with limb loss ( $\mathrm{n}=61$; male $65.6 \%$; Non-White race/ethnicity $31.1 \%$; mean age 51.6) were recruited from the MidSouth using a purposive snowball sampling technique. Study participants were administered a 1-hour verbal survey that included questions regarding demographics, health status, SIP68 total a generic measure of sickness-related dysfunction, and the LLIP12 total a measure of the specific impact of limb loss on function, and a comprehensive pain assessment of Phantom limb pain (PLP), Residual limb pain (RLP), and Other Pain (OP). Pain intensity was assessed with a 5 point verbal descriptor scale converted to a numeric scale of $0-4$.

Results: The mean SIP68 was 19.7 (range 1-54) and the mean LLIP12 was 4.4 (range 0-12). The prevalence of pain was PLP 83.6\%, RLP 55.0\%, and OP $62.7 \%$, with mean usual pain intensity was PLP 1.1, RLP 0.8 , and OP 1.1. The significant correlations of the total SIP68 with usual pain intensity was PLP $0.62(p \leq .001)$, and OP $0.39(p=.002)$. Three statistical models of pain, health status, and demographic variables predicted $46 \%-52 \%$ of the shared variance of global function, physical function, and psychosocial function.

Discussion: Unique contributions of the demographics included a Mid-South sample with representation of Non-White race/ethnicity, especially Blacks $(21.3 \%)$ as well as women. The pain results are similar to phantom limb and residual limb pain studies done over the past three decades which show that there has been little progress in alleviating the frequency or severity of pain associated with limb loss. The phantom limb pain intensity variables usual PLP and worst PLP were most closely related to function. Usual RLP intensity was related to global function impaired by limb loss only. The high correlations of current OP and usual OP and continuous OP with function suggest both acute and chronic pain at a site not associated with an amputation can impair function. Pain quality descriptors were related to function with tiring PLP, tight band PLP, tiring RLP, abnormal position RLP, and tight band RLP had close relationships. Both continuous PLP and OP were closely related to impaired function suggesting that chronic pain was more disabling that acute. In the statistical 
models, usual phantom limb pain intensity was the greatest predictor of global function and psychosocial function. Continuous PLP was the greatest predictor of physical function. The magnitude of the influence of usual PLP and continuous PLP show that phantom pain intensity and chronicity predict the greatest variance in function. NonWhite race/ethnicity was an important predictor in all three statistical models. 


\section{TABLE OF CONTENTS}

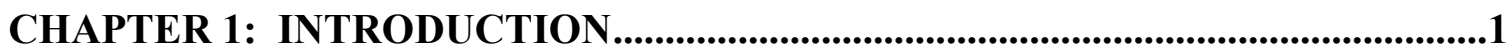

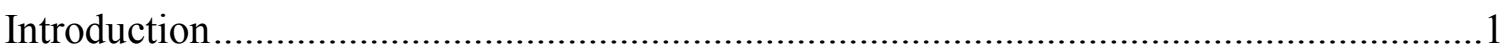

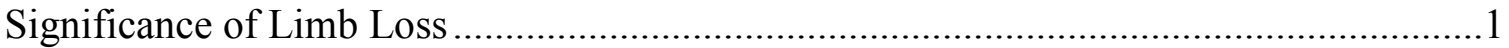

Function Impairment, Pain, Health Status, and Demographics in Persons

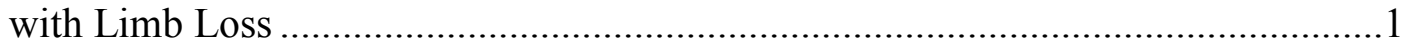

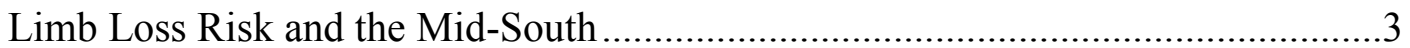

Societal Impact of Pain and Impaired Function in Persons with Limb Loss..............3

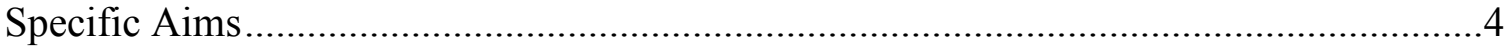

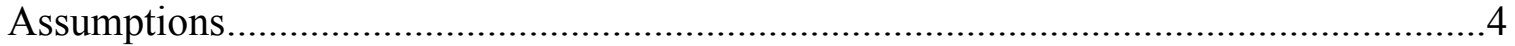

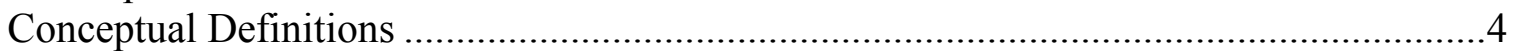

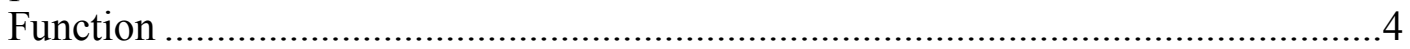

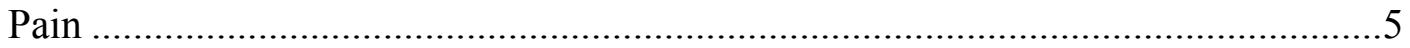

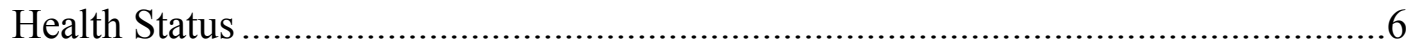

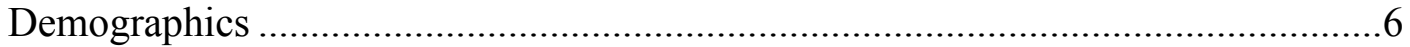

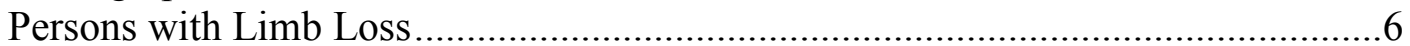

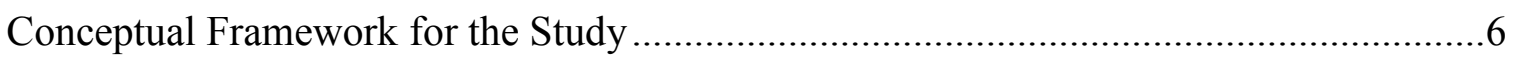

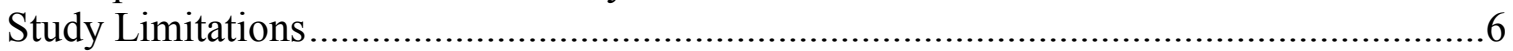

CHAPTER 2: REVIEW OF LITERATURE ...................................................................9

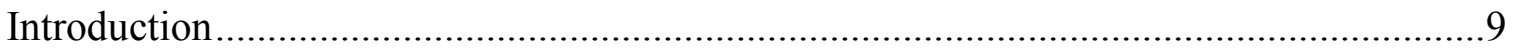

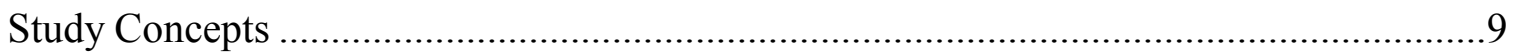

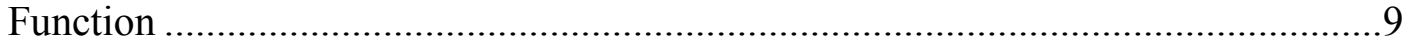

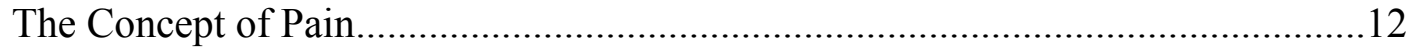

Pain by Location .........................................................................................

The Relationship of Function with Pain, Health Status, and Demographics .....................17

Relationships of Function and Pain .................................................................17

Relationship of Function and Health Status..........................................................19

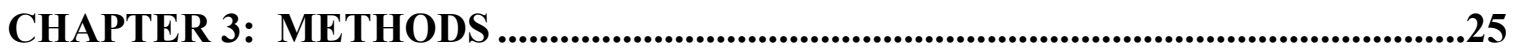

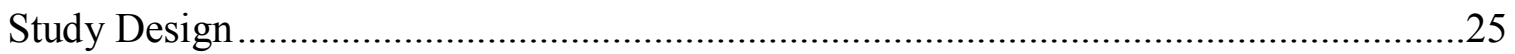

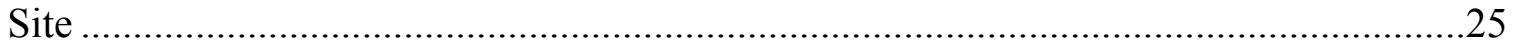

Sample

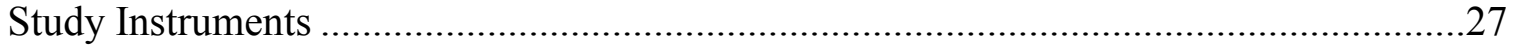

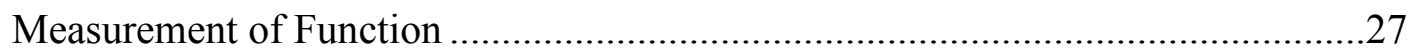

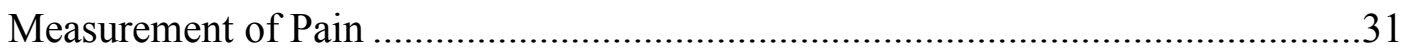

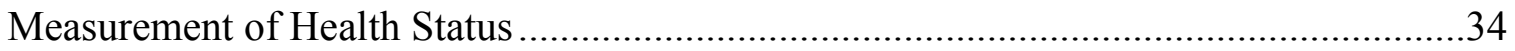

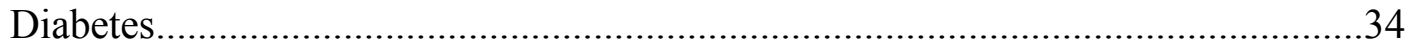

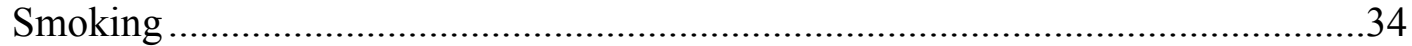

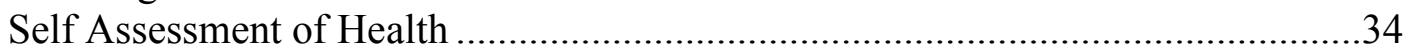

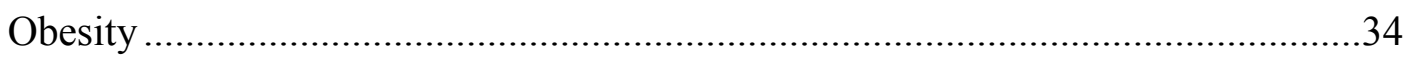

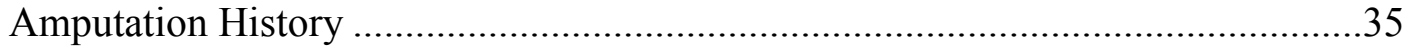

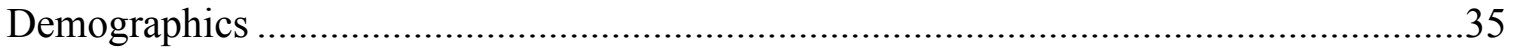




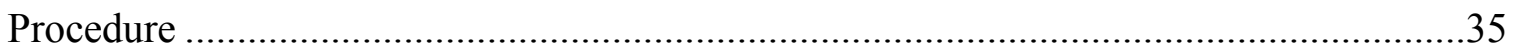

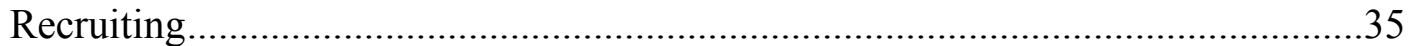

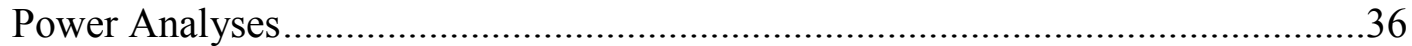

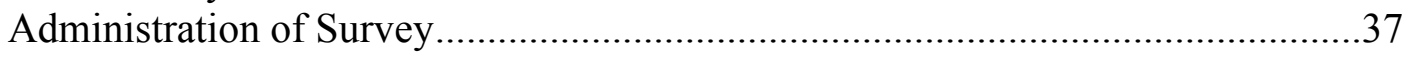

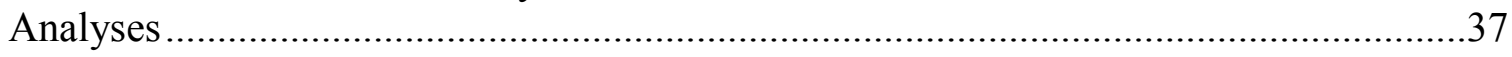

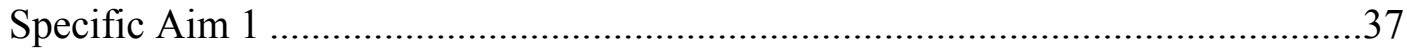

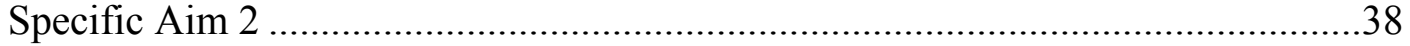

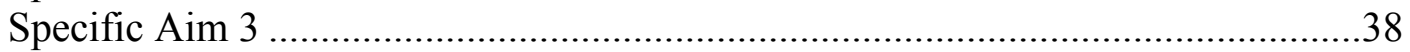

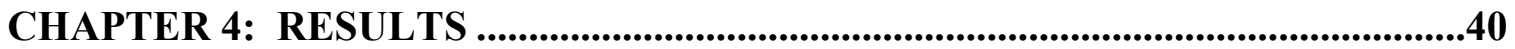

Sample

Internal Consistency of Function Variables..................................................................

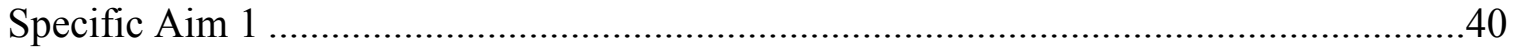

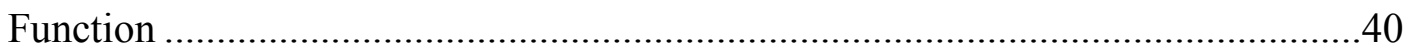

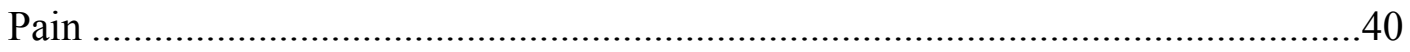

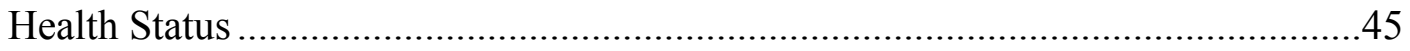

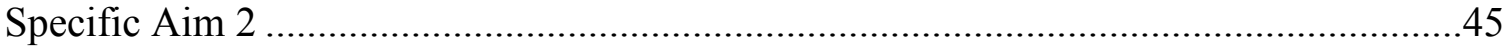

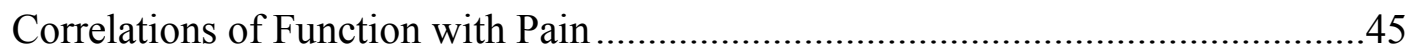

Correlations of Function with Health Status........................................................56

Correlations of Function with Demographics .......................................................60

Specific Aim 3 ................................................................................................60

Model to Predict Global Function (SIP68 Total)...................................................60

Model to Predict Physical Function (Mobility Range) ..............................................60

Model to Predict Psychosocial Function (Social Behavior) ........................................66

CHAPTER 5: DISCUSSION ...................................................................................68

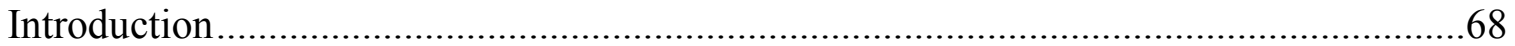

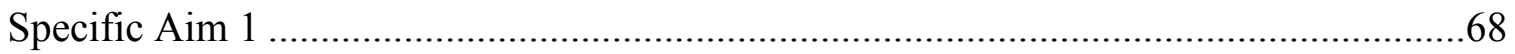

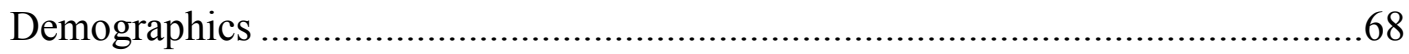

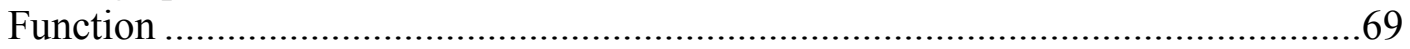

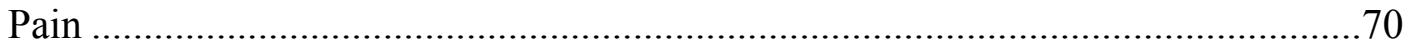

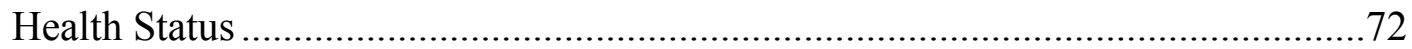

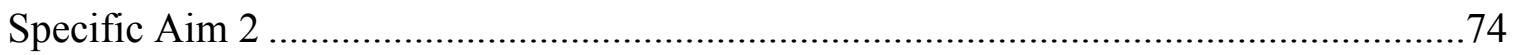

Correlations of Function and Pain ........................................................................74

Correlations of Function and Health Status ..........................................................77

Correlations of Function and Demographics ………..........................................79

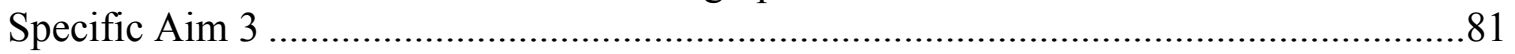

Model to Predict Global Function (SIP68 Total)...................................................81

Model to Predict Physical Function (Mobility Range) .............................................82

Model to Predict Psychosocial Function (Social Behavior) ......................................83

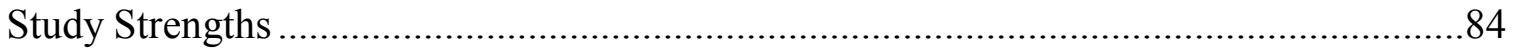

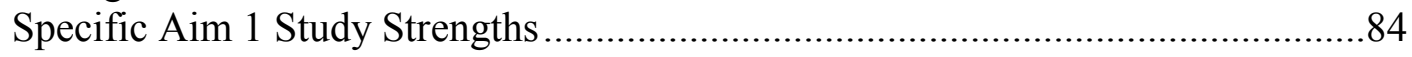

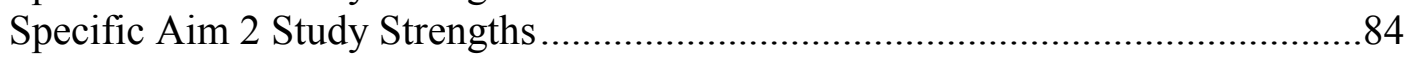

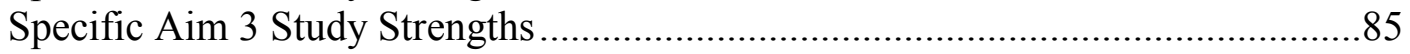

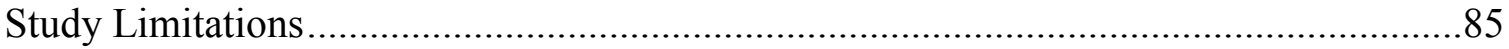




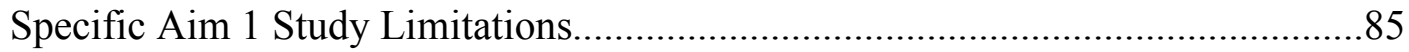

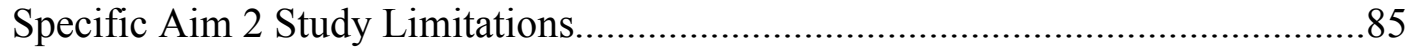

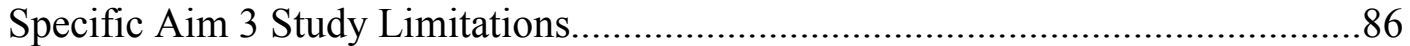

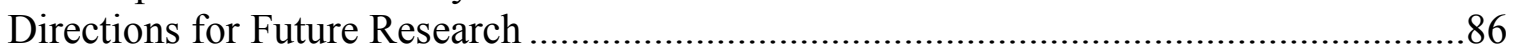

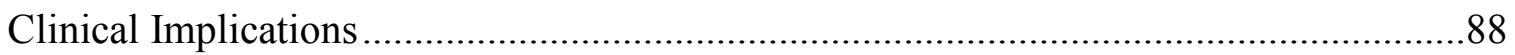

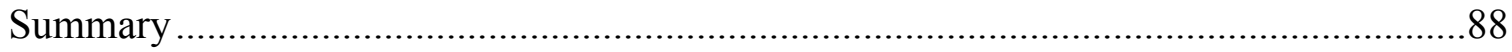

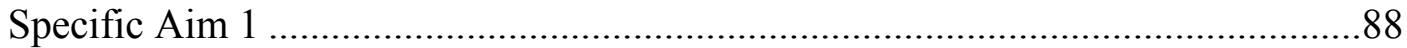

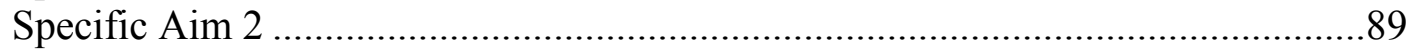

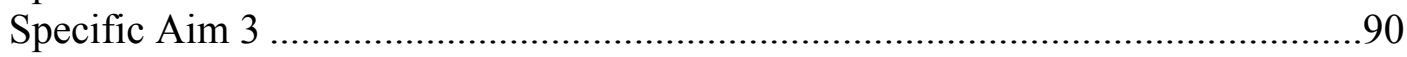

LIST OF REFERENCES..............................................................................................

APPENDIX A: HISTORY AND ETIOLOGIES OF PHANTOM LIMB PAIN ....101

APPENDIX B: UNIVERSITY OF TENNESSEE HEALTH SCIENCE

CENTER INSTITUTIONAL REVIEW BOARD APPROVAL ..................................104

APPENDIX C: VERBAL CONSENT..............................................................................110

APPENDIX D: STUDY INSTRUMENT .......................................................................111

APPENDIX E: DETAILED RECRUITING AND RECRUITMENT

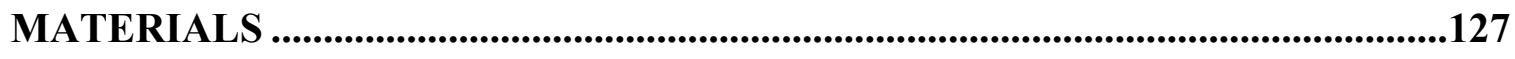

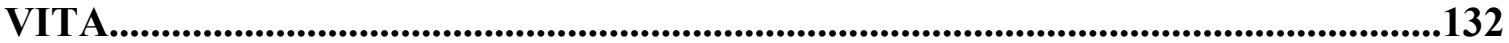




\section{LIST OF TABLES}

Table 4.1 Demographic Descriptive Data $(\mathrm{N}=61)$

Table 4.2 Cronbach's $\alpha$ for Global Function, Physical Function, and

Psychosocial Function Variables

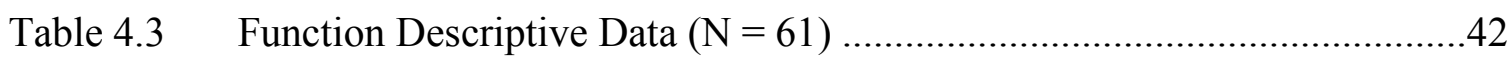

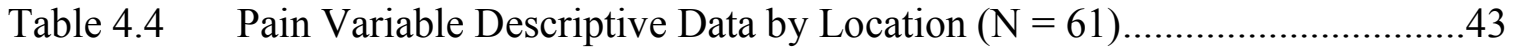

Table 4.5 Description of Pain Intensity Locations for Worst Other Pain

$(\mathrm{N}=59)$

Table 4.6 Health Status Descriptive Data $(\mathrm{N}=61)$

Table 4.7 Correlations of Global Function (SIP68 Total) with Pain Variables

by Pain Location

Table 4.8 Correlations of Global Function Attributed Specifically to Limb

Loss (LLIP12 Total) with Pain Variables by Pain Location.

Table 4.9 Correlations of Physical Function (Somatic Autonomy) with Pain

Variables by Pain Location

Table 4.10 Correlations of Physical Function (Mobility Control) with Pain

Variables by Pain Location

Table 4.11 Correlations of Physical Function (Mobility Range) with Pain

Variables by Pain Location

Table 4.12 Correlations of Psychosocial Function (Psychic Autonomy and

Communication) with Pain Variables by Pain Location

Table 4.13 Correlations of Psychosocial Function (Social Behavior) with Pain

Variables by Pain Location

Table 4.14 Correlations of SIP68 Psychosocial Function (Emotional Stability)

with Pain Variables by Pain Location.

Table 4.15 Correlations of Global Function (SIP68 Total and LLIP12 Total)

with Health Status Variables.

Table 4.16 Correlations of SIP68 Physical Function with Health Status

Variables and Number of Pain Areas .58 
Table 4.17 Correlations of SIP68 Psychosocial Function with Health Status

Variables

Table 4.18 Correlations of SIP68 Global Function Totals with Demographic Variables

Table 4.19 Correlations of SIP68 Physical Function with Demographic Variables

Table 4.20 Correlations of SIP68 Psychosocial Function with Demographic

Variables

Table 4.21 Regression with Dependent Variable Global Function (SIP68 Total)

$\left(\mathrm{R}^{2}=.52\right)$...

Table 4.22 Regression with Dependent Variable Physical Function

(Mobility Range) $\left(\mathrm{R}^{2}=.51\right)$

Table 4.23 Regression with Dependent Variable Psychosocial Function

(Social Behavior) $\left(\mathrm{R}^{2}=.46\right)$ 


\section{LIST OF ABBREVIATIONS}

AAF

ACA

AEA

AKA

BMI

BEA

CAST

ES

IRB

LLIP12

$\mathrm{MC}$

MR

OOAL

OP

PAC

PCA

PI

PLP

PPA

RLP

SA
American Amputee Foundation

Amputee Coalition of America

Above the Elbow Amputation Level

Above the Knee Amputation Level

Body Mass Index

Below the Elbow Amputation Level

Christian Amputee Support Team (Limb Loss Support Ministry)

SIP68: Psychosocial Function: Emotional Stability

Institutional Review Board

Limb Loss Impact Profile 12: Global Function

SIP68: Physical Function: Mobility Control

SIP68: Physical Function: Mobility Range

Out on a Limb (Limb Loss Support Group)

Other Pain

SIP68: Psychosocial Function: Psychic Autonomy and Communication

Principle Components Analyses

Principle Investigator

Phantom Limb Pain

Prosthetic Profile of the Amputee

Residual Limb Pain

SIP68: Physical Function: Somatic Autonomy 
SAH

SB

SF-36

SIP68

SIP136

UTHSC
Health Status: Self-Assessment of Health

SIP68: Psychosocial Function: Social Behavior

Short-Form 36 Health Survey

Sickness Impact Profile 68: Global Function

Sickness Impact Profile 136: Global Function

University of Tennessee Health Science Center 


\section{CHAPTER 1: INTRODUCTION}

\section{Introduction}

Limb loss is common, affecting about 1 in 190 persons in the United States based on estimates through 1999; prevalence is projected to double by the year 2050. ${ }^{1}$ The loss of a limb impairs function. ${ }^{2-10}$ Maintenance and improvement of function in persons with limb loss is important to the individual and society. The cost of a limb loss and the supportive care for the first two years that follow an amputation can reach over half a million dollars. ${ }^{11}$ Health care and supportive care that are associated with impaired function will continue to increase health care requirements and therefore health care costs.

Amputations attributed to vascular causes are responsible for this increase. ${ }^{12}$ Common conditions associated with vascular limb loss include diabetes, ${ }^{13}$ obesity, ${ }^{14}$ smoking, ${ }^{15}$ increased age, ${ }^{12}$ Non-White race/ethnicity, ${ }^{12}$ and poverty. ${ }^{16}$ The rise in the number of amputations is caused by societal changes that include increases in 1) the number of persons with diabetes; ${ }^{17}$ 2) obesity; ${ }^{18}$ ) aging of the United States population; ${ }^{19}$ and 4) the percentage of persons with Non-White race/ethnicity is increasing compared to Whites. ${ }^{20}$ Additionally, tobacco smoking remains common ${ }^{21}$ and the poverty rate remains stable at about $12 \%,{ }^{22}$ which are also associated with vascular limb loss.

Pain, including phantom limb pain, residual limb pain, and other pain, is common and occurs in most persons with limb loss. ${ }^{23-27}$ Pain-related disability associated with limb loss may also impair function. Pain with limb loss, especially phantom limb pain, frequently is not discussed by persons with limb loss with health care providers. ${ }^{28}$ The result is poorly assessed and under-treated pain, especially phantom limb pain, in persons with limb loss.

\section{Significance of Limb Loss}

\section{Function Impairment, Pain, Health Status, and Demographics in Persons with Limb Loss}

The number of persons with limb loss who are at risk for impaired function is increasing. Non-White minorities that have health disparities which put them at risk for limb loss are also at risk for under- treated pain. ${ }^{29-31}$ Non-White race/ethnicity is also associated with a greater level of pain-related disability. ${ }^{31}$ These disparities become augmented with very poorly treated phantom limb pain. The Non-White minority population is increasing at a faster rate than Whites. ${ }^{20}$ Since Non-White minorities have up to twice the risk for vascular amputation compared to Whites, there is a clinical need 
to understand the implications of amputation-related pain and disability in Non-White minority persons with limb loss.

Poverty alone is a risk factor for limb loss. ${ }^{16}$ The number of persons without insurance in the United States is increasing. ${ }^{22}$ Therefore, the number of persons at risk for access to care adequate health care is increasing. Low socioeconomic status was shown in a study to be as important as race/ethnicity in pain treatment and pain-related disability. ${ }^{32}$ Thus it is extremely important to include persons with low socioeconomic status in a study of amputation-related pain and disability.

A study reported that persons with limb loss usually changed occupations after an amputation and that unemployment was associated with high phantom limb pain intensity. ${ }^{33}$ This scenario could spiral into loss of income, impaired function, and undertreated pain. As income decreases, there can be an accompanying decrease in access to health care, pain treatment, therapies, and prosthetic device maintenance.

The combined effects of low health literacy and poor clinical pain assessment and pain treatment set the stage for increased pain. Low health literacy, associated with lower education and poverty, is an independent risk factor for impaired function. ${ }^{34}$ This leads to personal suffering associated with untreated pain and the impairment of function. Impaired function affects the individual with limb loss on a personal level as well as the health care system and other social supports.

The health status and demographics that influence health disparities which increase limb loss risk also increase risk for decreased function that may be related to inadequate pain treatment. Phantom limb pain is common with limb loss yet remains poorly treated. ${ }^{35}$ Patient education related to phantom limb pain is not a standard of perioperative nursing practice. Persons with limb loss receive no or little information about phantom limb pain and are often shocked by the experience. Phantom limb pain does not respond to opiates in all persons affected, ${ }^{36}$ so treatment may require complementary and alternative interventions. Primary care providers frequently have a poor understanding of phantom limb pain which, combined with a reluctance of phantom limb pain sufferers to discuss it with them, inhibits phantom limb pain assessment and treatment. ${ }^{28}$ Untreated phantom limb pain in the peri-operative period, recovery phase, or at a later time, can lead to chronic phantom limb pain that can cause persistent suffering ${ }^{37}$ that in turn may impair function throughout the lifespan.

Impaired function may be exacerbated by untreated pain. There is an immediate need to describe function and pain in persons with limb loss that represent a growing segment of the population that is at the greatest risk for limb loss. Non-White minorities and persons from all socioeconomic strata need to be included in these studies, as these demographics will have the highest rates of limb loss and greatest health disparities that prevent adequate pain treatment. 


\section{Limb Loss Risk and the Mid-South}

The Mid-South states (i.e., Alabama, Arkansas, Kentucky, Mississippi, and Tennessee) are among the states with very high rates of limb loss risk factors. These states include the highest rates of diabetes, ${ }^{38}$ obesity, ${ }^{38}$ smoking, ${ }^{38}$ and concentrated poverty $^{39}$ in the United States. The Mid-South states have a large representation of African Americans, and the Southern United States is projected to be home to over 64\% of all African Americans in the living in the United States by $2025 .^{20}$ These factors, combined, place Mid-Southerners at a high risk for having the health status and demographics which lead to an amputation from vascular causes. Despite that fact that the Mid-South has many limb loss risk factors, it is not known if studies that include mostly White, middle class, educated samples can be generalized to communitydwelling, adult Mid-Southerners with limb loss.

The study of limb loss in the Mid-South will provide an overview of the outcome of limb amputation, which is an unfortunate consequence of growing health status trends in the United States. The Mid-South provides a population with the health status and demographic profile today which represents national trends that will affect the United States in the future. Since function and pain with limb loss have not been studied in the Mid-South, it is not known if data from previous studies can be generalized to community-dwelling, adult Mid-Southerners with limb loss.

\section{Societal Impact of Pain and Impaired Function in Persons with Limb Loss}

An increase in the number of persons with limb loss will have a significant impact on the health care system. This impact will be greater if function is not maintained or improved after an amputation. Improving and maintaining function in persons with limb loss is vital to individuals, families, the health care system, and social support services.

Individuals and families suffer when a family member has pain and decreased function from a disability. ${ }^{40}$ Everyday life of individuals and families changes when a person with limb loss has their ability to perform activities of daily living interrupted. Impairment of physical function includes the ability to perform basic self-care and have mobility; it increases the need for assistance from others or assistive devices. An impairment of psychosocial function affects mood and vital social relationships. A person's productive role in society can be lost or affected so that the role of providing financial support or care giving for themselves or others is lost.

Health care and social support systems will be affected by an increase in the number of persons with amputations, and impaired function in persons with limb loss will exacerbate the impact. ${ }^{11}$ When function is impaired in persons with limb loss, there is an increase in utilizing the health care system resources accompanied by a decrease in the ability to pay for services and/or maintain health care insurance. The trend toward providing universal health care for all Americans will mean that government sponsored 
health care will bear the financial impact of these resources. This impact will affect society as a whole.

\section{Specific Aims}

Following are the three specific aims of this study:

- Specific Aim 1: Describe the function, pain, health status, and demographics of community-dwelling, adult Mid-Southerners with limb loss.

- Specific Aim 2: Estimate the relationship between function and pain, health status, and demographics of community-dwelling, adult Mid-Southerners with limb loss.

- Specific Aim 3: Estimate the joint influences on function by pain, health status, and demographics of community-dwelling, adult Mid-Southerners with limb loss.

\section{Assumptions}

1. All study participants living in the Mid-South share a similar culture and access to health care. This is influenced by demographics associated with health disparities including race/ethnicity and socioeconomic status.

2. Persons with limb loss are able to accurately describe function, pain, health status, and demographics.

3. Change in function is influenced by pain, health status, and demographics.

4. Persons with limb loss experience function impairments regardless of type of limb loss - whether upper extremity or lower extremity.

5. Function may be impaired with or without pain.

\section{Conceptual Definitions}

\section{Function}

Function is "the action of an organism or system." " It is independent of health or illness. Function describes the daily achievements one is able to perform. The measure of function quantifies an individual's adaptive skills to perform in their environment independently. Function is an outcome that elicits a snapshot into the real lives of individuals, including daily physical and psychosocial performance. 


\section{Global Function}

Global function refers the sum of all functions performed by an individual. It is a holistic appraisal of how one is able to perform all expected activities independently.

\section{Physical Function}

Physical function refers to all of the actions that involve physical tasks, such as performing self-care independently and mobility without assistance.

\section{Psychosocial Function}

Psychosocial function refers to all actions that require independent cognitive skills and social activities and relationships.

\section{Pain}

Pain was defined by the International Association for the Study of Pain as "an unpleasant sensory and emotional experience associated with actual or potential tissue damage or described in terms of such damage." 42 Pain is a construct with domains that include intensity, location, quality, and pattern.

\section{Pain Intensity} pain is.

Pain intensity is the quantity of pain. It is a measure of how much or how severe

\section{Pain Location}

Pain location is where the individual experiences pain.

\section{Pain Quality}

This is the unique sensation of how the pain feels to the individual.

\section{Pain Pattern}

This is the temporal pattern of pain and whether the pain is either intermittent, continuous, or both intermittent and continuous. 


\section{Health Status}

Health status is a representation of health factors that are related to limb loss risk, function impairment, or to limb loss history.

\section{Demographics}

Demographics included are related to limb loss risk or variations in function.

\section{Persons with Limb Loss}

"Persons with limb loss" is a term to represent anyone who is missing an arm or leg. This term was chosen to be consistent with patient-centered care. Patient-centered care delivery is defined as "recogniz[ing] the patient or designee as the source of control and full partner in providing compassionate and coordinated care based on respect for patient's preferences, values, and needs." 43

\section{Conceptual Framework for the Study}

Figure 1.1 provides a diagram of the conceptual framework for this study. The conceptual framework was defined by the PI with consultations from experts with experience in function, pain with limb loss, the Mid-South population health status and demographics. This framework guides the organization and analyses of this study.

\section{Study Limitations}

The limitations of this study were the following:

1. This was a cross-sectional study, so longitudinal function changes and the influence of pain, health status, and demographics over time were not assessed.

2. This was a quantitative study that did not evaluate the experience of limb loss.

3. All data were gathered by self-report. Subjective responses may not always be consistent with data in medical records, especially in persons with low health literacy. Physical measurements for items such as height and weight might increase validity in these areas. Subjective report does evaluate the subjective nature of function, pain, health status, and demographics.

4. The study had 61 participants. Despite the small sample size, there were highly significant relationships. On the items that were not found to be significant, the 


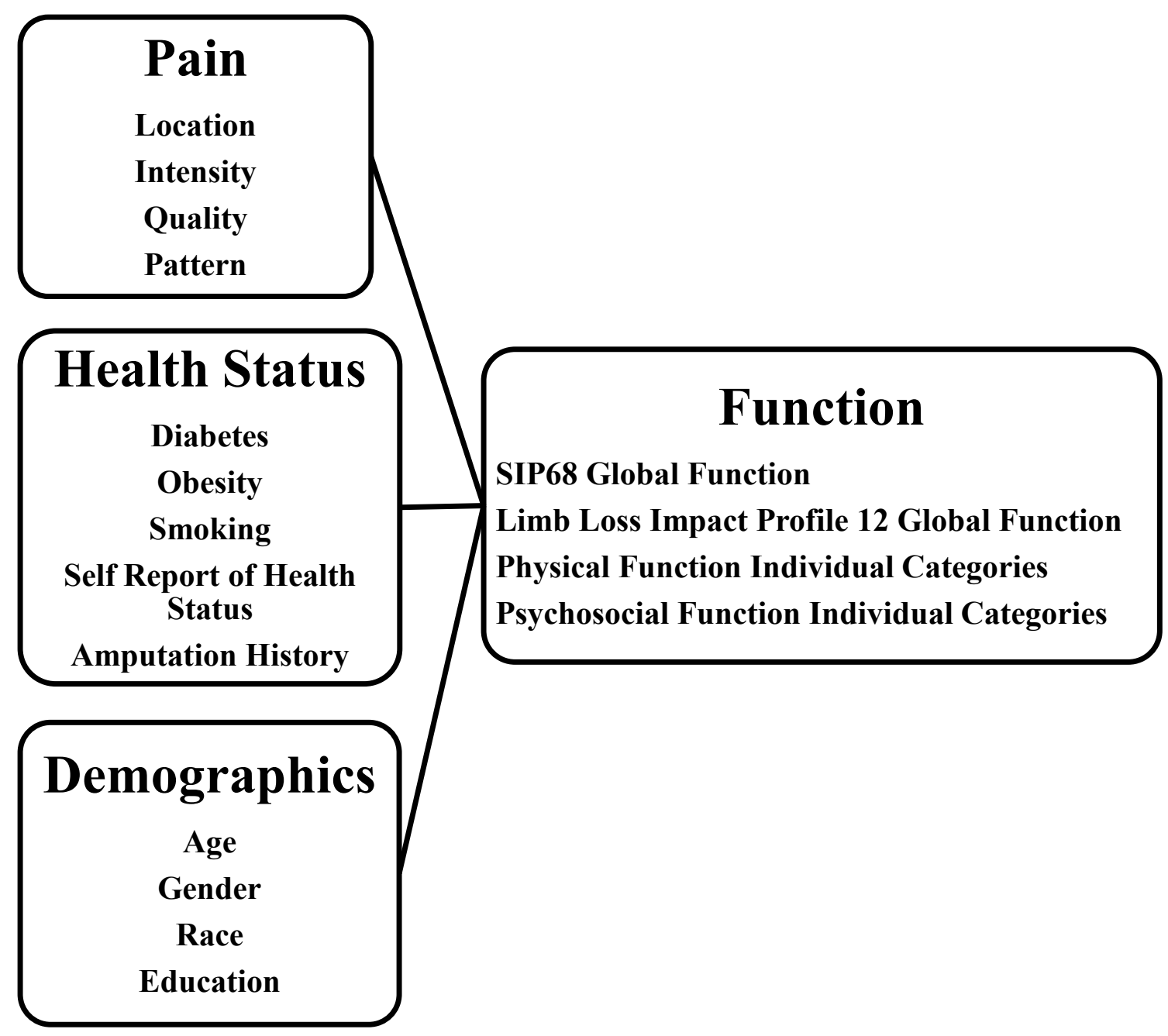

Figure 1.1 Conceptual Framework for Study 
findings may not fully explain all of the significant relationships, as there may be false negatives that do not reflect significant correlations.

5. There was wide variability in individual amputation histories, including number of limbs lost, type of limb loss, level of amputation, amputation duration, and cause of amputation. However, there were common experiences that influenced function across the limb loss population.

6. Generalizability of this study, which is diminished by the small sample size and restricted recruitment region, is limited to community-dwelling, White and NonWhite, adult Mid-Southerners with limb loss.

7. This study did not include persons with limb loss during the first six months after limb loss. It was assumed that pain and function were rapidly changing during the post-surgical recovery period.

8. Pain domains have a unique contribution to the pain experience. 


\section{CHAPTER 2: REVIEW OF LITERATURE}

\section{Introduction}

This chapter is a review of literature that will focus on function, pain, health status, and the demographics of persons with limb loss. The relationship of pain, health status, and demographics with function in persons with limb loss will follow. The literature search for the studies reviewed included the data bases Medline, Cinahl, PsychINFO, as well as the World Wide Web. The search included a minimum of 20 years of literature, and the literature review included older landmark studies. Older studies on the history and development of the study concepts were also included.

\section{Study Concepts}

\section{Function}

Function began as a very simple observation of physiology and has evolved into the study of complex human behaviors. This section on function will begin by discussing the historical concept of function. Function and limb loss will then be discussed. Function and limb loss is organized as global function, physical function, and psychosocial function.

\section{Overview of the Concept of Function}

Function is "the action of an organism or system." 41 Function is the action performed by part of a living system or by the complete individual. An action is "something done or performed." 44 Because function is an action, it is unique to who or what is performing that function. Function can range from simple activities, such as breathing, to complex activities, such as altruistic behavior. ${ }^{45}$ Function includes all tasks, from basic activities of daily living to active participation in occupational or recreational activities. ${ }^{46}$ A task is "a definite piece of work assigned to, falling to, or expected of a person." 47

In the United States, the first documented studies of a human function was by a surgeon who studied the physiology of gastric function in the 1820's by observing the digestive system. ${ }^{48}$ Human behavior was described to be a function by the philosopher Herbert Spencer in the late $19^{\text {th }}$ century. ${ }^{49}$ This behavioral view of function was expanded by William James, who described functions to include an anatomical and physiological perspective with an individual's adaptation to his or her environment, and the interaction of his or her consciousness. ${ }^{49}$

The study of function by observing human capabilities and quantifying these measures and tests of simple mental tasks was started by James Cattel in the early $20^{\text {th }}$ 
century. ${ }^{50}$ In the $1940 \mathrm{~s}$, measuring function became an interest for gerontologists, who were interested in the assessment of older adults' independence. ${ }^{51}$ This interest continued into the 1960s and 1970s, and many instruments were developed for older adults who had changing needs that dictated whether they lived independently in the community or needed more intense daily assistance. ${ }^{52,53}$ The development of the modern function instruments include a measure of independence. Independence is highly valued in Western culture, but may not reflect the values of an individual with changing abilities. Instruments to assess the ability to perform activities of daily living independently were developed at this time specifically for the chronically ill and older adults. ${ }^{54}$ Function measurement included physical health, quality of self-maintenance, quality of role activity, intellectual status, social activity, attitude toward the world and self, and emotional status. ${ }^{46}$ Function measurements today are a way to quantify how a person's activities deviate from an expected standard or norm and show functional impairments from the expected norm. 55 A norm can be defined as "a standard, model, or pattern."

Function is separate and distinct from the diagnoses of a specific disease or impairment. ${ }^{57}$ A level of sickness may affect and change one's level of functioning significantly, independent of the seriousness of the disease prognosis. Function includes adaptive behavior to one's specific environment ${ }^{53}$ and should include subjective self reports about this adaptation. ${ }^{57}$

Global function describes an individual's complete ability to perform all expected behaviors. Global function includes a wide diversity of individual experiences which are the culmination of physical and psychosocial action and abilities. ${ }^{55}$ The two domains of global function, physical function and psychosocial function as measured by the Sickness Impact Profile 136 (SIP136), each have unique contributions to function. ${ }^{58}$

In summary, function is performing roles that society expects from all individuals. The definition of a role includes "the rights, obligations, and expected behavior patterns associated with a particular social status." ${ }^{59}$ A deviation from the ability to perform these expected roles is impaired function. Global function includes the individual and combined contribution of physical and psychosocial domains. It is known that the measure of function is an assessment of one's impairment in abilities. Modern assessment of function does not include the measurement of specific strengths and skills that allow individuals to adapt to their environment in situations that deviate from an expected norm.

\section{Global Function in Persons with Limb Loss}

Previous studies reported that global function, including the domains of physical function and psychosocial function, is impaired in persons with limb loss. These studies measured global function with the Sickness Impact Profile 68 (SIP68) in persons with limb loss from the Netherlands. ${ }^{2,3,60}$ Studies of persons with limb loss have reported impaired global function using the SIP136 in persons with limb loss from the Lower Extremity Assessment Project (LEAP), ${ }^{4,10}$ persons with diabetes and vascular limb 
loss from Texas, ${ }^{6}$ and persons with limb loss from the Netherlands. ${ }^{7,9}$ Participants in the LEAP study were male $81.5 \%$, White $73.0 \%$, Black $19.7 \%$, and other race/ethnicity $7.3 \%$, with over half under age 35 years who had undergone traumatic amputations at one of eight level one trauma centers throughout the United States. ${ }^{61}$ Global function was reported to be impaired in persons with limb loss by studies using other subjective assessments of global function. This includes samples of persons with upper extremity limb loss from the United Kingdom, ${ }^{62}$ lower extremity limb loss from Sri Lanka, ${ }^{63}$ and persons with any limb loss from Washington state, combined with national advertisement respondents (male 68.2\%, White 89.5\%), ${ }^{64}$ and persons with lower limb loss from Illinois ${ }^{65}$ and Washington state. ${ }^{66}$

In summary, it is known that limb loss is associated with impairment of global function. When global function was assessed, all of the studies reported impairment in global function, regardless of the instrument or population. The LEAP study, which included minorities from throughout the United States, had a population that was young and mostly male, with traumatic limb loss only. Women have been under-represented in studies of global function in persons with limb loss. Previous studies that used the SIP68 to measure global function studied only persons with limb loss from the Netherlands. Other studies of global function with limb loss that used subjective instruments other than the SIP136 or the SIP68 had samples from outside of the United States or in geographic areas that are not representative of the Mid-South population. It is not known if the results from these studies can be generalized to adult community-dwelling, adult Mid-Southerners with limb loss. Additionally, it was not identified how persons with limb loss reported impaired global function that was attributed specifically to limb loss.

\section{Physical Function in Persons with Limb Loss}

Physical function impairment is well documented in persons with limb loss. Physical impairment was demonstrated with the SIP68 categories somatic autonomy, mobility control, and mobility range in persons with limb loss from the Netherlands. ${ }^{3}$ Mobility control is the ability to control one's body, or what one's true ability is and mobility range is the ability to perform expected tasks, or the physical activities that an individual is actually performing ${ }^{67}$. The mean physical function sub-scores of the SIP136 showed impairment in LEAP study participants, ${ }^{4,5,10}$ in persons with limb loss from Texas with diabetes and vascular limb loss, ${ }^{6}$ and in persons with limb loss recruited in Washington state. ${ }^{66}$ Other physical function measures of mobility show impairment in function with lower extremity limb loss. Mobility was impaired when measured through subjective assessment with the Prosthetic Evaluation Questionnaire, in persons with limb loss in Illinois, ${ }^{65}$ and the physical function sub-scale of the SF-36 in persons with traumatic limb loss from Maryland (male 87\%, White 75\%). ${ }^{68}$

In summary, it is known that there is physical function impairment in persons with limb loss. Mobility is impaired in persons with lower limb loss. The only study indentified that reported the sub-scores of the SIP68 in persons with limb loss had a sample from the Netherlands. Studies of physical function within the United States did 
not include samples that are representative of community-dwelling, adult MidSoutherners with limb loss. It is not known how impairment of physical function can be generalized to community-dwelling, adult Mid-Southerners with limb loss.

\section{Psychosocial Function in Persons with Limb Loss}

The literature is inconclusive about the effect of limb loss on psychosocial function in persons with limb loss. Impaired psychosocial function was reported as measured with the SIP68 categories of psychic autonomy and communication, social behavior, and emotional stability in persons with limb loss from the Netherlands. ${ }^{3}$ Impaired psychosocial function was shown in LEAP study participants using the psychosocial subscales of the SIP136. ${ }^{4,5,10}$ However, there was no difference reported between persons with diabetes recruited from Texas with or without limb loss in the psychosocial function sub-scales of the SIP136. ${ }^{6}$ This may have been because all the persons with diabetes in this sample showed psychosocial impairment with the psychosocial sub-scales of the SIP136 compared to the general population. This could have been because persons with diabetes experience impaired psychosocial function, such as depression associated with their diabetes. ${ }^{69}$

Studies have reported no decrease in psychosocial function measures attributed specifically to limb loss as indicated by the psychosocial function subscales of the SF-36 compared to similar populations without limb loss from Washington state ${ }^{66}$ and Vietnam veterans. $^{70,71}$ This suggests that the subscales of the SF-36 may not have the sensitivity to detect psychosocial impairment in persons with limb loss. Psychosocial scores on the Prosthetic Evaluation Questionnaire, which was validated on the SF-36, revealed less psychosocial impairment compared to physical impairment in persons with limb loss from Illinois. ${ }^{65}$

It is known that studies of psychosocial function in persons with limb loss are inconclusive and may be reflective of the instrument used to study function. Studies that used the SIP68 or SIP136 showed impairment of psychosocial function and studies that used the SF-36 or related measures did not show impairment in psychosocial function. It is not known how these data will generalize to community-dwelling, adult MidSoutherners with limb loss.

\section{The Concept of Pain}

This section on the concept of pain will begin by an overview of the history of pain. Pain with limb loss will then be discussed. Pain with limb loss will be organized by the locations of phantom limb pain, residual limb pain, and other pain. 


\section{Introduction to the Concept of Pain}

Until the past few centuries, pain was just viewed as a part of life. ${ }^{72}$ Pain was not viewed as a medical or health concern historically, although today it is a health care issue. Pain historically was tied closely to religious beliefs, with religion and spirituality having an influence on pain perception. ${ }^{73}$ The concept of pain is still studied by theologians, and pain treatment can be a religious and ethical issue. ${ }^{74}$

Pain became the domain of surgeons when general anesthetics were developed and surgeons used anesthetics to perform surgical procedures without pain. ${ }^{72}$ This early form of pain management was specific to the procedures performed and was used more for the advantage of the surgeon and less for the comfort of the patient. As pain treatments progressed to include opiates and analgesics, pain treatment changed. Pain, such as headaches, became a treatable phenomenon, and the specificity model of pain emerged. $^{75}$ The pain specificity model is a cause and effect model where a specific pain stimulus results in a predictable pain experience. This involves one neuron to feel the pain, one neuron to transmit the information, and one neuron to interpret pain. A painful stimulus was assumed to elicit a predictable response.

Pioneers in modern pain medicine found that the specificity model did not explain all pain. With the development of regional anesthesia, pain specialists such as Bonica $^{72}$ and Livingston ${ }^{75}$ found that some patients did not respond in a straightforward manner, consistent with a specificity theory. This resulted in theories by Livingston that included more than one pain mechanism, as well as the development of pain teams as an approach to pain treatment and research with an interdisciplinary team. As pain research evolved, the gate control theory provided an explanation of pain modulation within the central nervous system. ${ }^{76}$ The new approach to pain became viewed as a more complex experience, and modern pain research approaches pain with this paradigm.

The accepted definition of pain by the International Association for the Study of Pain is "an unpleasant sensory and emotional experience associated with actual or potential tissue damage or described in terms of such damage."42 This definition addresses the subjective sensory experience of the suffering that is the common component of all pain. The importance of the personal experience of pain was captured by the nurse McCaffery, who defined pain solely in subjective terms: "Pain is whatever the experiencing person says it is, existing whenever he says it does.",77

The McGill Pain Questionnaire (MPQ) was a landmark in the modern approach to pain assessment, as it was developed to capture the multidimensional pain experience. $^{78}$ It was designed to quantify more than just the presence of pain, but the entire pain experience. The MPQ quantified pain location, pain intensity, pain quality with sensory descriptors, affective descriptors, and temporal pattern descriptors. This captures the pain domains of location, intensity, descriptor, and pattern. These domains are now considered essential to a comprehensive pain assessment. 


\section{Pain by Location}

The following includes a review of literature by pain locations that are common in persons with limb loss. These specific locations represent not only different pain areas but also different etiologies of pain in persons with limb loss. The pain locations of phantom limb, residual limb, and other pain are described below.

\section{Phantom Limb Pain}

Phantom limb pain is pain that is felt as though it is coming from a part of a limb that is missing. ${ }^{35}$ Appendix A has a summary of the history and etiologies of phantom limb pain. Phantom limb pain is common in persons with limb loss, and the prevalence of phantom limb pain was reported to be $72 \%-85 \%{ }^{23,79}$ This included persons with limb loss from the Netherlands, ${ }^{79}$ a mostly white male population from Washington state, ${ }^{23}$ a mostly male civilian and all male United States military sample, ${ }^{25}$ and all male veteran populations. $^{27,80}$

Phantom limb pain was reported to have variability in intensity. Previous studies that reported usual phantom limb pain intensity (as assessed by asking respondents the average pain intensity) was $3.3 \pm 2.0,{ }^{27} 5.1 \pm 2.6,{ }^{23} 5.0 \pm 2.7,{ }^{25}$ and $5.3 \pm 4.9^{25}$ on a numeric $0-10$ scale. Worst phantom limb pain intensity was $5.4 \pm 2.6,{ }^{27} 7.4 \pm 2.6,{ }^{25}$ and $7.7 \pm 4.6,{ }^{25}$ with the least pain intensities being $1.3 \pm 1.3,{ }^{27} 2.4 \pm 2.4,{ }^{25}$ and $2.9 \pm 5.1^{25}$ on a numeric $0-10$ scale. The lower phantom limb pain numbers were reported in a study of 30 male military veterans who were traumatic amputees within the last decade. ${ }^{27}$ These lower phantom limb pain intensities may be reflective of newer innovative techniques to prevent phantom limb pain practiced by military physicians today. The remaining studies on phantom limb pain intensity were older studies that included communitydwelling person with limb loss from a mostly male civilian and all male military population, ${ }^{25}$ and a mostly White male sample from Washington state. ${ }^{23}$

Phantom limb pain quality descriptors are commonly reported in persons with limb loss. The domain of phantom limb pain quality descriptors is important because different descriptions of phantom limb pain have been linked to specific etiologies. ${ }^{81,82}$ Previous studies that assessed specific descriptors reported burning (50\%), ${ }^{83}$ cramping $(50 \%),{ }^{83}$ stabbing $\left(50 \%{ }^{83}, 72 \%{ }^{23}\right)$, shooting $(76 \%),{ }^{23}$ and tiring $(50 \%) .{ }^{83}$ A study that assessed for phantom limb pain quality descriptors by open-ended questioning reported these same descriptors in much lower percentages and were hot $(14 \%-15 \%)$, cramp $(14 \%-15 \%)$, sharp shock/shooting (32\% - 33\%), unusual position (4\%), and squeezing $(13 \%-16 \%){ }^{25}$ The populations that have been studied for pain quality descriptors include persons with limb loss from Canada ${ }^{83}$ and a mostly White male sample from Washington state. ${ }^{23}$ Previous studies report that pattern of phantom limb pain was intermittent or episodic in male United States military veterans ${ }^{27}$ and United States veterans and civilians, ${ }^{25}$ as well as constant in persons with limb loss from Canada. ${ }^{83} \mathrm{~A}$ national survey reported the frequency of positive report of phantom limb pain was never $20.1 \%$, sometimes $58.7 \%$, and always $21.2 \%{ }^{26}$ 
In summary, it is known that phantom limb pain is common. It was not identified how persons with limb loss will rate phantom limb pain intensity using a verbal descriptor scale (VDS) to rate intensity. Verbal descriptors are consistently reported by persons with limb loss to describe the quality of pain. It is known that phantom limb pain intensity changes over time and can be intermittent or continuous or both. The studies that were found on phantom limb pain did not have a large representation of Non-White minorities or women and did not recruit samples from the Mid-Southern United States. It is not known how previous studies about phantom limb pain can be generalized to community-dwelling, adult Mid-Southerners with limb loss.

\section{Residual Limb Pain}

Residual limb pain is pain in the intact part of the limb with an amputation. Residual limb pain is sometime referred to as stump pain. Residual limb pain is also common, although it has been reported to be slightly less common than phantom limb pain, with the prevalence of residual limb pain reported to have a prevalence of $53 \%$ $76 \%$ in persons with limb loss in mostly White male samples from Washington state, ${ }^{23,24}$ from Ireland ${ }^{84,85}$ and the Netherlands. ${ }^{79}$ The pain pattern of residual limb pain in people with limb loss was reported to occur intermittent ${ }^{63}$ and both intermittent and/or continuous in persons with limb loss from Ireland ${ }^{85}$, and from Washington State. ${ }^{23} \mathrm{~A}$ study of the frequency of phantom limb pain through a national survey reported residual limb pain never $32.3 \%$, sometimes $45.4 \%$, and always $22.3 \%$. ${ }^{26}$ Although studies have reported the presence of residual limb pain and residual limb pain pattern, there were no studies identified that reported a comprehensive pain assessment of residual limb pain in persons with limb loss that included both pain intensity and pain quality descriptors.

In summary, it is known that residual limb pain is common, as well as that it can be intermittent or continuous in samples recruited from Europe that were mainly White and male from the United States. However, it is not known how this data on residual limb pain can be generalized to community-dwelling, adult Mid-Southerners with limb loss. There is a key gap in the literature to describe residual limb pain with a comprehensive pain assessment, because it is not known how persons with limb loss rate their residual limb pain intensities or which verbal descriptors they may report to describe residual limb pain.

\section{Other Pain}

Previous studies assessed specifically for back pain in persons with lower extremity limb loss and reported that back pain is common in persons with limb loss in samples of mostly White males from Washington state ${ }^{24,86}$ national survey respondents with upper or lower limb loss ${ }^{26}$ and persons recruited in the Netherlands. ${ }^{87}$ There has also been pain reported in the neck/upper back/contra-lateral shoulders in persons with upper extremity limb loss from the United Kingdom. ${ }^{62}$ In persons with unilateral traumatic lower extremity limb loss, pain in the contra-lateral or sound limb knee secondary to osteoarthritis has also been reported in a sample of all male veterans recruited in Washington state. ${ }^{88}$ Pain in the contra-lateral or sound limb was also 
reported to be common in national survey respondents. ${ }^{26}$ Previous studies on people with limb loss and back pain reported that back pain in people with limb loss could be intermittent or continuous in a mostly White male population from Washington state. ${ }^{86}$ A study of the frequency of phantom limb pain through a national survey reported back pain never $37.7 \%$, sometimes $44.8 \%$, and always $17.5 \%{ }^{26}$ This same study reported that the contra-lateral or sound limb pain frequency was never $50.9 \%$, sometimes $38.6 \%$, and always $10.4 \%{ }^{26}$

Pain in areas other than the phantom or residual limb may have many different etiologies. Over-use injuries were observed more often than what is seen in the general population in persons with upper extremity limb loss from the United Kingdom. ${ }^{62}$ There were different anatomical and physiological measurements found when persons with lower limb loss who had back pain were compared to those who did not, suggesting that back pain in persons with limb loss contributes in a unique way to impaired function. ${ }^{89}$

Six studies identified above evaluated pain in locations other than the phantom or residual limb. These studies evaluated only the specific pain sites of back pain, neck pain, shoulder pain, or contra-lateral sound limb pain. The study of pain only in specified locations other than the phantom limb and residual limb is a narrowly focused approach to describing the pain. In conclusion, previous studies on pain in persons with limb loss have focused on phantom limb pain and residual limb pain, and to a lesser extent on back pain, neck pain, shoulder pain, or contra-lateral sound limb pain. However, persons with limb loss may have pain in other locations. A patient-centered approach to pain assessment in persons with limb loss needs to include all pain locations. This is a key gap in the pain assessment literature of persons with limb loss.

In summary, it is known that it is common for persons with limb loss to have pain in places other than the phantom limb or residual limb and that it can be intermittent or continuous. There was a gap in the literature identified for how persons with limb loss described pain in locations other than the phantom limb or residual limb. It is known that persons with limb loss have back pain and pain in the contra-lateral sound limb, the phantom limb and residual limb. It was not known how community-dwelling, adult Mid-Southerners with limb loss would report pain in locations other than the phantom limb or residual limb.

\section{Number of Pain Locations}

The studies that were identified to have reported on pain reports in more than one location and not just phantom limb pain and residual limb pain are described previously. However, there were no studies identified that assessed for the number of pain locations or the total surface area in pain of persons with limb loss. The number of pain locations of persons with limb loss is unknown. 


\section{The Relationship of Function with Pain, Health Status, and Demographics}

\section{Relationships of Function and Pain}

The following review of literature is organized by the pain locations of phantom limb, residual limb, and other pain.

\section{Relationship of Function and Phantom Limb Pain}

Phantom limb pain predicted variability of global function with the Dutch version of the SF-36 in persons with limb loss in the Netherlands. ${ }^{79}$ Report of phantom limb pain was related to impaired physical function shown by decreased mobility in persons with limb loss from the Netherlands. ${ }^{79,} 90$ The report of phantom limb pain was also related to impaired psychosocial function measured by increased depressed mood and increase in pain being bothersome in national survey of respondents with upper or lower limb loss. ${ }^{26}$ The relationship of report of phantom limb pain and psychosocial function was shown in persons with limb loss from Ireland measured with the Trinity Amputation and Prosthesis experience Scale. ${ }^{85}$ The relationship of report of phantom limb pain and psychosocial function was also demonstrated in a study that showed phantom limb pain precedes stress in male United States veterans. ${ }^{91}$

The relationship between phantom limb pain intensity and psychosocial function has also been demonstrated in the following studies. Increased usual phantom limb pain intensity has been reported to predict impairment in pain-related interference of activities of daily living in a mostly White samples of persons with limb loss living in Washington state and national survey respondents. ${ }^{64}$ Usual phantom limb pain intensity predicted variability of psychosocial function including "bothersomeness" in persons with limb from a mostly White sample from Washington State, ${ }^{23}$ and national survey respondents. ${ }^{26}$ Additionally, increased phantom limb pain intensity was related to greater impairment of psychosocial function documented by an increase in depressive symptoms measured with the Center for Epidemiological Studies depression scale and pain interference as measured by the Pain Interference Scale in persons with limb loss living in Washington State. ${ }^{92}$ Increased phantom limb pain intensity was related to impaired psychosocial function measured by adjustment to limitation in Irish persons with limb loss. ${ }^{85}$

The Bothersome rating of pain is a measure of function that includes the affective influence of pain intensity. This relationship might be inherent in the pain experience. Bothersome is a subjective term of self-appraisal of the influence of pain and captures psychosocial function as well as subjective global function. However, depressive symptoms and adjustment to limitation are more distinct measures of psychosocial function and demonstrate that a unique measure of psychosocial function was impaired by usual phantom limb pain intensity. 
In summary, it is known that any report of phantom limb pain and increased phantom limb pain intensity are related to impaired global function, impaired physical function, and impaired psychosocial function. It is also known that the higher the average phantom limb pain intensity is, the greater the impairment of physical, and psychosocial function. There were no studies identified that reported the relationship of phantom limb pain intensity with function except average phantom limb pain intensity. There were no studies identified that reported how the phantom limb pain quality descriptor or phantom limb pain pattern were related to function. Additionally, no studies compared the relationship of phantom limb pain to function when function was measured with the SIP68. It is not known how reports of phantom limb pain or the studies which report that average phantom limb pain intensity predicts function can be generalized to community-dwelling Mid-Southerners with limb loss.

\section{Relationship of Function and Residual Limb Pain}

Residual limb pain predicted variability of global function with the Dutch version of the SF-36 in persons with limb loss in the Netherlands. ${ }^{79}$ Physical function, specifically observed mobility, was impaired with the presence of residual limb pain in persons with limb loss from the Netherlands. ${ }^{90}$ Increased residual limb pain intensity predicted impaired pain-related disability as measured by the Trinity Amputation and Prosthesis Experience Scales in Irish persons with limb loss. ${ }^{85}$ Worst residual limb pain intensity was reported to be more bothersome than worst pain intensity in the phantom limb or back in persons with limb loss in a mostly White male sample from Washington. ${ }^{24}$ Marshall et al reported that variance of usual residual limb pain intensity was predictive of pain-related impairment, as measured with the Brief Pain Inventory, compared to usual phantom limb pain intensity or usual back pain intensity in persons with limb loss from a mostly White sample from Washington state and national advertisement respondents. ${ }^{64}$ However, Marshall et al report that multiple regression analyses showed that the individual contributions of usual pain intensities of the phantom limb, residual limb pain, and back individually explained no more than $9 \%$ of the variance in pain-related disability, with the combined variance of these pain sites explaining $20 \%$ of the variance of pain-related disability. ${ }^{64}$

In summary, it is known that greater residual limb pain intensity is predictive of the variance of global function, pain-related interference, and increased pain intensity is predictive of an increase in pain being bothersome. It was not known how this will generalize to the relationship between function and residual limb pain in communitydwelling, adult Mid-Southerners with limb loss. There were no studies identified that compared the relationship of residual limb pain quality descriptors or residual limb pain pattern with function.

\section{Relationship of Function and Other Pain}

Previous studies reported that pain intensity in locations other than the phantom limb pain or residual limb pain and pain in the back or contra-lateral sound limb was bothersome in persons with limb loss from a mostly White sample from Washington 
state and national advertisement respondents. ${ }^{23,24,64,86}$ Chronic low back pain intensity was related to impaired physical function as measured with walking speed in persons with unilateral lower extremity limb loss in the United Kingdom. ${ }^{93}$ Back pain intensity in persons with limb loss was shown to have a positive relationship with depressive symptoms in a sample of mostly White national survey respondents with upper $(10.9 \%)$ or lower limb loss. ${ }^{26}$

In summary, it is not known how the greatest pain in areas that are not the phantom or residual limb influences function. It is known that increased back pain intensity predicts an increase in pain being bothersome, and pain in the contra-lateral sound limb is related to impaired physical function. However, since there is a gap in the literature on person-centered pain assessment in persons with limb loss, there is also a gap in knowledge of the influence of pain on function in persons with limb loss who report pain in locations other than the phantom limb or residual limb.

\section{Relationship of Function and Number of Pain Areas}

There were no studies identified that investigated the relationship of the number of pain areas and function. However, Marshall et al showed that if more than one pain site was entered into a multiple regression with the outcome of pain interference as measured with the Brief Pain Inventory, a greater amount of the variability was explained by including more than one pain intensity. ${ }^{64}$ This suggests that the variability of pain from more than one location may explain more variability of pain-related impairment of function.

\section{Relationship of Function and Health Status}

\section{Relationship of Function and Diabetes}

Diabetes is related to impaired global function after an amputation, as measured with the SIP136 in persons with limb loss from the Netherlands. ${ }^{7}$ The result of low global function in persons with limb loss is that persons with limb loss and diabetes frequently enter long-term care and do not return to community life. Additionally, there is a low survival rate after limb loss in persons with diabetes. In-hospital mortality for older United States veterans undergoing amputation for vascular causes was $9.7 \%$ after below-the-knee amputation and 16.5\% after above-the-knee amputation. ${ }^{94}$ The five year survival rate after a lower limb amputation from diabetes in Brazil was reported to be $42.4 \%,{ }^{95}$ and $34.7 \%$ in the Northeastern United States. ${ }^{96}$ Co-morbidities, including diabetes, cardiopulmonary disease, other diseases, and disability have been associated with poorer physical function in people with limb loss from the Netherlands. ${ }^{60}$ In a study of persons with diabetes from Texas, an amputation was related to greater impairment of global function and physical function, but not psychosocial function as measured with the SIP136. ${ }^{6}$ This suggests that diabetes in itself influences psychosocial function that does not change with an amputation. There were no studies identified that 
looked at diabetes as a separate variable from cause of limb loss in describing the relationship between diabetes and function.

To summarize, diabetes is associated with impaired function in persons with limb loss. Persons with diabetes had high mortality, and morbidity rates which may decrease function and the ability to live independently in the community. Among persons with diabetes, although global and physical function changed with amputation, psychosocial function did not. It is not known how this data will generalize to the relationship between diabetes and function in community-dwelling, adult Mid-Southerners with limb loss.

\section{Relationship of Function and Smoking}

Previous studies by Mackenzie et al reported that a history of smoking was related to impaired global function, physical function, and psychosocial function in LEAP study participants SIP136. ${ }^{4,5}$ Mackenzie et al did not differentiate between current or former smokers when they reported their findings and reported smoking history only. There were no studies identified that described the relationship of smoking with function in persons with limb loss from any cause. There is a gap in the literature to describe the influence of smoking on function in persons with limb loss from any cause. It is not known how the relationship between smoking and impaired function of persons with traumatic limb loss will be generalized to community-dwelling, adult MidSoutherners with limb loss.

\section{Relationship of Function and Obesity}

Obesity did not predict mobility or the ability to live independently measured as by chart review in a sample of person with limb loss from South Carolina. ${ }^{97}$ This population was $63.1 \%$ White and $57.8 \%$ male and may share some cultural characteristics with the Mid-South. There were no other studies identified that specifically reported any relationship between obesity and function. There is a gap in the literature to describe the relationship between obesity and global function, physical function, and psychosocial function in persons with limb loss.

\section{Relationship of Function and Self-Assessment of Health}

There is a gap in the literature in self-assessment of health and function in persons with limb loss. A study of older adults in the general population from sites throughout the United States reported no consistent relationship between self-assessment of health and function after a major medical event. ${ }^{98}$ One study reported that perceived health was related to balance in persons with lower extremity limb loss from Canada (male 74\%). ${ }^{99}$ Balance may affect the mobility control category of physical function. Over half of the LEAP study participants with limb loss, despite reports of impaired global function, physical function, and psychosocial function, rated their health as "excellent", which represented the best rating of health. 4, 5, 61 However, it was not identified if this relationship had been explored statistically. 


\section{Relationship of Function and Amputation History}

There is a gap in the literature, as no studies were found that evaluated the relationship of function to the number of limbs lost. However, there are studies that reported a high level of function in persons with more than one limb lost. United States Vietnam veterans with bilateral lower limb loss have been reported to acquire the function level to lead "normal" lives. ${ }^{100}$ There are single case reports of persons with bilateral limb loss achieving a high level of function with the appropriate prosthetic devices. $^{101,102}$

There was only one study found by Demet et al, that compared the relationship between limb loss type, upper versus lower limb loss, with function. ${ }^{103}$ This was a crosssectional study of 539 French veterans as well as self-employed persons with limb loss living in East France. Only $14.7 \%$ of the male sample and $11.3 \%$ of the female sample had upper extremity limb loss only. Demet et al reported that lower extremity amputation was predictive of impaired global function related to physical disability, energy level, and emotional reactions using the Nottingham Health Profile.

Amputation level has been associated with variability of function impairment. Mackenzie et al reported no difference related to amputation level above versus below the knee to measure for global function, physical function, or psychosocial function using the SIP136 in LEAP study participants. ${ }^{4}$ However, they reported that persons with below the knee limb loss had a greater walking speed than persons with above the knee amputation levels. The Frenchay Activities Index, a survey instrument, and mobility scores were not related to amputation level in persons with lower extremity limb loss from Canada. ${ }^{104}$ The SIP136 global function showed greater impairment with more proximal levels of limb loss in persons with diabetes in Texas. ${ }^{6}$ It is known that more proximal lower extremity limb loss is related to increased energy requirements for ambulation, so a more proximal amputation level causes more fatigue with ambulation, which should contribute to impaired mobility. ${ }^{105}$ In summary, the literature is inconclusive on the relationship of amputation level and function. Subjective measures of function may not be related to amputation level because they do not have the sensitivity or specificity to measure how function is influenced by amputation level. All persons with limb loss must achieve a minimum proficiency to ambulate with a prosthetic limb, and traditional function and mobility instruments may not measure the impact of amputation level on physical function, specifically mobility once this level of proficiency is achieved.

Amputation duration was not related to global function in people with limb loss who lost limbs as adults, comparing the total SIP136 score in LEAP study participants, ${ }^{4}$, ${ }^{5}$ but psychosocial scores did show greater impairment over time. ${ }^{5}$ Amputation duration was related to better global function and social behavior when limb loss occurred in childhood in French persons with upper and lower limb loss. ${ }^{103}$ To summarize, it is known that the relationship between amputation duration and function is different when amputation occurs in childhood versus adulthood. The relationship between amputation duration and function may not be straightforward in persons who lose limbs as adults. It 
is not known whether the relationship between amputation duration and function will generalize to community-dwelling, adult Mid-Southerners with limb loss.

The literature on the relationship between amputation cause and function is inconclusive. Amputation cause defined as either vascular or traumatic did not influence function at discharge from in-patient rehabilitation when function was measured by the Functional Independence with limb loss in a sample from the United States. ${ }^{106}$ Since a minimum level of function is required for discharge clinically, the timing of this measure may have prevented any differences in function from being detected. Persons with traumatic limb loss had quicker walking speed and better physical fitness than persons with lower extremity vascular limb loss from California. ${ }^{107}$ Since there are conflicting results in the literature regarding limb loss cause and function, this relationship may be complex. Traumatic limb loss may be associated with co-morbidities associated with major trauma, and vascular limb loss may be associated with vascular co-morbidities. Any difference between function in persons with traumatic or vascular limb loss may be unique to the sample populations. The relationship between limb loss cause and function of community-dwelling, adult Mid-Southerners with limb loss is not known.

Although amputation history has a role in clinical treatment of persons with limb loss, the influence of all specific amputation history variables on function has not been fully explained. There is a gap in the literature regarding the influence on function by the number of limbs lost and amputation type: lower vs. upper extremity. There are inconsistencies in the literature regarding the influence on function by amputation level, amputation duration, and amputation cause.

\section{Relationship of Function and Demographics}

\section{Relationship of Function and Age}

The relationship of increased age with decreased function in persons with limb loss has been well documented and is discussed below. Age was associated with better function, measured with the Toronto Extremity Salvages Score in adult survivors of childhood limb loss (male 48.9\%) from the United States. ${ }^{108}$ However, Molton et al reported that the relationship between age and pain interference, as measured with the Brief Pain Inventory, diminished with increasing age, such that younger people with limb loss had more pain interference than older people with limb loss from Washington State (male $67 \%$, White $81 \%$ ). ${ }^{109}$ Increased age in persons with limb loss was associated with decreased physical function in persons from the Netherlands, ${ }^{7,60,90}$ and persons with upper and lower extremity limb loss from France. ${ }^{103}$ An increase in age in persons with limb loss was specifically associated with impaired mobility and balance in persons from Canada as measured with using the Prosthetic Evaluation Questionnaire mobility scale, ${ }^{99,110}$ impaired mobility in persons with limb loss from Florida, ${ }^{111}$ and persons with limb loss from South Carolina. ${ }^{112}$

It is known that increased age is associated with impaired function in persons with limb loss, and more specifically mobility. However, with age there are increased 
coping skills with regard to pain interference. It is not known whether the relationship of age with impaired function will generalize to community-dwelling, adult MidSoutherners with limb loss.

\section{Relationship of Function and Gender}

The literature is equivocal regarding the relationship of gender with function. Male gender of persons with limb loss was associated with less global function impairment in French persons with upper or lower limb loss as measured by the Nottingham Health Profile, ${ }^{103}$ persons from Canada as measured with using the Prosthetic Evaluation Questionnaire, ${ }^{99,110}$ and the Toronto Extremity Salvages Score in adult survivors of childhood limb loss (male 48.9\%) from the United States. ${ }^{108}$ Male gender was associated with greater physical function impairment measured by the subscores of the SIP136 in LEAP study participants at seven years amputation duration, ${ }^{5}$ although no difference was found at two years amputation duration. ${ }^{10}$ Male gender was related to less physical impairment, as measured with the physical disability scale of the Nottingham Health Profile. ${ }^{103}$ Additionally, male gender was associated with less physical function impairment overall in persons with limb loss from Canada measured by balance, which is indicative of mobility. ${ }^{99}$ In contrast to these data, male gender was related to greater physical impairment as measured with observed walking speed with LEAP study participants. ${ }^{4}$ Gender was not related to psychosocial function measured with the psychosocial function sub-scales of the SIP136 persons with limb loss from Washington state ${ }^{92}$ or LEAP study participants. ${ }^{4,5}$ However, male gender was associated with better psychosocial function in French persons with upper or lower limb loss as measured with Emotional Reaction scale of the Nottingham Health Profile. ${ }^{103}$

To summarize, the studies of gender and global function, physical function, and psychosocial function are unequivocal. Male gender was associated with better physical function measured with surveys, contrasted by more physical function impairment measured with observed mobility. Gender was associated with no difference in psychosocial function with the SIP136 but was associated with less impairment with a different survey. The relationship between function and gender in community-dwelling, adult Mid-Southerners with limb loss is not known.

\section{Relationship of Function and Race/Ethnicity}

Studies have reported that Non-White race/ethnicity is related to impaired global function, physical function, and psychosocial function as evidenced by SIP136 scores in persons with limb loss from the LEAP study. ${ }^{4,5}$ No other studies were discovered that evaluated the relationship of function with Non-White race/ethnicity. There is a gap in the literature to evaluate the influence of race/ethnicity on function in persons with limb loss. It is not known whether the relationship of global function, physical function, and psychosocial function and Non-White race/ethnicity will generalize to communitydwelling, adult Mid-Southerners with limb loss. 


\section{Relationship of Function and Education}

Education less than college level was associated with impaired global function, impaired physical function, and impaired psychosocial function, as evidenced by SIP 136 ${ }_{5}$ scores in persons with limb loss from the Lower Extremity Assessment Project LEAP. ${ }^{4}$, ${ }^{5}$ No other studies were found that evaluated the relationship of function and education in persons with limb loss. There is a gap in the literature to evaluate the relationship of function with education in persons with limb loss. It is not known how the relationship of impaired function in persons with less education will generalize to communitydwelling, adult Mid-Southerners with limb loss. 


\section{CHAPTER 3: METHODS}

The study methods are presented in this chapter, including a detailed description of the study methods and the psychometrics of the instruments. The analysis section is organized by research question.

\section{Study Design}

This study was a descriptive cross-sectional survey using an intervieweradministered structured questionnaire with persons with limb loss living in the MidSouth area. Participants were interviewed once, and the interviews were conducted either face-to-face or over the telephone. Surveys were administered by an interviewer, because it was anticipated that some participants might have low literacy skills. ${ }^{113}$ Participants had the option of being interviewed in-person or over the telephone to achieve a sample throughout the Mid-South. It was assumed that many persons with limb loss may have limited mobility, which increases the burden of meeting outside their homes. The study was approved by the University of Tennessee Health Science Center Institutional Review Board (IRB). A copy of the approval letter is located in Appendix B.

\section{Site}

Participants were interviewed at a location of their choosing in the Mid-South. The Mid-South states are Alabama, Arkansas, Kentucky, Mississippi, and Tennessee. In person, face-to-face interviews were conducted at a public meeting place of the participant's choosing and could include support group meeting facilities and limb loss service provider's offices. The rationale for choosing these locations was to provide convenience to the participants and to reach persons who would otherwise not participate in surveys. Telephone interviews originated from the participant's choice of location to include persons from throughout the wide geographic area of the Mid-South. Persons could be interviewed by phone, if they chose.

\section{Sample}

Inclusion criteria included 1) community-dwelling persons; 2) limb loss at a higher level than a finger or toe; 3) residency in the Mid-South area; 4) age 18 years or older; 5) at least six months post-operation from the last amputation or revision; 6) ability to understand spoken English; and 7) ability to communicate verbally. The rational for these sample characteristics is discussed below.

Only community-dwelling persons were included because it was anticipated that the population would be more homogenous with regards to function. Additionally, the 
number of persons with limb loss, living in the community with pain was under reported by primary care clinicians. ${ }^{28}$ Persons with limb loss who live in the community may have pain that has not been assessed by and/or reported to a health care provider. This assumption was made because it was reported that persons with limb loss are reluctant to discuss the issue of phantom limb pain with their health care provider. ${ }^{114}$ Therefore, only community-dwelling persons with limb loss were included.

Persons with limb loss greater than a toe or finger were included. It has been demonstrated that a more proximal level of amputation was associated with a lower level of function. ${ }^{4,6}$ Additionally, other studies have used this definition of limb loss. ${ }^{112,115}$ Therefore, having an existing amputation that was greater than a finger or toe was the amputation level criterion to be a study participant.

Participant recruitment was limited to persons with limb loss living in the MidSouth. The demographics and health status characteristics of Mid-Southerners contribute an increased risk for amputation. The states within the Mid-South share a common culture and lifestyle as well as common resources and access to health care. The Mid-South states have high rates of poverty and poor educational attainment, which are traits that have been under-represented in previous limb loss studies of pain and function. Therefore, only persons with limb loss living in the Mid-South were included in this study.

Participants needed to be at least 18 years of age or older, but there was no upper age limit. The age-related domain that defines function as assessed by activities of daily living for children with disabilities is different than for adults with disabilities. ${ }^{116}$ Function domains change during the transition between childhood and adulthood. Therefore, only persons over 18 years were included.

Participants needed to be at minimum of six months post-operation since their last amputation or revision. There is a precedence for six months after an amputation to measure function in pain in persons with limb loss. ${ }^{117}$ After a six month recovery period, persons with limb loss should not have the anticipated acute post-surgical pain. ${ }^{118}$ Pain that is experienced for greater than four to six months is usually considered chronic pain and therefore not acute post-surgical pain. ${ }^{119}$ Therefore, after six months acute surgical pain should be resolved, and pain that is experienced is not specifically related to normal surgical healing. Schoppen found that in $69 \%$ of persons undergoing an amputation, the SIP136 administered during the perioperative period was predictive of function at one year post amputation. ${ }^{120}$ This suggests that the level of function for some may plateau early in the post surgical phase. However, by waiting for six months, individuals have a greater chance of being fitted with a prosthetic limb, and they usually have had gait training by this time. Additionally, at six months most persons who are going to participate in inpatient rehabilitation have had the opportunity to do so.

There is a high mortality rate for persons with vascular limb loss. ${ }^{95,96}$ A high mortality rate in persons with limb loss demonstrates that at the time of the amputation, individuals' health has already declined. Since limb loss can be associated with end of 
life, community reintegration may not be fully obtained by all, and describing pain and function in this population after having time to fully recover from the acute pain of surgery can give useful information regarding the need for palliative care.

Persons with limb loss did not have to report pain in any location to participate in this study. The limb loss studies identified and presented in Chapter 2 have all reported that most persons with limb loss report either or both phantom limb pain and/or residual limb pain. The PI assumed that most persons with limb loss have pain. Those persons with limb loss who did not report pain may provide information on how living pain free affects the other variables in the study.

Participants had to be able to understand spoken English. The recruitment of participants able to understand English provided a more homogenous population, thus eliminating threats to internal validity by administering the instrument in more than one language. The consent and instrument (Appendix C and Appendix D) were read out loud to participants, so there was no need for them to have the ability to read. However, comprehension of spoken English was required. Since the Mid-South has the largest percentage of the population with low literacy in the United States, ${ }^{113}$ every survey was administered verbally. This was to accommodate persons of all literacy levels and to provide consistency in data collection procedures.

All participants had to be able to participate in the interview and answer the questions themselves. Function and pain were assessed through self report to capture the subjective component of pain. Therefore, only participants were interviewed; no proxy interviews were used.

\section{Study Instruments}

\section{Measurement of Function}

\section{The Sickness Impact Profile 68}

To assess function, a global self-report instrument was chosen. The SIP68, an abbreviated version of the original SIP136, was used to measure function (Appendix C). The SIP136 will be described, followed by a summary of the development of the SIP68, the psychometrics of the SIP68, and applications of both the SIP136and SIP68 to populations similar to the population of study. Then the modifications and SIP68 scoring, administration, and pilot testing will be discussed.

The SIP136 is a 136 item instrument that was developed to measure sicknessrelated dysfunction. ${ }^{121}$ The pilot testing during the development of the SIP136 included only persons living in the Northwestern United States. The SIP136 measures sicknessrelated dysfunction by self-report. The developers of the SIP136 assumed that function changes that result from an impairment or illness is an individual experience and is not 
always consistent with a diagnosed impairment or illness. Therefore, the SIP136 does not include information from an individual's health history. This is a scaled instrument with the aim of measuring function only for the immediate 24 hour period at the time of administrating the instrument. The SIP136 can detect differences in persons as their sickness-related dysfunction changes.

There are two domains of the SIP136: physical and psychosocial. ${ }^{58,122}$ The physical domains are ambulation, mobility, body care, and movement. The psychosocial domains are sleep and rest, eating, work, home management, recreation and pastimes, social interaction, alertness behavior, emotional behavior, and communication. These domains and categories were developed by consulting with experts. There were no statistical tests to show that items in each category showed convergent validity, as some categories had only a few items, which would make the statistical analyses meaningless. Convergent, discriminate, and clinical validity of the SIP136 was demonstrated in the final stages of development. ${ }^{123}$

Although the SIP136 could be administered in about half an hour, it contained 136 statements, and for some persons this was too long. The SIP68 is a 68 item instrument developed in the Netherlands in the 1990s through secondary analyses of published studies with 10 different diagnostic groups that included 2,527 subjects who had been administered the Dutch version of the SIP136. ${ }^{67}$ The aim of this study was to develop a shorter instrument and determine if the a priori categorical structure was evident through statistical evaluation. The statistical methods used included a principle components analyses (PCA). The PCA is an internal testing of the convergent validity. After developing the SIP68, the results were compared to the original SIP136.

Before analyzing all of the items from the SIP136 to develop the SIP68, de Bruin et al removed the category of work, as there was missing data since many persons did not work before their illness. ${ }^{67}$ Items that were highly skewed, with a $90 \%$ or greater agreement of either positive or negative responses in all subjects within a diagnostic group, were also removed because they lacked sensitivity. A regression analyses was performed to determine if the total score on the SIP68 was consistent with the total score on the original SIP136. The correlations of the SIP68 were compared to the SIP136 total score and the physical and psychosocial domains showed a strong correlation of at least .94 .

The results of the PCA analyses did not support the 12 categories of the SIP136. ${ }^{67}$ A PCA identified a six-factor model. A series of PCAs was performed, and items that had a loading of less than 0.40 for a factor were eliminated. The series of PCAs was halted when it appeared that the standard items in the areas of communication and eating might be eliminated, and the new instrument had 68 of the original 136 items. The authors stopped eliminating items here, as they felt it was the best compromise for shortening the instrument and maintaining validity. The six new categories that emerged empirically and statistically were somatic autonomy (SA) with 17 items, mobility control (MC) with 12 items, psychic autonomy and communication (PAC) with 11 items, social behavior (SB) with 12 items, emotional stability (ES) with 6 items, and mobility range 
(MR) with 10 items. The total score for the SIP68 of all 68 items represents global function. De Bruin et al acknowledged that a limitation of the development of the SIP68 was that content validity could not be directly compared between the SIP68 and the SIP136 because different and fewer categories emerged statistically from the empirical data.

Criterion validity and test-retest reliability of the SIP68 was assessed when the SIP68 was administered to a sample of persons with lower extremity limb loss from the Netherlands that also were administered the Prosthetic Profile of the Amputee (PPA). ${ }^{3}$ The PPA was developed specifically for persons with lower extremity limb loss to assess for prosthetic limb use and activities. ${ }^{124}$ A direct statistical analysis to compare all of the specific results of the instruments was not entirely practical since the PPA has many qualitative questions. However, both measures remained stable, with $85-90 \%$ of the study participants showing that both measures of function remained stable. This magnitude of stability suggests that the SIP68 has criterion validity and test-retest reliability with lower extremity persons with limb loss.

Criterion validity of the SIP68 was also shown by comparing the SIP68 to the Nottingham Health Profile in persons that were wheel chair dependent. ${ }^{67}$ Spearman correlations were used to compare similar categories. Spearman's $r$ comparing the Nottingham Health Profile's physical mobility and the SIP68's somatic activity was 0.68. Spearman's $r$ comparing the Nottingham Health Profile's emotional reactions and the SIP68's emotional stability was 0.56 . The magnitude of these correlations suggests that the SIP68 has criterion validity for somatic activity and emotional stability in persons who are wheel chair dependent. Schoppen et al administered the SIP68 to older adult Dutch persons with dysvascular unilateral lower limb loss from two weeks to one year post amputation and reported criterion validity by comparing the SIP 68 to observational measures. ${ }^{60}$

Since de Bruin et al developed the SIP68 categories statistically, Cronbach's alphas for each category were statistically significant. ${ }^{125}$ These were reported as the following Cronbach's alphas: $\mathrm{SA}=0.78 ; \mathrm{MC}=0.85 ; \mathrm{ES}=0.72 ; \mathrm{SB}=0.81 ; \mathrm{MR}=0.79$; and $\mathrm{PAC}=0.77$. The convergent validity of the SIP68 in wheel chair users was demonstrated by Post el al. who reported the following Cronbach's alphas for each of the SIP68 categories: $\mathrm{SA}=0.85 ; \mathrm{MC}=0.53 ; \mathrm{ES}=0.67 ; \mathrm{SB}=0.79 ; \mathrm{MR}=0.78$; and $\mathrm{PAC}=$ $0.7{ }^{126}$ These results demonstrated reliability to use the SIP68 with a population of wheel chair users. However, the lowest measure of reliability was with the mobility control scale. This may be due to the significant variations in the level of mobility control among wheel chair users that are not as well correlated within this category as an outcome variable compared to the other categories.

During the development of the SIP68, the test-retest correlations of the test administered by different methods were 0.80 to $0.88 .{ }^{122}$ When persons with limb loss were administered the SIP68 two months apart, there was no significant change. ${ }^{3}$

However, when the SIP68 was administered at status post amputation two weeks and six weeks, there was a small difference in the scores ${ }^{60}$ In a study of United States residents 
with disabilities, all of the scores within the SIP136 and SIP68 showed a high test-retest reliability, with intra-class correlations ranging from 0.61 to $0.90 .^{127}$

The original work establishing the validity and reliability of the SIP68 was performed in the Netherlands. ${ }^{60,67,126,128}$ Nanda et al performed a study on adults with disabilities to establish validity, retest and proxy reliability, and scaling properties of the SIP68 in the United States population. ${ }^{127}$ The SIP136 was administered by phone for $92 \%$ of 398 participants. The study included the SIP136, the SIP68, Activities of Daily Living Scale, Independent Activities of Daily Living Scale, and the Short Form 36. Test-retest reliability was assessed by administration of the SIP68 twice, with a one week interval, in spinal cord injured veterans. Statistical analyses were performed, with the results of the SIP136 described by de Bruin in the original development of the SIP68. ${ }^{67}$ Categories consistent with the original development of the SIP68 included $\mathrm{PAC}, \mathrm{SB}$, and ES. The items comprising SA displayed two factors, and the items comprising both MC and MR displayed three factors. The results of the total score of the SIP68 were significantly related to all of the categories at the 0.01 level of the Activities of Daily Living Scale, Independent Activities of Daily Living Scale, and the Short Form 36, with correlations that ranged from 0.32-0.59.

Cano et al compared the SIP136 scores of Black and White participants with chronic pain living in the community. ${ }^{129}$ The Physical and Psychological domains of the SIP136 scores were higher in Blacks, which reflects a lower level of function. The kappas for the inter-item reliabilities for the Physical domains were for Blacks $\alpha=0.86$, for Whites $\alpha=0.82$. The kappas for the inter-item reliabilities for the Psychological domains were for Blacks $\alpha=0.91$; for Whites $\alpha=0.87$.

To summarize the psychometric studies of the SIP68, the SIP68 is an abbreviated version of the valid and reliable SIP136, a measure of sickness-related dysfunction. Criterion validity, test-retest reliability, proxy reliability, and internal consistency have been established for the SIP68 specifically. The psychometrics of this instrument are well established. This is an appropriate instrument to measure in community-dwelling, adult Mid-Southerners with limb loss.

The SIP68 requires either a positive response or a negative response. Negative responses were coded as 0 , and positive responses were coded as 1 . A higher SIP68 score was assumed to be a greater level of dysfunction. The scores for each SIP68 category were calculated. A global function score (SIP68 Total) consisted of the scores for all 68 items.

Most Mid-Southerners share a common Southern English dialect. ${ }^{130}$ To ensure that the SIP68 would be appropriate for use with Mid-Southerners, the PI developed prompts to use for the SIP68 items with a consultant. The consultant, who was a professional care assistant who has lived in the Mid-South for over 40 years and had provided direct patient care to both ambulatory and home bound clients for over 20 years, provided guidance in the following. The consultant and the PI reviewed each item individually. The consultant made recommendations on the wording of SIP68 items that 
would be better understood by Mid-Southerners. The PI then reviewed these suggestions with a researcher with experience working with the Mid-South population. These changes were incorporated into the SIP68 to use as prompts if the study participants did not understand the SIP68 item. The prompts were then reviewed verbally with the cultural consultant. The SIP68, with the Mid-South prompts, was pilot tested on community-dwelling White ( 3 females) and Black ( 2 males, 1 female) MidSouth residents, who were then asked if they were able to understand the questions. The pilot participants did not use prompts for every item SIP68 item. However, the pilot participants reported that the prompts helped with understanding the SIP68 items. For this study, the SIP68 was read to participants verbally by the PI. If the participant did not understand the SIP68 item, the prompt was read to the participant.

\section{Limb Loss Impact 12}

Limb loss may contribute to overall function in a unique manner. A separate measure, the Limb Loss Impact Profile 12 (LLIP12), was used to determine if overall health-related function is different from limb-loss related function (Appendix C). The LLIP12 consisted of 12 questions selected from the SIP68, with two items from each category. The LLIP12 was developed by the PI in collaboration with researchers who have a combined expertise in gerontology, function, pain assessment in MidSoutherners, and phantom limb pain. Two items from each category of the SIP68 were chosen for a global representation of the specific impact of limb loss. The directions for the instrument also instructed the participant to answer the questions regarding changes in health related to limb loss but not to overall changes in health function that could be impacted by other factors. The prompts developed for the Mid-Southerners for the SIP68 were used in the LLIP12.

The LLIP12 was coded and scored identical to the SIP68. The LLIP12 required a response to items that were affected by limb loss. Negative responses were coded as 0 , and positive responses were coded as 1. A higher LLIP12 score was assumed to be a greater the level of dysfunction specifically attributed to limb loss. A global function attributed specifically to limb loss score (LLIP12 Total) consisted of the scores for all 12 items.

\section{Measurement of Pain}

Pain is a construct composed of both physical and behavioral domains. Pain is a unique and personal experience that can't be measured by direct observation alone. ${ }^{119}$ Pain has a subjective component that influences pain interpretation and suffering. All pain assessments performed were self-declarations of the individual's pain experience because of the personal and subjective nature of the experience of being in pain. The following pain domains were assessed: location, pain intensity pattern, pain quality descriptor, pain temporal pattern, pain duration, pain treatment, and pain aggravating and alleviating factors. The instrument used to assess of all the specific domains will be discussed below. 


\section{Pain Location}

Pain location was assessed by the pain map from the McGill Pain

Questionnaire. $^{78}$ The pain map is an outline of the front and back of the body. This was modified by having the participant shade the area where pain is felt - a technique described by Sherman and used in research studies with persons with limb loss. ${ }^{131}$ Participants interviewed in person were given a fine tip marker and asked to: 1) draw a line at the exact location of their amputation or amputations and 2) shade in all areas where they had pain. The instructions directed participants to include all areas where pain is experienced in both existing and absent body locations. Participants interviewed by telephone or who were unable to draw were asked to 1) describe the exact location(s) of pain and 2) describe all of the areas where they had pain. These area were recorded on the pain map by the PI. The total number of areas of pain was obtained by analyzing the MPQ map, as described by Escalante et al, ${ }^{132}$ This analyses involves placing a grid with 36 areas over the MPQ pain map to record the specific areas in pain. This grid was developed to identify joint pain in older adults, and was chosen because it provides a detailed analysis of pain in the extremities. The number of pain sites where the individual had pain was totaled. This total is referred to as total number of areas in pain. Pain location was further assessed by asking about specifically if the participant had any pain in the phantom limb, residual limb, or pain in any other area. This was scored as yes or no. Each location was coded $0=$ no, $1=$ yes.

\section{Pain Intensity}

Pain intensity was measured with a verbal descriptor scale. A verbal descriptor scale (VDS) is a pain scale that uses descriptive adjectives to describe pain quantity or level. ${ }^{119}$ The verbal descriptors were no pain, small pain, medium pain, large pain, and pain as bad as it could be. These terms were understood by and valid for a sample of African American and White Mid-South nursing home residents. ${ }^{133}$ The responses were re-coded for analyses using a five point numeric scale with $0=$ no pain, $1=$ small pain, 2 $=$ medium pain, $3=$ large pain, and $4=$ pain as bad as it can be. A VDS scale was preferred among older adults with and without cognitive impairment, ${ }^{134}$ and was understood by African Americans living in the Southern United States. ${ }^{135}$ The anchor words for the VDS were "no pain" at the low end of the scale and "pain as bad as it can be" for the highest pain intensity rating. Many pain intensity scales use the words "pain so bad that you can't bear it for another moment." This was not used, since the term "bear" has religious connection and Mid-Southerners have a high level of religiosity and may associate it with the Christian belief of "a cross to bear."

Pain intensity was assessed for the following: a) pain intensity now, b) usual pain intensity, c) worst pain intensity, and d) least pain intensity. Pain intensity was measured at the phantom $\operatorname{limb}(\mathrm{s})$, residual limb, and the worst other pain area. If a person had multiple limb loss, the pain intensity at the most painful phantom limb and residual limb was used for analyses. The areas with the greatest pain intensity were used, as it was assumed that highest pain intensity would be of greater impact to the individual than an area of lesser pain intensity. There is precedent for this, because Ephraim et al in 
a study of phantom limb, residual limb, and back pain used the most painful phantom and residual limb when there were more than one affected limbs in a single study participant. $^{26}$

\section{Pain Quality Descriptors}

Pain quality descriptors describe the unique sensation or feeling of the pain experience. ${ }^{13}$ This pain quality data was gathered for the following areas with a positive report of pain: the missing limb, the phantom limb, and the other pain with the greatest intensity.

The McGill Pain Questionnaire (MPQ) is a gold standard pain assessment instrument that contains 84 pain quality descriptors in four major groups (sensory, affective, evaluative, and miscellaneous), with 20 subclasses. $^{78}$ A short form of the MPQ was developed with 15 pain quality descriptors from the sensory and affective categories. ${ }^{136}$ Common phantom limb pain quality descriptors have include hot/burning, ${ }^{25,83}$ cramping $^{25,83}$ stabbing, ${ }^{23,83}$ shocking/shooting, ${ }^{23,25}$ tiring, ${ }^{83}$ squeezing or tight band around the arm or leg, ${ }^{25}$ and abnormal position. ${ }^{25}$ All of the above descriptors and twisting, which is similar to abnormal position, were chosen because they have been documented in persons with phantom limb pain. All are included in the MPQ short form except tight band, twisting and abnormal position. The MPQ quality descriptors burning, cramping, sharp, and tiring were shown to be valid for Black and White Mid-South nursing home residents. ${ }^{133}$ Each descriptor was assessed for a positive or negative response for the locations of the phantom limb, residual limb, and other pain. Each item was coded $0=$ no, $1=$ yes.

\section{Pain Pattern}

Pain pattern is the temporal pattern of pain. Pain pattern measurement included continuous pain and intermittent pain. Pain pattern descriptors of the pain are described in the MPQ ${ }^{78}$ but not in the MPQ short form. ${ }^{136}$ The MPQ pain pattern descriptors of intermittent and continuous were chosen, as these represent very distinct temporal patterns. The terms intermittent and continuous were changed to be more understandable by the cultural consultant. Intermittent pain was assessed by asking participants if their pain would come and go. Continuous pain was assessed by asking the participants if they had pain all the time. Each pain pattern was assessed for a positive or negative response for the locations of the phantom limb, residual limb, and other pain. Intermittent pain was coded as $0=$ no, $1=$ yes. Continuous pain was coded as $0=$ no, $1=$ yes. 


\section{Measurement of Health Status}

\section{Diabetes}

A diagnosis of diabetes was determined by self report. Diabetes was assessed by asking participants if they had sugar diabetes, a common term for diabetes in the MidSouth. This item was coded as $0=$ no, $1=$ yes.

\section{Smoking}

Smoking in function and limb loss studies was reported as either past or currently smoking. ${ }^{5}$ Participants were asked about their smoking history now and in the past. Smoking now was assessed by asking participants if they smoke now and was coded as 0 $=$ no, $1=$ yes. For ever smoked, the participants were then asked if they ever smoked; this was coded as $0=$ no, $1=$ yes.

\section{Self Assessment of Health}

Self assessment of health (SAH) is a global rating of one's own health. ${ }^{137}$ Self assessment of health was assessed by single item question, "How would you rate your health?" A verbal descriptor scale was used for self assessment of health. The verbal descriptors for self assessment of health were bad, poor, fair, good, and excellent. Prompts used the terms small, medium, large, with the anchor for the best rating of health as good as health can be, similar to the verbal descriptor scale used to assess for pain intensity. The prompts and scoring for each were $0=$ bad (bad health only), $1=$ poor (small amount of health), 2 = fair (medium amount of health), $3=$ good (large amount of health), and $4=$ excellent (as good as health can be).

\section{Obesity}

Height and weight were assessed by self-report, and no adjustments were made for missing body parts. For lower limb amputees with multiple limb loss, the height wearing a prosthetic limb or before amputation (for persons who did not wear a prosthetic limb) was used. Body mass index (BMI) was calculated using the formula height in inches divided by weight in pounds multiplied by 703 , with overweight defined as a BMI of between 25 and 29.9 and obese was a BMI or 30.0 or greater. ${ }^{138}$ Garrow and Webster reported that this formula estimated body fat with a correlation of 0.943 for men and 0.955 for women. ${ }^{139}$ 


\section{Amputation History}

Amputation health history was a general overview of health status specific to the participants' limb loss. To determine the number of limbs lost, participants were asked how many limbs they lost, and recorded as the exact number of limbs lost. The following four amputation history variables were recorded for each lost limb. Amputation type: lower extremity was coded as $0=$ upper extremity and $1=$ lower extremity. Amputation level: AEA/AKA was determined by if participants had lost at least a major limb articulation (elbow or knee). This was coded as 0 if all knees and elbows were intact, and 1 if there was the loss of at all or part of least one elbow or knee 1. There was precedent to measure amputation level: AEA/AKA in persons with lower extremity amputation as either: above the knee, through the knee, and below the knee ${ }^{4,97}$ or only above or below the knee ${ }^{64,140}$ in previous studies of function and limb loss. There were no studies found that described amputation level as a variable in upper extremity limb loss and function. Amputation duration was recorded in months, with the time since the most recent amputation used in the analyses. Limb loss cause was recorded verbatim. This was re-coded as $0=$ non-traumatic limb loss and $1=$ traumatic limb loss. There was a precedent to distinguish between vascular and traumatic limb loss in studies of function in persons with limb loss. ${ }^{9,106}$

\section{Demographics}

To measure demographics, participants were verbally asked to report on the four items: Age was measured by self report of years. Gender was assessed by self-report as male or female. Gender was coded as $0=$ female, $1=$ male. Race/ Ethnicity was assessed by asking how the participant describes their race or ethnicity. Race/Ethnicity was categorized as $1=$ White, $2=$ Black, $3=$ Latino, $4=$ Asian, $5=$ Native American, and $6=$ other or mixed. This data was re-coded for analyses to $0=$ White and $1=$ NonWhite. Education was recorded as self-report of the number of years of school completed. The percentage of persons that did not graduate from high school was calculated.

\section{Procedure}

This section describes procedures for recruiting participants, ensuring interviewing proficiency, and administration of the questionnaire. Participants were sent a thank you and a summary of results after the study was completed. Details of the procedure are in the following paragraphs.

\section{Recruiting}

A purposive, convenience snowball sampling technique was used to recruit participants from the Mid-South. Persons with limb loss were recruited through 
contacting key persons with the following types of organizations: limb loss support groups, national limb loss advocacy groups, and limb loss service providers.

Entré to the limb loss population began with the PI speaking about phantom limb loss and other limb loss issues with the limb loss support groups. This built a familiar rapport to a core base of persons with limb loss who participated in the following limb loss support groups: a) Out on a Limb (OOAL) in Memphis, TN; b) HealthSouth Amputee Support Group in Memphis, TN, and c) American Amputee Foundation's support group in North Little Rock, AR. Attendees at limb loss support group meetings were offered verbal and written information about the study by the PI or the support group coordinator. The PI also served as a professional advisor to OOAL limb loss support group in Memphis, TN. Attending limb loss support groups meetings and the Amputee Coalition of America's national meeting, the PI was able to network with limb loss service providers, and national advocacy and support groups. National advocacy and support groups that assisted to recruit study participants included Amputee Coalition of America, UpperEx, and Christian Amputee Support Team.

Letters were sent directly to persons with limb loss through traditional mail and e-mailings. Posters and handouts were placed in predominant locations at limb loss service providers throughout the Mid-South. National advocacy groups provided study information on their websites as well as in e-journals. Emails with information about the study were sent to health care providers and limb loss service providers who provided limb loss clients. Participants contacted the PI directly to keep study participation confidential. The specific recruiting roles of each recruiting site are detailed in Appendix E.

Participants expressed interest in the study by contacting the PI by phone, mail, or email. Participants were contacted by phone by the PI to set a meeting time and place or the telephone option with a call time which was mutually agreed upon at that time. The PI gained expertise in using the questionnaire through ten practice interviews with persons from the Mid-South including two Black females, four White females, two Black males, one Asian male, and two White males from the Mid-South.

\section{Power Analyses}

A power analysis using Cohen as a reference was performed to estimate the optimal minimum number of participants needed for multiple regression. ${ }^{141}$ Statistical power of 0.8 or more, alpha of at least 0.05 for a non-directional test, and a moderate effect size as defined by Cohen were assumed. To establish if a relationship between two variables existed using Pearson's r, a sample size of 84 was an optimal minimum number of participants. 


\section{Administration of Survey}

The following is a detailed description of the interview procedure. All interviews were conducted by the PI. Face-to-face interviews occurred at limb loss service provider offices or at limb loss support group meeting places. Participants who were unable to participate in person participated in a telephone interview with the PI. The participant and the PI agreed to a phone meeting date and time. To begin the interview, the PI introduced herself to the participant and provided a verbal, detailed description of the research study. The interviewer read the University of Tennessee Health Science Center IRB-approved consent to the participant, and verbal consent was obtained before beginning the interview. No written consent was required by the University of Tennessee Health Science Center IRB because participation in a verbal survey is considered minimal risk, with no procedures for which written consent outside of the research context would be required. ${ }^{142}$ The telephone interview was conducted identically to the face-to-face interview, with the exception of administering the McGill Pain Questionnaire pain map. The participant would describe where they had pain and then the PI filled out the pain map. The telephone interview pain location assessment then guided the remaining domains of pain assessment. Participants could stop the interview at any time and take a break or drop out of the study. If participants needed to take a break but wanted to continue, the interview was completed within the same day. The survey questions were ordered as follows: demographics, health status, the SIP68, the LLIP12, amputation history, and pain assessment. All participants were sent a thank you letter for their participation at the end of the study.

\section{Analyses}

The analyses section is organized by research question below. The statistical software used for these analyses was Statistical Package for the Social Sciences (SPSS) 17.0. The preset alpha level for significance was 0.05. An alpha between 0.05 and 0.10 was assumed to represent a trend. Data were entered on an SPSS spreadsheet as soon as possible after obtaining the data. All data were proof read after entry to ensure accuracy.

\section{Specific Aim 1}

Specific aim 1 described the function, pain, health status, and demographics of community-dwelling, adult Mid-Southerners. Means, standard deviations, and range were calculated for all continuous variables. Percent was calculated for all dichotomous variables. 


\section{Specific Aim 2}

Specific aim 2 estimated the relationship between function and pain, health status, and demographics in community-dwelling, adult Mid-Southerners with limb loss. Pearson's $r$ correlation was performed to estimate the bivariate relationships of function with pain, health status, and demographics. Function variables were the sum of all items for the SIP68 total, LLIP12 total, and each category of the SIP68 (SA, MC, MR, PAC, $\mathrm{SB}$, and $\mathrm{ES})$.

\section{Specific Aim 3}

Specific aim 3 estimated the joint influences on function by pain, health status, and demographics of community-dwelling, adult Mid-Southerners with limb loss. Multiple regression equations were developed for three function variables: a) SIP68 total to represent the global function domain, b) MR to represent the physical domain, and c) SB to represent the psychosocial function domain. A multi-step process was used to select the candidate predictor variables for pain, health status, and demographics. The process for selecting the three function dependent variables and the candidate pain, health status, and demographic independent variables will be described below.

\section{Selection of Candidates for Dependent Variables}

One function variable was chosen to represent each of three function domains: global function, physical function, and psychosocial function. The SIP68 total was significantly correlated with the LLIP12 total. SIP68 total was chosen to represent the global function domain because SIP68 total has established psychometric properties and was used in studies of function in persons with limb loss.

Variables representing the physical function domain of the SIP68 were SA, MC, and MR. The variable SA was eliminated because of the skewed distribution. The variable MR was chosen because it had larger correlations with the health status variable amputation type: lower extremity and was correlated significantly with the candidate demographic variable Non-White Race/Ethnicity, while SIP68 MC was not. The correlation with Non-White Race/Ethnicity was important because Non-White persons with limb loss have been underrepresented.

Variables representing the psychosocial function domain of the SIP68 were PAC, $\mathrm{SB}$, and ES. The variable PAC was eliminated because of the skewed distribution. The variable $\mathrm{SB}$ was chosen to represent the psychosocial function domain because it was correlated with the demographic variable Non-White Race/Ethnicity, whereas ES was not correlated with any health status or demographic variable. 


\section{Selection of Candidates for Independent Variables}

The number of candidate pain, health status, and demographic variables were reduced using a multi-step process. The process to delineate candidate variables to be a proxy for variables to represent the concepts of, pain, health status, and demographic variables is outline below. First, candidate pain, health status, and demographic variable that were $(p \leq .1)$ correlated with SIP68 total were selected for further evaluation. All residual limb pain variables were eliminated because positive report of RLP was not correlated $(p \leq .1)$ with SIP68. To further reduce the number of candidate variables, a correlation matrix was used to assess for collinearity among the remaining candidate variables. Next the number of candidate variables was reduced by examining the conceptual contribution of each remaining candidate variable to predicting the three function variables. Finally, the list of candidate variables was reduced by considering the statistical relationship with SIP68. The final candidate pain, health status, and demographic variables to be entered into the three regression models for SIP68, MR, and SB were as follows: 1) Usual PLP; 2) Tiring PLP; 3) Tight Band PLP; 4) Intermittent PLP; 5) Continuous PLP; 6) Usual OP; 7) Abnormal Position OP, 8) Intermittent OP, 9) Amputation Type: Lower Extremity, and 10) Non-White race/ethnicity. 


\section{CHAPTER 4: RESULTS}

This chapter will report the results of the statistical analyses. The sample characteristics and internal consistency of function variables will be presented first followed by the results of the three specific aims. The PI interviewed 61 participants. Two participants were unable to complete the pain questions due to fatigue and concern over cell phone minutes. Their data is included in the analyses with the exception of missing pain variables.

\section{Sample}

The demographics of the sample include age, male gender, Non-White race/ethnicity, and education. Demographic information on the study sample is located in Table 4.1.

\section{Internal Consistency of Function Variables}

A Cronbach's alpha was used to assess the internal consistency of the measures of global function (SIP68 total and LLIP12 total), physical function (SA, MC, and MR), and psychosocial function (PAC, SB, and ES). These scores are reported in Table 4.2.

\section{Specific Aim 1}

Specific aim one was to describe the function, pain, health status, and demographics of community-dwelling, adult Mid-Southerners. The results are summarized below.

\section{Function}

The mean scores, standard deviation, and range of the SIP68 total, the LLIP12 total, and the individual categories of SIP68 are listed in Table 4.3.

\section{Pain}

Positive report of pain and the means of pain intensity, pain quality descriptor percentages, and pain pattern percentages are listed by the locations of phantom limb pain (PLP), residual limb pain (RLP), and the worst other pain (OP) (Table 4.4). The locations of other worst pain are listed by type and level of amputation (Table 4.5). 
Table 4.1 Demographic Descriptive Data $(\mathrm{N}=61)$

\begin{tabular}{lc}
\hline \multicolumn{1}{c}{ Variable } & Mean \pm SD (Range) or Percent $(\mathrm{n})$ \\
\hline Age (Years) & $51.6 \pm 15.6(22-87)$ \\
Gender (Male) & $65.6 . \%(40)$ \\
& \\
Race/Ethnicity & \\
White & $68.9 \%(42)$ \\
Black & $21.3 \%(13)$ \\
Latino & $3.3 \%(2)$ \\
Asian & $1.6 \%(1)$ \\
Native American & $1.6 \%(1)$ \\
Mixed Race/Ethnicity & $3.3 \%(2)$ \\
& \\
Education & \\
Years Completed & $13.6 \pm 3.1(4-19)$ \\
Less than 12 Years Completed & $18.0 \%(11)$ \\
\hline
\end{tabular}

Table 4.2 Cronbach's $\alpha$ for Global Function, Physical Function, and Psychosocial Function Variables

\begin{tabular}{lc}
\hline \multicolumn{1}{c}{ Variable } & Cronbach's $\alpha$ \\
\hline Global Function & 0.579 \\
SIP68 & \\
LLIP12 & \\
& \\
Physical Function & \\
SA & \\
MC & \\
MR & \\
& \\
Psychosocial Function & \\
PAC & \\
SB & \\
ES & \\
SIP68 = Sickness Impact Profile 68: Global Function \\
LLIP12 = Limb Loss Impact Profile 12: Global Function \\
SA = SIP68: Physical Function: Somatic Autonomy \\
MC = SIP68: Physical Function: Mobility Control \\
MR = SIP68: Physical Function: Mobility Range \\
PAC = SIP68: Psychosocial Function: Psychic Autonomy and Communication \\
SB = SIP68: Psychosocial Function: Social Behavior \\
ES = SIP68: Psychosocial Function: Emotional Stability
\end{tabular}


Table 4.3 Function Descriptive Data $(\mathrm{N}=61)$

\begin{tabular}{lc}
\hline \multicolumn{1}{c}{ Variable } & Mean \pm SD (Range) \\
\hline Global Function & \\
SIP68 & $19.7 \pm 14.5(1-54)$ \\
LLIP12 & $4.4 \pm 3.4(0-12)$ \\
Physical Function & \\
SA & \\
MC & $2.6 \pm 3.0(0-13)$ \\
MR & $6.0 \pm 3.5(0-12)$ \\
Psychosocial Function & $2.3 \pm 2.9(0-10)$ \\
PAC & \\
SB & \\
ES & $2.2 \pm 2.8(0-11)$ \\
\hline SIP68 Siln & $4.9 \pm 3.9(0-11)$ \\
\end{tabular}

SIP68 = Sickness Impact Profile 68: Global Function

LLIP12 = Limb Loss Impact Profile 12: Global Function

SA = SIP68: Physical Function: Somatic Autonomy

$\mathrm{MC}=$ SIP68: Physical Function: Mobility Control

MR = SIP68: Physical Function: Mobility Range

PAC = SIP68: Psychosocial Function: Psychic Autonomy and Communication

SB = SIP68: Psychosocial Function: Social Behavior

ES $=$ SIP68: Psychosocial Function: Emotional Stability 
Table 4.4 Pain Variable Descriptive Data by Location $(\mathrm{N}=61)^{*}$

\begin{tabular}{|c|c|c|c|}
\hline \multirow[b]{2}{*}{ Variable } & \multicolumn{3}{|c|}{ Mean \pm SD (Range) or Percent (n) } \\
\hline & PLP & RLP & OP \\
\hline Positive Pain Report & $83.6 \%(51)$ & $55.0 \%(33)$ & $62.7 \%(37)$ \\
\hline \multicolumn{4}{|l|}{ Intensity** } \\
\hline Current & $0.6 \pm 0.8(0-3)$ & $0.5 \pm 0.8(0-3)$ & $0.7 \pm 0.9(0-3)$ \\
\hline Usual & $1.1 \pm 0.9(0-3.5)$ & $0.8 \pm 1.1(0-3)$ & $.1 \pm 1.1(0-3.5)$ \\
\hline Worst & $2.5 \pm 1.4(0-4)$ & $1.7 \pm 1.7(0-4)$ & $2.0 \pm 1.7(0-4)$ \\
\hline Least & $0.2 \pm 0.5(0-2)$ & $0.2 \pm 0.5(0-2)$ & $0.4 \pm 0.7(0-3)$ \\
\hline \multicolumn{4}{|l|}{ Quality Descriptor } \\
\hline Burning & $40.7 \%(24)$ & $23.7 \%(14)$ & $25.4 \%(15)$ \\
\hline Cramping & $54.2 \%(32)$ & $25.4 \%(15)$ & $30.5 \%(18)$ \\
\hline Stabbing & $59.3 \%(35)$ & $32.2 \%(19)$ & $28.8 \%(17)$ \\
\hline Shocking/Shooting & $61.0 \%(36)$ & $27.1 \%(16)$ & $32.2 \%(19)$ \\
\hline Tiring & $33.9 \%(20)$ & $32.2 \%(19)$ & $33.9 \%(20)$ \\
\hline Twisting & $20.3 \%(12)$ & $13.6 \%(8)$ & $15.3 \%(9)$ \\
\hline Abnormal Position & $35.6 \%(21)$ & $16.9 \%(10)$ & $20.3 \%(12)$ \\
\hline Tight Band & $40.7 \%(24)$ & $22.0 \%(13)$ & $10.2 \%(6)$ \\
\hline \multicolumn{4}{|l|}{ Pattern } \\
\hline Intermittent & $83.1 \%(49)$ & $50.8 \%(30)$ & $50.8 \%(30)$ \\
\hline Continuous & $20.3 \%(12)$ & $13.6 \%(8)$ & $28.8 \%(17)$ \\
\hline \multicolumn{4}{|c|}{ PLP $=$ Phantom Limb Pain } \\
\hline \multicolumn{4}{|c|}{ RLP $=$ Residual Limb Pain } \\
\hline \multicolumn{4}{|l|}{$\mathrm{OP}=$ Other Pain } \\
\hline \multicolumn{4}{|c|}{$\begin{array}{l}* * \text { Pain intensity has been converted to the following numeric values: } 0=\text { No Pain, } 1= \\
\text { Small amount of pain, } 2=\text { Medium amount of pain, } 3=\text { Large amount of pain, } 4=\text { Pain } \\
\text { as bad as it can be. Pain intensity is reported as mean } \pm \text { standard deviation, with the } \\
\text { range in parenthesis. }\end{array}$} \\
\hline
\end{tabular}




\section{Table 4.5 Description of Pain Intensity Locations for Worst Other Pain} $(\mathrm{N}=59)$

\begin{tabular}{lll}
\hline \multicolumn{1}{c}{ Amputation Type } & Other Pain Areas & Percent (n) \\
\hline Upper Extremity & & \\
Unilateral & Sound Limb/Upper Extremity & $5.1 \%(3)$ \\
& Lower Back & $1.7 \%(1)$ \\
& No Other Pain & $1.7 \%(1)$ \\
& & \\
Bilateral & Neck/Upper Back & $1.7 \%(1)$ \\
& No Other Pain & $1.7 \%(1)$
\end{tabular}

Lower Extremity

Unilateral

Bilateral

$\begin{array}{ll}\text { Upper Extremity } & 20.3 \%(12) \\ \text { Neck/Upper Back/Shoulders } & 3.4 \%(2) \\ \text { Lower Back } & 6.8 \%(4) \\ \text { Ipsilateral Hip } & 1.7 \%(1) \\ \text { Sound Limb/Lower Extremity } & 8.5 \%(5) \\ \text { No Other Pain } & 27.1 \%(16) \\ & \\ \text { Neck/Upper back/ Shoulders } & 3.4 \%(2) \\ \text { Lower Back } & 3.4 \%(2) \\ \text { Buttocks } & 1.7 \%(1) \\ \text { No Other Pain } & 8.5 \%(5)\end{array}$

Upper Extremity

$20.3 \%(12)$

$3.4 \%(2)$

$6.8 \%(4)$

$1.7 \%(1)$

$8.5 \%(5)$

$27.1 \%(16)$

$3.4 \%(2)$

$3.4 \%(2)$

$8.5 \%(5)$

$\underline{\text { Both Upper and Lower }}$
Bilateral BEA/Bilateral AKA
Superficial Back of Head
$1.7 \%(1)$

Unilateral AEA/Hemi-

Upper Extremity

$1.7 \%(1)$

Pelvectomy

BEA $=$ Below the Elbow Amputation Level

$\mathrm{AEA}=$ Above the Elbow Amputation Level

$\mathrm{AKA}=$ Above the Knee Amputation Level 


\section{Health Status}

Health status factors and number of pain areas are reported in Table 4.6.

\section{Specific Aim 2}

Specific aim 2 estimated the relationship between function and pain, health status, and demographics in community-dwelling, adult Mid-Southerners with limb loss. The function variables are presented as global function (SIP68 total and LLIP12 total), physical function (SA, MC, and MR) and psychosocial function (PAC, SB, and ES). The results are described below.

\section{Correlations of Function with Pain}

The correlations of function and pain were presented by the pain locations of phantom limb, residual limb, and worst other pain. Pearson's $\mathrm{r}$ and $p$ values of function variables with pain variables are located in Tables 4.7 through 4.14.

\section{Correlation of Function with Phantom Limb Pain}

The correlations of the global function variables (SIP68 total and LLIP12 total) with PLP variables are described in this paragraph. The correlations of SIP68 total with phantom limb pain variables that were statistically significant were positive report of phantom limb pain, current PLP, usual PLP, worst PLP, stabbing PLP, tiring PLP, tight band PLP, and continuous PLP. These same variables were positively correlated with the LLIP12 total.

The correlations of the physical function variables (SA, MC, and MR) with PLP variables are described in this paragraph. The correlations of SA with phantom limb pain variables that were statistically significant include usual PLP, worst PLP, tiring PLP, tight band PLP, and continuous PLP. The correlations of MC with phantom limb pain variables that were statistically significant include positive report of phantom limb pain, usual PLP, worst PLP, stabbing PLP, tiring PLP, tight band PLP, and continuous PLP. The correlations of MR with phantom limb pain variables that were statistically significant include positive report of phantom limb pain, current PLP, usual PLP, worst PLP, least PLP, tiring PLP, tight band PLP, intermittent PLP, and continuous PLP.

The correlations of the psychosocial function variables (PAC, SB, and ES) with PLP variables are described in this paragraph. The correlations of PAC with phantom limb pain variables that were statistically significant were usual PLP, tiring PLP, and tight band PLP. The correlations of SB with phantom limb pain variables that were statistically significant were positive report of phantom limb pain, current PLP, usual PLP, worst PLP, stabbing PLP, tiring PLP, abnormal position PLP, tight band PLP, and 
Table 4.6 Health Status Descriptive Data $(\mathrm{N}=61)$ *

\begin{tabular}{|c|c|}
\hline Variable & Mean \pm SD (Range) or Percent (n) \\
\hline Diabetes & $31.1 \%(19)$ \\
\hline Length of Time with Diabetes (months) & $182.3 \pm 176.5(8-588)$ \\
\hline \multicolumn{2}{|l|}{ Smoking } \\
\hline Smoke Now & $16.4 \%(10)$ \\
\hline Ever Smoked & $54.1 \%(33)$ \\
\hline \multicolumn{2}{|l|}{ Obesity } \\
\hline BMI & $28.7 \pm 7.7(14.5-52.4)$ \\
\hline Obese & $37.7 \%(23)$ \\
\hline Overweight & $29.5 \%(18)$ \\
\hline Self Assessment of Health** & $2.8 \pm 0.6(1.5-4)$ \\
\hline \multicolumn{2}{|l|}{ Amputation History } \\
\hline \multicolumn{2}{|l|}{ Total Number of Limbs Lost } \\
\hline One Limb & $77.0 \%(47)$ \\
\hline Two Limbs & $21.3 \%(13)$ \\
\hline Three Limbs & $0 \%(0)$ \\
\hline Four Limbs & $1.6 \%(1)$ \\
\hline \multicolumn{2}{|l|}{ Amputation Type } \\
\hline Upper Limb(s) Only & $11.9 \%(7)$ \\
\hline \multicolumn{2}{|l|}{ Amputation Level } \\
\hline AEA/AKA & $48.3 \%(28)$ \\
\hline \multicolumn{2}{|l|}{ Amputation Duration } \\
\hline Last Reported Amputation Surgery (months) & $104.7 \pm 128.4(6-467)$ \\
\hline \multicolumn{2}{|l|}{ Amputation Cause } \\
\hline Traumatic & $45.9 \%(28)$ \\
\hline Number of Pain Areas ${ }^{* * *}$ & $5.4 \pm 4.2(0-21)$ \\
\hline
\end{tabular}

BMI = Body Mass Index

AEA/AKA = Above the Elbow Amputation Level /Above the Knee Amputation Level ${ }^{*} \mathrm{n}=60$ for number of pain areas due to missing data

${ }^{* *}$ Self assessment of health has been converted to the following numeric values: $0=$ Bad, $1=$ Poor, $2=$ Fair, $3=$ Good, $4=$ Excellent. $* * *$ The sum of pain areas out of a possible 36 using the method described by Escalante et al (Escalante A, Lichtenstein MJ, White K, Rios N, Hazuda HP. A method for scoring the pain map of the McGill Pain Questionnaire for use in epidemiologic studies. Aging-Clinical \& Experimental Research 7(5):358-66, 1995 Oct.). 
Table 4.7 Correlations of Global Function (SIP68 Total) with Pain Variables by Pain Location

\begin{tabular}{|c|c|c|c|c|c|c|}
\hline \multirow{3}{*}{$\begin{array}{c}\text { Pain } \\
\text { Variable }\end{array}$} & \multicolumn{6}{|c|}{ Pain Location } \\
\hline & \multicolumn{2}{|c|}{$\underline{\text { PLP }}$} & \multicolumn{2}{|c|}{$\underline{\text { RLP }}$} & \multicolumn{2}{|c|}{$\underline{\mathrm{OP}}$} \\
\hline & $\mathrm{r}$ & $p$ & $\mathrm{r}$ & $p$ & $\mathrm{r}$ & $p$ \\
\hline Positive Pain Report & $0.36^{*}$ & .005 & 0.15 & .239 & 0.25 & .053 \\
\hline \multicolumn{7}{|l|}{ Intensity } \\
\hline Current & $0.35^{*}$ & .006 & 0.19 & .157 & $0.30^{*}$ & .022 \\
\hline Usual & $0.62 *$ & $<.001$ & 0.23 & .078 & $0.39 *$ & .002 \\
\hline Worst & $0.43 *$ & .001 & 0.10 & .473 & 0.24 & .068 \\
\hline Least & 0.25 & .053 & 0.11 & .423 & 0.21 & .114 \\
\hline \multicolumn{7}{|l|}{ Quality Descriptor } \\
\hline Burning & 0.10 & .453 & 0.23 & .082 & 0.21 & .106 \\
\hline Cramping & 0.15 & .268 & $0.26 *$ & .049 & $0.29 *$ & .026 \\
\hline Stabbing & $0.33 *$ & .012 & 0.26 & .051 & $0.26^{*}$ & .046 \\
\hline Shocking/Shooting & 0.21 & .120 & 0.15 & .251 & 0.08 & .537 \\
\hline Tiring & $0.43 *$ & .001 & 0.09 & .484 & $0.36^{*}$ & .005 \\
\hline Twisting & 0.13 & .340 & 0.09 & .484 & 0.23 & .085 \\
\hline Abnormal Position & 0.25 & .060 & $0.26^{*}$ & .044 & $0.30^{*}$ & .023 \\
\hline Tight Band & $0.45^{*}$ & $<.001$ & $0.39 *$ & .003 & 0.13 & .338 \\
\hline \multicolumn{7}{|l|}{ Pattern } \\
\hline Intermittent & 0.23 & .077 & 0.16 & .228 & 0.23 & .081 \\
\hline Continuous & $0.38 *$ & .003 & 0.15 & .271 & $0.26^{*}$ & .046 \\
\hline $\begin{array}{l}\text { PLP }=\text { Phantom Limb } \\
\text { RLP = Residual Limb } \\
\text { OP = Other Pain } \\
* \text { Significant at the } 0.0\end{array}$ & $\begin{array}{l}\text { ain } \\
\text { ain }\end{array}$ & & & & & \\
\hline
\end{tabular}


Table 4.8 Correlations of Global Function Attributed Specifically to Limb Loss (LLIP12 Total) with Pain Variables by Pain Location

\begin{tabular}{|c|c|c|c|c|c|c|}
\hline \multirow{3}{*}{$\begin{array}{c}\text { Pain } \\
\text { Variable }\end{array}$} & \multicolumn{6}{|c|}{ Pain Location } \\
\hline & \multicolumn{2}{|c|}{$\underline{\text { PLP }}$} & \multicolumn{2}{|c|}{$\underline{\text { RLP }}$} & \multicolumn{2}{|c|}{$\underline{\mathrm{OP}}$} \\
\hline & $\mathrm{r}$ & $p$ & $\mathrm{r}$ & $p$ & $\mathrm{r}$ & $p$ \\
\hline Positive Pain Report & $0.39 *$ & .002 & 0.25 & .057 & $0.26^{*}$ & .050 \\
\hline \multicolumn{7}{|l|}{ Intensity } \\
\hline Current & $0.39 *$ & .002 & 0.25 & .053 & $0.31 *$ & .018 \\
\hline Usual & $0.59 *$ & $<.001$ & $0.30 *$ & .023 & $0.44 *$ & .001 \\
\hline Worst & $0.46^{*}$ & $<.001$ & 0.22 & .101 & $0.29 *$ & .026 \\
\hline Least & 0.19 & 0.161 & 0.05 & .717 & 0.21 & .104 \\
\hline \multicolumn{7}{|l|}{ Quality Descriptor } \\
\hline Burning & 0.12 & .355 & 0.22 & .090 & 0.22 & .101 \\
\hline Cramping & 0.19 & .143 & $0.30 *$ & .020 & $0.42 *$ & .001 \\
\hline Stabbing & $0.34 *$ & .008 & $0.35 *$ & .006 & $0.27 *$ & .036 \\
\hline Shocking/Shooting & 0.23 & .074 & 0.17 & .188 & 0.13 & .341 \\
\hline Tiring & $0.34^{*}$ & .009 & 0.14 & .299 & $0.30 *$ & .020 \\
\hline Twisting & 0.24 & .068 & 0.18 & .166 & $0.32 *$ & .014 \\
\hline Abnormal Position & 0.24 & .065 & $0.30 *$ & .019 & $0.32 *$ & .014 \\
\hline Tight Band & $0.51 *$ & .001 & $0.44^{*}$ & .001 & $0.27^{*}$ & .043 \\
\hline \multicolumn{7}{|l|}{ Pattern } \\
\hline Intermittent & 0.23 & .075 & 0.24 & .069 & 0.23 & .083 \\
\hline Continuous & $0.39 *$ & .003 & 0.23 & .081 & $0.26^{*}$ & .045 \\
\hline
\end{tabular}

PLP $=$ Phantom Limb Pain

RLP $=$ Residual Limb Pain

$\mathrm{OP}=$ Other Pain

*Significant at the 0.05 level 
Table 4.9 Correlations of Physical Function (Somatic Autonomy) with Pain Variables by Pain Location

\begin{tabular}{|c|c|c|c|c|c|c|}
\hline \multirow{3}{*}{$\begin{array}{c}\text { Pain } \\
\text { Variable }\end{array}$} & \multicolumn{6}{|c|}{ Pain Location } \\
\hline & \multicolumn{2}{|c|}{$\underline{\text { PLP }}$} & \multicolumn{2}{|c|}{$\underline{\mathrm{RLP}}$} & \multicolumn{2}{|c|}{$\underline{\mathrm{OP}}$} \\
\hline & $\mathrm{r}$ & $p$ & r & $p$ & $\mathrm{r}$ & $p$ \\
\hline Positive Pain Report & 0.24 & .059 & .097 & .460 & 0.23 & .081 \\
\hline \multicolumn{7}{|l|}{ Intensity } \\
\hline Current & 0.13 & .320 & 0.15 & .266 & $0.33^{*}$ & .012 \\
\hline Usual & $0.40 *$ & .002 & 0.10 & .473 & $0.32 *$ & .013 \\
\hline Worst & $0.33 *$ & .010 & 0.03 & .851 & 0.15 & .265 \\
\hline Least & 0.11 & .392 & 0.07 & .584 & 0.14 & .282 \\
\hline \multicolumn{7}{|l|}{ Quality Descriptor } \\
\hline Burning & -0.05 & .721 & 0.14 & .292 & $0.34 *$ & .008 \\
\hline Cramping & 0.09 & .511 & 0.18 & .182 & 0.09 & .478 \\
\hline Stabbing & 0.22 & .092 & 0.12 & .382 & 0.22 & .098 \\
\hline Shocking/Shooting & 0.11 & .405 & 0.02 & .885 & -0.08 & .541 \\
\hline Tiring & $0.35^{*}$ & .007 & 0.10 & .442 & 0.25 & .058 \\
\hline Twisting & 0.01 & .929 & 0.12 & .372 & 0.07 & .601 \\
\hline Abnormal Position & -0.03 & .798 & 0.24 & .072 & 0.06 & .647 \\
\hline Tight Band & $0.29 *$ & .027 & $0.26^{*}$ & .047 & -0.03 & .853 \\
\hline \multicolumn{7}{|l|}{ Pattern } \\
\hline Intermittent & 0.15 & .256 & 0.03 & .818 & 0.25 & .052 \\
\hline Continuous & $0.26^{*}$ & .049 & 0.21 & .103 & $0.26 *$ & .046 \\
\hline
\end{tabular}

PLP $=$ Phantom Limb Pain

RLP $=$ Residual Limb Pain

$\mathrm{OP}=$ Other Pain

*Significant at the 0.05 level 
Table 4.10 Correlations of Physical Function (Mobility Control) with Pain Variables by Pain Location

\begin{tabular}{|c|c|c|c|c|c|c|}
\hline \multirow{3}{*}{$\begin{array}{c}\text { Pain } \\
\text { Variable }\end{array}$} & \multicolumn{6}{|c|}{ Pain Location } \\
\hline & \multicolumn{2}{|c|}{$\underline{\text { PLP }}$} & \multicolumn{2}{|c|}{$\underline{\mathrm{RLP}}$} & \multicolumn{2}{|c|}{$\underline{\mathrm{OP}}$} \\
\hline & $\mathrm{r}$ & $p$ & $\mathrm{r}$ & $p$ & $\mathrm{r}$ & $p$ \\
\hline Positive Pain Report & $0.36 *$ & .004 & 0.12 & .366 & 0.25 & .061 \\
\hline \multicolumn{7}{|l|}{ Intensity } \\
\hline Current & 0.17 & .202 & 0.09 & .489 & 0.22 & .101 \\
\hline Usual & $0.49 *$ & $<.001$ & 0.15 & .251 & $0.33^{*}$ & .012 \\
\hline Worst & $0.36^{*}$ & .005 & 0.04 & .749 & 0.18 & .170 \\
\hline Least & 0.15 & .247 & 0.04 & .742 & 0.10 & .441 \\
\hline \multicolumn{7}{|l|}{ Quality Descriptor } \\
\hline Burning & 0.10 & .436 & $0.31 *$ & .016 & 0.22 & .100 \\
\hline Cramping & 0.16 & .220 & 0.22 & .100 & 0.20 & .133 \\
\hline Stabbing & $0.32 *$ & .014 & $0.26 *$ & .047 & 0.20 & .129 \\
\hline Shocking/Shooting & 0.16 & .223 & 0.11 & .394 & -0.02 & .910 \\
\hline Tiring & $0.27 *$ & .040 & 0.09 & .494 & $0.33 *$ & .010 \\
\hline Twisting & -0.02 & .899 & 0.13 & .322 & $0.31 *$ & .019 \\
\hline Abnormal Position & 0.16 & .215 & $0.27 *$ & .040 & 0.23 & .081 \\
\hline Tight Band & $0.42 *$ & .001 & $0.28 *$ & .030 & 0.13 & .335 \\
\hline \multicolumn{7}{|l|}{ Pattern } \\
\hline Intermittent & $0.29 *$ & .028 & 0.14 & .293 & 0.24 & .069 \\
\hline Continuous & $0.27 *$ & .042 & 0.12 & .378 & 0.22 & .091 \\
\hline $\begin{array}{l}\text { PLP }=\text { Phantom Limb } \\
\text { RLP }=\text { Residual Limb } \\
\text { OP }=\text { Other Pain } \\
* \text { Significant at the } 0.0\end{array}$ & $\begin{array}{l}\text { ain } \\
\text { ain }\end{array}$ & & & & & \\
\hline
\end{tabular}


Table 4.11 Correlations of Physical Function (Mobility Range) with Pain Variables by Pain Location

\begin{tabular}{|c|c|c|c|c|c|c|}
\hline \multirow{3}{*}{$\begin{array}{c}\text { Pain } \\
\text { Variable }\end{array}$} & \multicolumn{6}{|c|}{ Pain Location } \\
\hline & \multicolumn{2}{|c|}{$\underline{\text { PLP }}$} & \multicolumn{2}{|c|}{$\underline{\text { RLP }}$} & \multicolumn{2}{|c|}{$\underline{\mathrm{OP}}$} \\
\hline & $\mathrm{r}$ & $p$ & $\mathrm{r}$ & $p$ & $\mathrm{r}$ & $p$ \\
\hline Positive Pain Report & $0.34^{*}$ & .007 & 0.01 & .466 & 0.12 & .361 \\
\hline \multicolumn{7}{|l|}{ Intensity } \\
\hline Current & $0.37 *$ & .004 & 0.07 & .601 & $0.26^{*}$ & .050 \\
\hline Usual & $0.52 *$ & $<.001$ & 0.10 & .435 & $0.28 *$ & .031 \\
\hline Worst & $0.30 *$ & .022 & 0.02 & .894 & 0.13 & .315 \\
\hline Least & $0.31 *$ & .016 & 0.15 & .258 & 0.22 & .094 \\
\hline \multicolumn{7}{|l|}{ Quality Descriptor } \\
\hline Burning & -0.05 & .683 & 0.03 & .832 & 0.10 & .442 \\
\hline Cramping & 0.097 & .467 & 0.18 & .178 & 0.18 & .175 \\
\hline Stabbing & 0.22 & .101 & 0.19 & .151 & 0.18 & .165 \\
\hline Shocking/Shooting & 0.15 & .262 & 0.16 & .232 & 0.15 & .267 \\
\hline Tiring & $0.42 *$ & .001 & 0.03 & .800 & $0.27 *$ & .039 \\
\hline Twisting & 0.23 & .080 & 0.08 & .530 & $0.30 *$ & .022 \\
\hline Abnormal Position & 0.16 & .242 & 0.25 & .057 & 0.21 & .105 \\
\hline Tight Band & $0.39 *$ & .002 & $0.33 *$ & .010 & 0.08 & .566 \\
\hline \multicolumn{7}{|l|}{ Pattern } \\
\hline Intermittent & $0.26^{*}$ & .050 & 0.11 & .400 & -0.02 & .878 \\
\hline Continuous & $0.46^{*}$ & $<.001$ & 0.01 & .963 & $0.30 *$ & .021 \\
\hline $\begin{array}{l}\text { PLP }=\text { Phantom Limb } \\
\text { RLP = Residual Limb } \\
\text { OP = Other Pain } \\
* \text { Significant at the } 0.0\end{array}$ & $\begin{array}{l}\text { in } \\
\text { iin }\end{array}$ & & & & & \\
\hline
\end{tabular}


Table 4.12 Correlations of Psychosocial Function (Psychic Autonomy and Communication) with Pain Variables by Pain Location

\begin{tabular}{|c|c|c|c|c|c|c|}
\hline \multirow{3}{*}{$\begin{array}{c}\text { Pain } \\
\text { Variable }\end{array}$} & \multicolumn{6}{|c|}{ Pain Location } \\
\hline & \multicolumn{2}{|c|}{$\underline{\text { PLP }}$} & \multicolumn{2}{|c|}{$\underline{\text { RLP }}$} & \multicolumn{2}{|c|}{$\underline{\mathrm{OP}}$} \\
\hline & $\mathrm{r}$ & $p$ & $\mathrm{r}$ & $p$ & $\mathrm{r}$ & $p$ \\
\hline Positive Pain Report & 0.21 & .097 & 0.21 & .106 & 0.12 & .379 \\
\hline \multicolumn{7}{|l|}{ Intensity } \\
\hline Current & 0.19 & .147 & 0.19 & .153 & 0.16 & .230 \\
\hline Usual & $0.45^{*}$ & $<.001$ & 0.24 & .063 & 0.24 & .062 \\
\hline Worst & 0.23 & .078 & 0.14 & .303 & 0.16 & .222 \\
\hline Least & 0.21 & .113 & 0.09 & .498 & 0.17 & .213 \\
\hline \multicolumn{7}{|l|}{ Quality Descriptor } \\
\hline Burning & 0.05 & .713 & 0.17 & .196 & 0.14 & .300 \\
\hline Cramping & 0.06 & .676 & 0.24 & .063 & $0.29 *$ & .025 \\
\hline Stabbing & 0.15 & .246 & 0.16 & .226 & 0.13 & .312 \\
\hline Shocking/Shooting & 0.12 & .387 & 0.15 & .256 & 0.05 & .726 \\
\hline Tiring & $0.29 *$ & .029 & 0.16 & .226 & 0.20 & .127 \\
\hline Twisting & 0.11 & .397 & 0.03 & .822 & 0.14 & .289 \\
\hline Abnormal Position & 0.24 & .066 & 0.22 & .088 & 0.23 & .083 \\
\hline Tight Band & $0.37 *$ & .004 & $0.43 *$ & .001 & 0.05 & .691 \\
\hline \multicolumn{7}{|l|}{ Pattern } \\
\hline Intermittent & 0.06 & .659 & 0.21 & .106 & 0.17 & .191 \\
\hline Continuous & 0.20 & .140 & 0.13 & .339 & 0.08 & .570 \\
\hline $\begin{array}{l}\text { PLP }=\text { Phantom Limb } \\
\text { RLP }=\text { Residual Limb } \\
\text { OP = Other Pain } \\
* \text { Significant at the } 0.0\end{array}$ & $\begin{array}{l}n \\
n\end{array}$ & & & & & \\
\hline
\end{tabular}


Table 4.13 Correlations of Psychosocial Function (Social Behavior) with Pain Variables by Pain Location

\begin{tabular}{|c|c|c|c|c|c|c|}
\hline \multirow{3}{*}{$\begin{array}{c}\text { Pain } \\
\text { Variable }\end{array}$} & \multicolumn{6}{|c|}{ Pain Location } \\
\hline & \multicolumn{2}{|c|}{$\underline{\text { PLP }}$} & \multicolumn{2}{|c|}{$\underline{\text { RLP }}$} & \multicolumn{2}{|c|}{$\underline{\mathrm{OP}}$} \\
\hline & $\mathrm{r}$ & $p$ & r & $p$ & $r$ & $p$ \\
\hline Positive Pain Report & $0.35 *$ & .006 & 0.1 & .446 & 0.25 & .056 \\
\hline \multicolumn{7}{|l|}{ Intensity } \\
\hline Current & $0.41^{*}$ & .001 & 0.18 & .178 & $0.26^{*}$ & .047 \\
\hline Usual & $0.60 *$ & $<.001$ & 0.24 & .066 & $0.37 *$ & .004 \\
\hline Worst & $0.46^{*}$ & $<.001$ & 0.09 & .497 & $0.27 *$ & .042 \\
\hline Least & 0.25 & .058 & 0.08 & .551 & 0.18 & .169 \\
\hline \multicolumn{7}{|l|}{ Quality Descriptor } \\
\hline Burning & 0.17 & .202 & 0.18 & .181 & 0.07 & .585 \\
\hline Cramping & 0.14 & .288 & 0.19 & .159 & $0.29 *$ & .026 \\
\hline Stabbing & $0.33 *$ & .010 & 0.24 & .066 & $0.29 *$ & .025 \\
\hline Shocking/Shooting & 0.20 & .130 & 0.16 & .214 & 0.15 & .273 \\
\hline Tiring & $0.44 *$ & .001 & 0.02 & .877 & $0.35 *$ & .006 \\
\hline Twisting & 0.18 & .171 & 0.02 & .909 & 0.18 & .173 \\
\hline Abnormal Position & $0.32 *$ & .015 & 0.14 & .292 & $0.33^{*}$ & .012 \\
\hline Tight Band & $0.34 *$ & .008 & $0.28^{*}$ & .035 & 0.22 & .100 \\
\hline \multicolumn{7}{|l|}{ Pattern } \\
\hline Intermittent & 0.24 & .065 & 0.11 & .413 & 0.20 & .133 \\
\hline Continuous & $0.33 *$ & .012 & 0.08 & .544 & 0.17 & .188 \\
\hline $\begin{array}{l}\text { PLP }=\text { Phantom Limb } \\
\text { RLP = Residual Limb } \\
\text { OP }=\text { Other Pain } \\
* \text { Significant at the } 0.0\end{array}$ & ain & & & & & \\
\hline
\end{tabular}


Table 4.14 Correlations of SIP68 Psychosocial Function (Emotional Stability) with Pain Variables by Pain Location

\begin{tabular}{|c|c|c|c|c|c|c|}
\hline \multirow{3}{*}{$\begin{array}{c}\text { Pain } \\
\text { Variable }\end{array}$} & \multicolumn{6}{|c|}{ Pain Location } \\
\hline & \multicolumn{2}{|c|}{$\underline{\text { PLP }}$} & \multicolumn{2}{|c|}{$\underline{\mathrm{RLP}}$} & \multicolumn{2}{|c|}{$\underline{\mathrm{OP}}$} \\
\hline & $\mathrm{r}$ & - $p$ & $\mathrm{r}$ & $p$ & $\mathrm{r}$ & $p$ \\
\hline Positive Pain Report & 0.15 & 0.25 & 0.15 & .248 & 0.21 & .106 \\
\hline \multicolumn{7}{|l|}{ Intensity } \\
\hline Current & $0.48^{*}$ & $<.001$ & $0.27 *$ & .041 & 0.18 & .184 \\
\hline Usual & $0.49 *$ & $<.001$ & $0.30 *$ & .023 & 0.25 & .060 \\
\hline Worst & $0.36^{*}$ & .004 & 0.19 & .148 & 0.25 & .062 \\
\hline Least & 0.15 & .254 & 0.09 & .491 & 0.21 & .104 \\
\hline \multicolumn{7}{|l|}{ Quality Descriptor } \\
\hline Burning & $0.29 *$ & .029 & 0.25 & .058 & 0.18 & .174 \\
\hline Cramping & 0.14 & .291 & 0.25 & .054 & $0.36^{*}$ & .005 \\
\hline Stabbing & 0.26 & .051 & 0.23 & .082 & 0.17 & .201 \\
\hline Shocking/Shooting & $0.27 *$ & .041 & 0.09 & .497 & 0.18 & .166 \\
\hline Tiring & 0.21 & .106 & 0.05 & .726 & $0.26^{*}$ & .049 \\
\hline Twisting & 0.08 & .540 & 0.07 & .577 & -0.04 & .792 \\
\hline Abnormal Position & $0.35^{*}$ & .006 & 0.12 & .366 & $0.37 *$ & .004 \\
\hline Tight Band & $0.33^{*}$ & .011 & $0.27 *$ & .039 & 0.09 & .501 \\
\hline \multicolumn{7}{|l|}{ Pattern } \\
\hline Intermittent & 0.05 & .708 & 0.19 & .161 & 0.25 & .058 \\
\hline Continuous & $0.32 *$ & .014 & 0.20 & .133 & 0.24 & .068 \\
\hline $\begin{array}{l}\text { PLP }=\text { Phantom Limb } \\
\text { RLP }=\text { Residual Limb } \\
\text { OP }=\text { Other Pain } \\
* \text { Significant at the } 0.0\end{array}$ & $\begin{array}{l}\text { Pain } \\
\text { Pain }\end{array}$ & & & & & \\
\hline
\end{tabular}


continuous PLP. The correlations of ES with phantom limb pain variables that were statistically significant were current PLP, usual PLP, worst PLP, burning PLP, shocking/shooting PLP, abnormal position PLP, tight band PLP, and continuous PLP.

\section{Correlation of Function with Residual Limb Pain}

The correlations of the global function variables (SIP68 total and LLIP12 total) with RLP variables are described in this section. The correlations of the SIP68 total with residual limb pain variables that were statistically significant include cramping RLP, abnormal position RLP, and tight band RLP. The correlations of the LLIP12 with residual limb pain variables that were statistically significant include usual RLP, cramping RLP, stabbing RLP, abnormal position RLP, and tight band RLP.

The correlations of the physical function variables (SA, MC, and MR) with RLP variables are described in this paragraph. The only significant correlation with SA was tight band. The correlations of MC with residual limb pain that were statistically significant include burning RLP, stabbing RLP, abnormal position RLP, and tight band RLP. The only significant correlation with MR was residual limb pain variables was tight band RLP.

The correlations of the psychosocial function variables (PAC, SB, and ES) with RLP variables are described in this paragraph. The only significant correlation with PAC and SB was tight band RLP. The significant correlations with ES were current RLP, usual RLP, and tight band pain RLP.

\section{Correlation of Function with Other Pain}

The correlations of the global function variables (SIP68 total and LLIP12 total) with OP variables are described in this paragraph. The correlations of the SIP68 total with other pain variables that were statistically significant include current OP, usual OP, cramping OP, stabbing OP, tiring OP, abnormal position OP, and continuous OP. The correlations of the LLIP12 with other pain variables that were statistically significant include positive report of OP, current OP, usual OP, worst OP, cramping OP, stabbing $\mathrm{OP}$, tiring OP, twisting OP, abnormal position OP, tight band OP, and continuous.

The correlations of the physical function variables (SA, MC, and MR) with OP variables are described in this paragraph. The correlations of SA with other pain variables that were statistically significant include current OP, usual OP, burning OP, and continuous $\mathrm{OP}$. The correlations of $\mathrm{MC}$ with other pain variables that were statistically significant include usual OP, and tiring OP. The correlations of MR with other pain variables that were statistically significant include current OP usual OP, tiring $\mathrm{OP}$, twisting OP, and continuous OP.

The correlations of the psychosocial function variables (PAC, SB, and ES) with $\mathrm{OP}$ variables are described in this paragraph. The only significant correlation of PAC with other pain variables was cramping OP. The correlations of SB with other pain 
variables that were significant include current OP, usual OP, worst OP, cramping OP, stabbing OP, tiring OP, and abnormal position OP. The correlations of ES with other pain variables that were statistically significant were cramping $O P$, tiring $O P$, and abnormal position OP.

\section{Correlations of Function with Health Status}

Correlations of function and health status are presented by the health status variable of diabetes, smoking, self-assessment of health, and amputation history. Pearson's $r$ and $p$ values of function variables with pain variables are located in Tables 4.15 through 4.17.

\section{Correlation of Function with Diabetes, Smoking, Obesity, and Self Assessment of Health}

Diabetes was not correlated with any of the function variables. Smoking had a positive significant correlation with PAC. There were no other function variables correlated with smoking. BMI was not correlated with any of the function variables. Self-assessment of health (SAH) had a significant negative correlation with MC. There were no other function variables correlated with SAH.

\section{Correlation of Function with Amputation History}

The correlations of the global function variables (SIP68 total and LLIP12 total) with amputation history variables are described in this paragraph. Amputation duration had a significant correlation with both the global function variables SIP68 and LLIP12. The correlations of the physical function variables (SA, MC, and MR) amputation history variables are described in this paragraph. The correlations of SA with health status variables that were statistically significant include number of limbs lost and amputation cause: traumatic. The correlations of $\mathrm{MC}$ with health status variables that were statistically significant include amputation type: lower extremity, amputation duration, and amputation cause: traumatic. The correlations of MR with health status variables that were statistically significant include amputation type: lower extremity and amputation duration.

The correlations of the psychosocial function variables (PAC, SB, and ES) with amputation history variables are described in this paragraph. The only significant correlation amputation history and psychosocial function variables was SB with amputation duration. 
Table 4.15 Correlations of Global Function (SIP68 Total and LLIP12 Total) with Health Status Variables

\begin{tabular}{lcccc}
\hline \multicolumn{1}{c}{ Variable } & \multicolumn{2}{c}{ SIP68 Total } & \multicolumn{2}{c}{ LLIP12 Total } \\
\hline Diabetes & $\mathrm{r}$ & $p$ & 0.13 & .317 \\
Smoking & 0.13 & .335 & & \\
$\quad$ Smoke Now & & & & \\
Ever Smoked & 0.10 & .462 & 0.14 & .268 \\
& 0.04 & .762 & 0.03 & .823 \\
Obesity: BMI & & & & \\
& 0.10 & .451 & 0.21 & .097 \\
Self Assessment of Health & -0.21 & .113 & -0.22 & .088 \\
& & & & \\
Amputation History & & & & \\
Number of Limbs Lost & 0.13 & .322 & -0.02 & .876 \\
Type: Lower Extremity & 0.25 & .055 & 0.25 & .061 \\
Level: AEA/AKA & -0.15 & .256 & -0.06 & .677 \\
Amputation Duration & $-0.39 *$ & .003 & $-0.40 *$ & .001 \\
Cause: Traumatic & -0.20 & .131 & -0.14 & .298 \\
Number of Pain Areas & $0.34^{*}$ & .007 & $0.39 *$ & .002 \\
\hline
\end{tabular}

SIP68 = Sickness Impact Profile 68: Global Function

LLIP12 = Limb Loss Impact Profile 12: Global Function

$\mathrm{BMI}=$ Body Mass Index

AEA/AKA $=$ Above the Elbow Amputation Level /Above the Knee Amputation Level

*Significant at the 0.05 level 
Table 4.16 Correlations of SIP68 Physical Function with Health Status Variables and Number of Pain Areas

\begin{tabular}{|c|c|c|c|c|c|c|}
\hline \multirow[b]{2}{*}{ Variable } & \multicolumn{2}{|c|}{$\underline{\mathrm{SA}}$} & \multicolumn{2}{|c|}{$\underline{\mathrm{MC}}$} & \multicolumn{2}{|c|}{$\underline{\mathrm{MR}}$} \\
\hline & $\mathrm{r}$ & $p$ & $\mathrm{r}$ & $p$ & $\mathrm{r}$ & $p$ \\
\hline Diabetes & 0.07 & .572 & 0.17 & .195 & 0.09 & .484 \\
\hline \multicolumn{7}{|l|}{ Smoking } \\
\hline Smoke Now & -0.06 & .627 & -0.06 & .633 & 0.05 & .677 \\
\hline Ever Smoked & -0.02 & .902 & -0.03 & .800 & -0.06 & .640 \\
\hline Obesity: BMI & -0.02 & .872 & 0.18 & .176 & 0.06 & .652 \\
\hline $\begin{array}{l}\text { Self Assessment of } \\
\text { Health }\end{array}$ & -0.03 & .794 & $-0.31 *$ & .015 & -0.13 & .337 \\
\hline \multicolumn{7}{|l|}{ Amputation History } \\
\hline Number of Limbs Lost & $0.45 *$ & $<.001$ & 0.08 & .531 & 0.07 & .602 \\
\hline Type: Lower Extremity & 0.22 & .099 & $0.34^{*}$ & .009 & $0.26^{*}$ & .047 \\
\hline Level: AEA/AKA & -0.23 & .089 & -0.16 & .240 & -0.09 & 0.499 \\
\hline Amputation Duration & -0.24 & .066 & $-0.49 *$ & $<.001$ & $-0.35^{*}$ & .006 \\
\hline Cause: Traumatic & $-0.32 *$ & .013 & $-0.27 *$ & .033 & -0.22 & .083 \\
\hline Number of Pain Areas & $0.28 *$ & .031 & $0.30 *$ & .018 & 0.23 & .074 \\
\hline
\end{tabular}

SA = SIP68: Physical Function: Somatic Autonomy

$\mathrm{MC}=$ SIP68: Physical Function: Mobility Control

MR = SIP68: Physical Function: Mobility Range

$\mathrm{BMI}=$ Body Mass Index

AEK/AKA = Above the Elbow Amputation Level /Above the Knee Amputation Level *Significant at the 0.05 level 
Table 4.17 Correlations of SIP68 Psychosocial Function with Health Status Variables

\begin{tabular}{|c|c|c|c|c|c|c|}
\hline \multirow[b]{2}{*}{ Variable } & \multicolumn{2}{|c|}{$\underline{\mathrm{PAC}}$} & \multicolumn{2}{|c|}{$\underline{\text { SB }}$} & \multicolumn{2}{|c|}{$\underline{E S}$} \\
\hline & $r$ & $p$ & $r$ & $p$ & $r$ & $p$ \\
\hline Diabetes & 0.16 & .233 & 0.04 & .768 & 0.10 & .452 \\
\hline Smoking & & & & & & \\
\hline Smoke Now & $0.29 *$ & .021 & 0.11 & .399 & 0.23 & .077 \\
\hline Ever Smoked & 0.17 & .199 & 0.06 & .643 & 0.07 & .605 \\
\hline Obesity: BMI & 0.03 & .836 & 0.12 & .359 & 0.10 & .455 \\
\hline $\begin{array}{l}\text { Self Assessment of } \\
\text { Health }\end{array}$ & -0.09 & .511 & -0.23 & .070 & -0.17 & .203 \\
\hline Amputation History & & & & & & \\
\hline Number of Limbs Lost & -0.06 & .655 & 0.05 & .718 & 0.01 & .943 \\
\hline Type: Lower Extremity & 0.08 & .558 & 0.20 & .137 & 0.05 & .714 \\
\hline Level: AEA/AKA & -0.18 & .169 & -0.05 & .696 & 0.01 & .946 \\
\hline Amputation Duration & -0.12 & .367 & $-0.36^{*}$ & .005 & -0.11 & .387 \\
\hline Cause: Traumatic & -0.08 & .519 & -0.001 & .992 & -0.03 & .834 \\
\hline Number of Pain Areas & 0.18 & .176 & $0.33 *$ & .011 & $0.33 *$ & .011 \\
\hline
\end{tabular}

PAC = SIP68: Psychosocial Function: Psychic Autonomy and Communication $\mathrm{SB}=$ SIP68: Psychosocial Function: Social Behavior ES = SIP68: Psychosocial Function: Social Behavior AEK/AKA = Above the Elbow Amputation Level /Above the Knee Amputation Level *Significant at the 0.05 level 


\section{Correlations of Function with Demographics}

The correlations of function and demographics are presented by age, male gender, Non-White race/ethnicity, and education. Pearson's $\mathrm{r}$ and $p$ values of function variables with pain variables are located in Tables 4.18 through 4.21.

\section{Correlation of Function with Age, Gender, Race/Ethnicity}

Age had a significant positive correlation with SA and MC. Male gender was not correlated with any function variables in this study. Non-White race/ethnicity had a significant positive correlation with SIP68, SA, MR, PAC, and SB.

\section{Correlation of Function with Education}

Education had a negative significant correlation with SA and showed a trend toward a negative relationship with MR.

\section{Specific Aim 3}

Specific aim 3 was to estimate the joint influences on function by pain, health status, and demographics of community-dwelling, adult Mid-Southerners with limb loss. The outcome variables chosen for the model analyses with multiple regression included SIP68, MR, and SB. The predictor candidates were usual PLP, tiring OP, tight band OP, intermittent PLP, continuous PLP, usual OP, abnormal position OP, amputation type: lower extremity, and Non-White race/ethnicity.

\section{Model to Predict Global Function (SIP68 Total)}

In multiple linear regressions, the candidate variables explained $52 \%$ of the variability of SIP68: global function. Variables with the greatest magnitude in the model include usual PLP $(\beta=0.27, p=.096)$, tight band PLP $(\beta=0.26, p=.028)$, Non-White race/ethnicity $(\beta=0.20, p=.076)$ and continuous $\operatorname{PLP}(\beta=0.16, p=.216)$. Table 4.21 summarizes the results of the multiple regression.

\section{Model to Predict Physical Function (Mobility Range)}

In multiple linear regressions, the candidate variables explained $51 \%$ of the variability of the SIP68 physical function: mobility range. Variables with the greatest magnitude in the model include continuous PLP $(\beta=0.29, p=.028)$, Non-White race/ethnicity $(\beta=0.24, p=.039)$, tight band PLP $(\beta=0.22, p=.062)$, and intermittent OP $(\beta=-0.22, p=.093)$. Table 4.22 summarizes the results of the multiple regression. 
Table 4.18 Correlations of SIP68 Global Function Totals with Demographic Variables

\begin{tabular}{|c|c|c|c|c|}
\hline \multirow[b]{2}{*}{ Variable } & \multicolumn{2}{|c|}{ SIP68 Total } & \multicolumn{2}{|c|}{$\underline{\text { LLIP12 Total }}$} \\
\hline & $r$ & $p$ & $r$ & $p$ \\
\hline Age & 0.14 & .269 & 0.03 & .819 \\
\hline Male Gender & -0.05 & .719 & 0.02 & .861 \\
\hline $\begin{array}{l}\text { Non-White } \\
\text { Race/Ethnicity }\end{array}$ & $0.28 *$ & .030 & 0.21 & .099 \\
\hline Education & -0.23 & .080 & -0.12 & .353 \\
\hline
\end{tabular}

SIP68 = Sickness Impact Profile 68: Global Function

LLIP12 = Limb Loss Impact Profile 12: Global Function

*Significant at the 0.05 level 
Table 4.19 Correlations of SIP68 Physical Function with Demographic Variables

\begin{tabular}{|c|c|c|c|c|c|c|}
\hline \multirow[b]{2}{*}{ Variable } & \multicolumn{2}{|c|}{$\underline{\mathrm{SA}}$} & \multicolumn{2}{|c|}{$\underline{\mathrm{MC}}$} & \multicolumn{2}{|c|}{$\underline{\mathrm{MR}}$} \\
\hline & $r$ & $p$ & $\mathrm{r}$ & $p$ & $\mathrm{r}$ & $p$ \\
\hline Age & $0.26^{*}$ & .041 & $0.34 *$ & .008 & 0.09 & .514 \\
\hline Male Gender & -0.03 & .794 & -0.04 & .738 & -0.02 & 0872 \\
\hline $\begin{array}{l}\text { Non-White } \\
\text { Race/Ethnicity }\end{array}$ & $0.27 *$ & .039 & 0.17 & .195 & $0.28 *$ & .032 \\
\hline Education & $-0.28 *$ & 0.32 & -0.08 & .552 & -0.22 & .091 \\
\hline
\end{tabular}

SA = SIP68: Physical Function: Somatic Autonomy

$\mathrm{MC}=$ SIP68: Physical Function: Mobility Control

MR = SIP68: Physical Function: Mobility Range

*Significant at the 0.05 level 
Table 4.20 Correlations of SIP68 Psychosocial Function with Demographic Variables

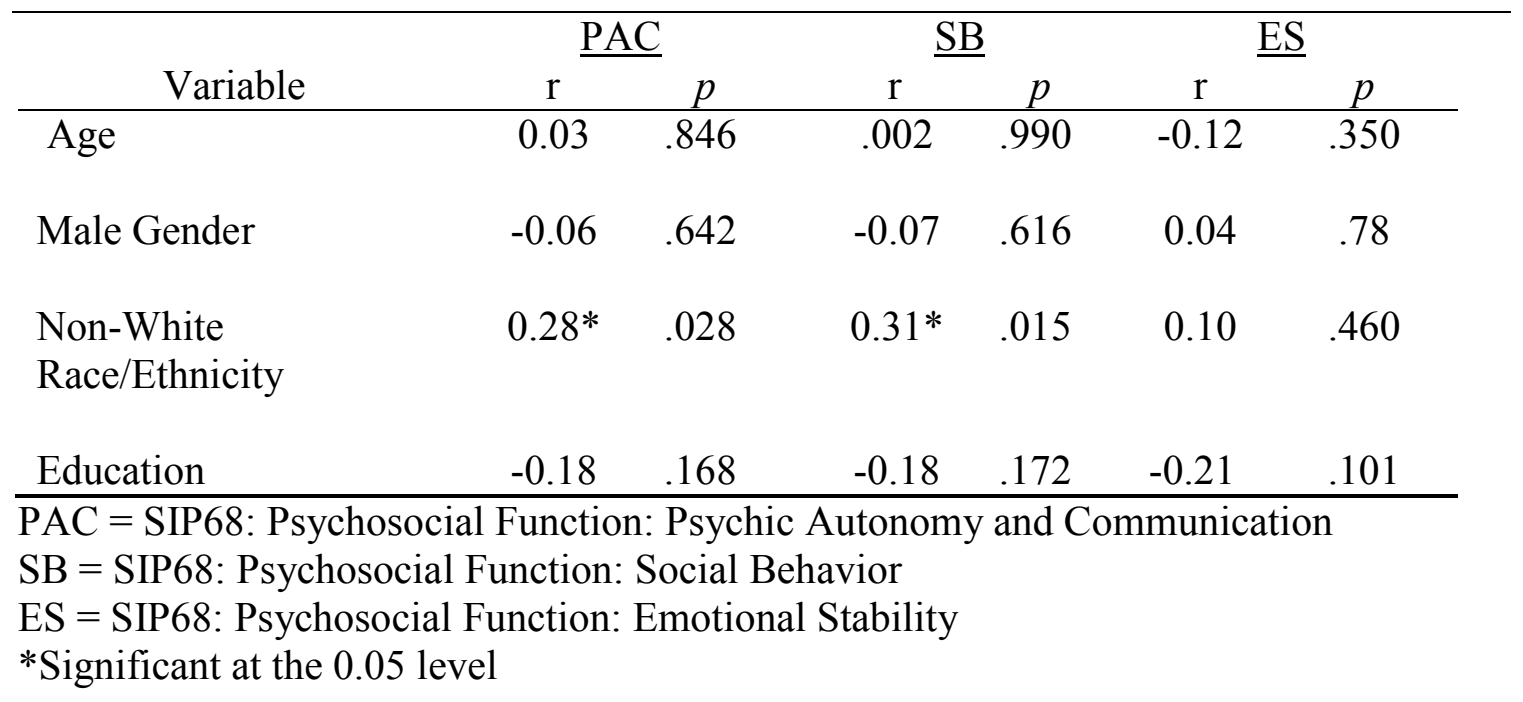


Table 4.21 Regression with Dependent Variable Global Function (SIP68 Total) $\left(\mathbf{R}^{2}=.52\right)$

Variable

Pain Intensity: Usual PLP

Pain Quality: Tight Band PLP

Non-White Race/Ethnicity

Pain Pattern: Continuous PLP

Amputation Type: Lower Extremity

Pain Quality: Tiring PLP

Pain Quality: Abnormal Position OP

Pain Pattern: Intermittent PLP

Pain Intensity: Usual OP

Pain Pattern: Intermittent OP

$\beta * *$

0.27

1.699

2.264

1.818

1.254

.216

0.16

0.14

1.316

.195

0.14

1.154

.254

0.14

1.123

.267

$-0.09$

$-.751$

.456

0.02

.158

.875

PLP $=$ Phantom Limb Pain

$\mathrm{OP}=$ Other Pain

*Significant at the 0.05 level

**Standardized Beta Coefficients 
Table 4.22 Regression with Dependent Variable Physical Function (Mobility Range) $\left(R^{2}=.51\right)$

\begin{tabular}{lccc}
\hline \multicolumn{1}{c}{ Variable } & $\beta^{* *}$ & $t$ & $p$ \\
\hline Pain Pattern: Continuous PLP & $0.29^{*}$ & 2.266 & .028 \\
Non-White Race/Ethnicity & $0.24^{*}$ & 2.124 & .039 \\
Pain Quality: Tight Band PLP & 0.22 & 1.913 & .062 \\
Pain Pattern: Intermittent OP & -0.22 & -1.713 & .093 \\
Pain Intensity: Usual PLP & 0.18 & 1.117 & .270 \\
$\begin{array}{l}\text { Amputation Type: Lower Extremity } \\
\text { Pain Quality: Tiring PLP }\end{array}$ & 0.16 & 1.399 & .169 \\
$\begin{array}{l}\text { Pain Quality: Abnormal Position OP } \\
\text { Pain Pattern: Intermittent PLP }\end{array}$ & 0.16 & 1.247 & .219 \\
$\begin{array}{l}\text { Pain Intensity: Usual OP } \\
\text { PLP = Phantom Limb Pain }\end{array}$ & 0.06 & .519 & .606 \\
$\begin{array}{l}\text { OP = Other Pain } \\
\text { * Significant at the 0.05 level }\end{array}$ & -0.05 & -.372 & .711 \\
& 0.04 & .251 & .803 \\
\hline
\end{tabular}




\section{Model to Predict Psychosocial Function (Social Behavior)}

In multiple linear regressions the candidate variables explained $46 \%$ of the variability in SIP68 psychosocial function: social behavior. Variables with the greatest magnitude in the model include usual PLP $(\beta=0.28, p=.101)$, Non-White race/ethnicity $(\beta=0.23, p=.051)$, tiring PLP $(\beta=0.18, p=.179)$, and abnormal position OP $(\beta=0.17, p=.185)$. Table 4.23 summarizes the results of the multiple regression. 
Table 4.23 Regression with Dependent Variable Psychosocial Function (Social Behavior) $\left(\mathrm{R}^{2}=.46\right)$

\begin{tabular}{lccc}
\hline \multicolumn{1}{c}{ Variable } & $\beta^{*}$ & $t$ & $p$ \\
\hline Pain Intensity: Usual PLP & 0.28 & 1.672 & .101 \\
Non-White Race/Ethnicity & 0.23 & 2.005 & .051 \\
Pain Quality: Tiring PLP & 0.18 & 1.365 & .179 \\
Pain Quality: Abnormal Position OP & 0.17 & 1.344 & .185 \\
$\begin{array}{l}\text { Pain Quality: Tight band PLP } \\
\text { Amputation Type: Lower Extremity }\end{array}$ & 0.16 & 1.306 & .198 \\
$\begin{array}{l}\text { Pain Pattern: Continuous PLP } \\
\text { Pain Intensity: Usual OP }\end{array}$ & 0.08 & .720 & .475 \\
Pain Pattern: Intermittent OP & 0.07 & .520 & .605 \\
Pain Pattern: Intermittent PLP & 0.05 & .301 & .765 \\
$\begin{array}{l}\text { PLP = Phantom Limb Pain } \\
\text { OP = Other Pain } \\
\text { * Standardized Beta Coefficients }\end{array}$ & -0.04 & -.319 & .751 \\
\end{tabular}




\section{CHAPTER 5: DISCUSSION}

\section{Introduction}

This discussion interprets the results and also compares and contrasts the results to current literature. The study's strengths and limitations are discussed, as well as directions for future research and clinical implications. The chapter concludes with a summary of key findings.

\section{Specific Aim 1}

Specific aim 1 was to describe the function, pain, health status, and demographics of community-dwelling, adult Mid-Southerners. The results are discussed below and are arranged by demographics, function, pain, and health status.

\section{Demographics}

\section{Age, Gender, and Race/Ethnicity}

This sample's mean age of 51.5 years was expected. The mean age was comparable to other community-based studies of function and pain in persons with limb loss for any cause that reported mean ages of 50.3, ${ }^{26} 53.9,{ }^{109} 54.0,{ }^{64} 58.1,{ }^{62} 61.2,{ }^{3}$ and 61.9. ${ }^{143}$ The mean age was higher than studies of community-dwelling persons with traumatic limb loss $(35.2 \pm 13.2),{ }^{4}$ and lower than studies of persons with vascular limb loss only $\left(65.9,{ }^{65} 68.0^{94,144}\right)$. Studies that have included only persons with vascular limb loss would be expected to have a sample mean of a higher age, since the risk for vascular limb loss increases with age. ${ }^{12}$ The sample was $65.6 \%$ male, which was less than expected, since male gender is a risk factor for limb loss. ${ }^{12}$ Percent male was comparable to studies on function in persons with limb loss, which were $67 \%$ male, ${ }^{109}$ $68 \%$ male $^{3}$ and $68.2 \%$ male. ${ }^{64}$ This study had a greater representation of women than most studies on function in persons with limb loss; those studies were $70.1 \%$ male, ${ }^{6}$ $73 \%,{ }^{145} 74 \%$ male, $77.1 \%$ male $,{ }^{6} 77.5 \%,{ }^{146} 78 \%$ male ${ }^{84,}{ }^{140}$ over $80 \%$ male, ${ }^{62} 83.8 \%$ male, ${ }^{103} 84 \%$ male, ${ }^{4} 87.7 \%$ male, ${ }^{65}$ and $100 \%$ male. $^{63,70}$ Thus, the ratio of male to female participants in this study is a unique contribution, because women are represented better than in previous studies. Additionally, most of the pain with limb loss studies have samples with a male majority. Previous studies reported $81 \%{ }^{23}$ and $100 \%,{ }^{27}$ and one of the classic surveys on pain and limb loss targeted military veterans who were all male. $^{80}$

White race/ethnicity was seen in $68.9 \%$ of the sample, with Blacks comprising $21.3 \%$ of the sample. Blacks are better represented in this study compared to other studies of function or pain with limb loss that were $4 \%$ Black, ${ }^{64} 5.4 \%$ Black, ${ }^{24} 5 \%$ Black, ${ }^{86,109} 5.4 \%$ Black, $^{24}$ and 7.2\% Black. ${ }^{26}$ Although the Black population in the 
South is the highest in the nation, Black race is a minority within the United States population. The Black population of the other Mid-South states is Alabama $26.4 \%$, Arkansas 15.8\%, Kentucky 7.3\%, Tennessee 16.8\% and Mississippi 36.3\%. ${ }^{147}$ Historically minorities tend to participate in research less than Whites, so this sample with an appropriate representation of Blacks is a unique contribution.

In summary, most studies of community-dwelling adults with limb loss have not had samples with adequate samples of Blacks or women. The percentage of Blacks and women in this study is a unique contribution that is important because of the health disparities associated with increased risk for both traumatic and vascular limb loss in Blacks and other Non-White minorities. ${ }^{12}$ There is also a gap in the literature describing function and pain in minority populations with limb loss. There is likewise a gap in the literature of how pain and function are related in women.

\section{Education}

This sample's mean education level was 13.6 years, with $18.0 \%$ who did not graduate from high school. This sample had a greater number of persons who did not graduate from high school compared to other studies of function and pain in communitydwelling adults with limb loss in the United States that reported $6.2 \%,{ }^{26} 12 \%,{ }^{64} 13 \%,{ }^{86}$ and $17 \%{ }^{109}$ of participants that did not graduate from high school. However, the sample was better educated than the Mid-South population with $24.1 \%-27.8 \%$ that did not graduate from high school. ${ }^{38}$ The recruitment of participants with low educational attainment for this study was difficult. Personal referrals from health care providers and support group coordinators provided recruitment of most limb loss participants with low educational attainment, because persons with low literacy are less likely to respond to written material or have internet access. Since education was chosen to represent socioeconomic status, there is sufficient variability in education level to represent a wide range in socioeconomic status.

\section{Function}

Global function (SIP68) was $19.7 \pm 14.5$, with a range of 1-54, which indicates that this population has impaired function. The SIP68 total results were comparable to previous studies of persons with limb loss by Streppel et al $(16.4)^{3}$ and Pernot et al (19.2). ${ }^{2}$ Streppel's sample was measured in the recovery phase, at two months post amputation surgery, and was drawn from a population of persons with limb loss in either outpatient or inpatient physical therapy programs. ${ }^{3}$ Pernot's study participants were also from the Netherlands and included data from persons with limb loss at one year post amputation. $^{2}$ Although these studies can't be compared directly as the health care resources and sample demographics are different from those living in the Mid-South, the data provide a reference point for discussion. The SIP136 was used to assess function in persons with traumatic limb loss in the United States and has shown that persons with limb loss showed impaired global function (SIP), with physical function impaired more than psychosocial function. 
Global function attributed specifically to limb loss (LLIP12 total) was $4.4 \pm 3.4$, with a range of $0-12$ out of a possible 12. The mean score for both measures of global function, the SIP68 total and the LLIP12 total, were about 1/3 of the total possible score, which is consistent with previous studies that reported the SIP68 total. This suggests that the impaired function in this sample can be attributed to limb loss specifically.

The mean scores for the SIP68 categories of physical function variables SA (2.6 \pm 3.0 [0-13]), MC (6.0 $\pm 3.5[0-12])$, and MR $(2.3 \pm 2.9$ [0-10]) and psychosocial variables PAC $(2.2 \pm 2.8[0-11]), \mathrm{SB}(4.9 \pm 3.9$ [0-11]), and ES (1.7 $\pm 1.7[0-6])$ were equivalent to Streppel. ${ }^{3}$ The standard deviation of SA and PAC show that there was much variability in the autonomy measures. Although global function was impaired in this sample, the autonomy variables SA and PAC were skewed suggesting that most of the sample functioned independently.

\section{Pain}

\section{Phantom Limb Pain}

The prevalence of PLP was $83.6 \%$, which was similar to other studies that reported $72 \%-85 \%$. $^{23,27,80}$ Pain intensity scores were converted from a verbal descriptor scale to a five point scale 0-4 for statistical analyses. The mean usual PLP $(1.1 \pm 0.9$ [03.5]) was found to be just above the lowest quartile and was slightly less than previous studies that reported average or usual PLP intensity was at about one-third ${ }^{27}$ to about one half $^{23,25,26}$ on a numeric $0-10$ scale. The mean worst PLP was found to be just above the mid-point $(2.5 \pm 1.4[0-4])$, which is consistent with a previous study which reported that worst PLP intensity was just above the mid-point ${ }^{27}$ and slightly lower than another study which reported that worst PLP was at the top quartile. ${ }^{25}$ The mean least pain intensity $(0.2 \pm 0.5[0-2])$ was just above no pain which is slightly lower than previous studies which reported that least PLP was at the lowest quartile. ${ }^{25}$ The mean current PLP $(0.6 \pm$ 0.8 [0-3]) was halfway into the first quartile and current PLP was half way to the midpoint, which suggests that the mean pain intensity at the time the test was administered was halfway between the least and usual PLP intensity. This suggests that most participants were not in pain or were experiencing less pain than usual at the time the test was administered.

The prevalence of PLP quality descriptors were burning 40.7\%, cramping 54.2\%, stabbing 59.3\%, shocking/shooting $61.0 \%$, tiring 33.9\%, twisting $20.3 \%$, abnormal position $35.6 \%$, and tight band $40.7 \%$. Previous studies that assessed for specific pain qualtity descriptors were similar burning (50\%), ${ }^{83}$ cramping (50\%), ${ }^{83}$ stabbing $\left(50 \%{ }^{83}\right.$, $72 \%{ }^{23}$ ), shooting $(76 \%)$, ${ }^{23}$ and tiring $(50 \%) .{ }^{83}$ A study that assessed for phantom limb pain quality descriptors by open ended questioning reported these same quality descriptors in much lower percentages - namely, hot 14\%-15\%, cramp 14\%-15\%, sharp shock/shooting 32\%-33\%, unusual position $4 \%$, and squeezing $13 \%-16 \%$. ${ }^{25}$ This demonstrates that the sensory pain quality descriptors assessed in this study were consistent with previous descriptors reported from persons not given specific prompts. 
Tiring, an affective pain quality descriptor provides information about the affective domain of the individual pain experience in persons with limb loss that was discovered in only one study that included eight persons with limb loss. ${ }^{83}$

Intermittent PLP (83.1\%) was more prevalent than continuous PLP (20.3\%). Previous studies reported phantom limb pain was intermittent or episodic, ${ }^{24,27,82}$ or both intermittent and constant. ${ }^{26}$ Since intermittent pain was more common than continuous pain in this sample, although both are represented, it can be concluded that the sample contains persons with and without chronic phantom limb pain.

\section{Residual Limb Pain}

The prevalence of residual limb pain was $55.0 \%$, less common than phantom limb pain, which is consistent with previous studies that reported a prevalence of $53 \%$ $76 \% .{ }^{23,24,79,84,85}$ The mean residual limb pain intensities, after converting them to a numeric value of $0-4$ were current RLP $(0.5 \pm 0.8$ [0-3]), halfway to the first quartile; usual RLP $(0.8 \pm 1.1[0-3])$, approaching the first quartile; worst RLP (1.7 \pm 1.7 [0-4]), approaching midpoint; and least RLP $(0.2 \pm 0.5$ [0-2]), approaching no pain. There were no studies found that reported residual limb pain intensities. The prevalence of RLP descriptors were burning $23.7 \%$, cramping $25.4 \%$, stabbing $32.2 \%$, shocking/ shooting $27.1 \%$, tiring $32.2 \%$, twisting $13.6 \%$, abnormal position $16.9 \%$, and tight band $22.0 \%$. There were no studies found that reported residual limb pain quality descriptors. Intermittent RLP (50.8\%) was more common than continuous RLP (13.6\%). Intermittent RLP was most common, a finding consistent with studies on residual limb pain in people with limb loss which reported intermittent RLP occurring in 45.0\%-65.1\% and continuous RLP occurring in $11.3 \%-23 \%{ }^{26,63}$

\section{Other Pain}

Positive report of OP was $62.7 \%$, which was consistent with studies that have reported back pain in $26.3 \%-76 \%$ of people with LE limb loss, ${ }^{24,26,87}$ as well as previous studies reporting pain in persons with upper extremity limb loss in the neck/upper back/contra-lateral shoulder in $45 \%$ of participants. ${ }^{62}$ The mean and standard deviation and range of OP intensities after converting them to a numeric value of $0-4$ were current OP $(0.7 \pm 0.9[0-3])$, approaching the lowest quartile; usual OP $(1.1 \pm 1.1[0-3])$ just over the lowest quartile, worst OP $(2.0 \pm 1.7$ [0-4]) at the mid-point, and least OP $(0.4 \pm 0.7$ [0-3]), approaching no pain. There were no studies found that reported the pain intensities of worst pain other than phantom pain or residual limb pain in persons with limb loss. The prevalence of OP quality descriptors were burning $25.4 \%$, cramping $30.5 \%$, stabbing $28.8 \%$, shocking/shooting $32.2 \%$, tiring $33.9 \%$, twisting $15.3 \%$, abnormal position $20.3 \%$, and tight band $10.2 \%$. Tiring, a pain quality descriptor from the affective domain of the McGill Pain Questionnaire, was the most common other pain quality descriptor, which exemplifies the affective impact of other pain in persons with limb loss. No studies were found that described other pain quality descriptors in persons with limb loss. Intermittent OP (50.8\%), was more common than continuous OP (28.8\%), which is consistent with previous studies of people with limb loss and back 
pain, which reported that back pain in people with limb loss experienced intermittent back pain (45\%-72\%) and continuous back pain $(17 \%-22 \%){ }^{26,86}$

The upper extremities were the most common pain site for other pain, with a total of $21.7 \%$ for all amputees. This was followed by the "sound" or intact contra-lateral limb, neck/back/shoulders, lower back, hips, buttocks, and superficial head pain. In a previous study, other common pain sites were back (17\%), neck/shoulders $(9 \%)$, and sound limb (7\%). ${ }^{23}$ This study concludes that pain in the upper extremities as well as neck/ upper back/shoulder area is common in persons with limb loss regardless of the type of limb loss being upper or lower extremity.

Pain in locations other than the phantom limb or residual limb may have many etiologies. There may be pain from an injury that occurred with the trauma that resulted in limb loss. Pain may be experienced in dysvascular limbs that are at risk for limb loss. Additionally, the stress on other areas of the body that occurs after limb loss can cause painful chronic conditions from overuse. The gait associated from ambulating with a prosthetic limb may result in back and joint pain. Persons wheelchair bound spend so much time sitting that it can result in pain at the back, hips, and buttocks. Using the upper extremities for transfers and for balance with ambulation can also result in chronic pain from overuse. The residual limb, hip or shoulder, and back can also become painful from the body mechanics of wearing a heavy prosthetic limb.

\section{Number of Pain Locations}

The mean of number of pain areas, which included all areas in pain, was $5.4 \pm$ $4.2(0-21)$ out of a possible 36 areas. This suggests that it is common to have more than one pain site in persons with limb loss which includes both intact body parts and missing limbs. There were no studies found that reported the number of pain areas with the location assessment method described by Escalante. ${ }^{132}$ However, studies have documented pain in more than one location and not just PLP and RLP. ${ }^{23,24,26,62,87}$

\section{Health Status}

\section{Diabetes, Smoking, Self-Assessment of Health, and Obesity}

The diabetes rate of $31.1 \%$ in the study population was about triple the expected Mid-South population's diabetes rates that ranged from $8.5 \%$ to $10.6 \%,{ }^{38}$ and includes the highest diabetes rates in the United States. This number was expected to be higher than the normal population since diabetes is a risk factor for limb loss. The prevalence of smoking now was $16.4 \%$ and ever smoked was $54.1 \%$. The rate of smoking was less than expected in the Mid-South general population and which ranged by state from $24.3 \%$ to $28.2 \%$. ${ }^{38}$ No previous studies that reported smoking rates of persons with limb loss were identified. The mean SAH approached the highest quartile of the scale and corresponds to good, the second highest rating on a five-point scale. Over half of the LEAP study participants with limb loss, a sample that was reported to have impaired 
global function, physical function, and psychosocial function, rated their health as excellent, which represented the best rating of health. ${ }^{61}$ The possibly over-rated selfassessment of health was consistent with previous studies that have reported that persons with limb rate their health higher than would be expected. ${ }^{65}$ Additionally, the general population of the persons living in the Delta region of Mississippi, representative of the Mid-South, was reported to rate their health higher than expected. ${ }^{148}$ The higher than expected SAH may have been due to cultural norms that include a low expectation of health status, especially with persons of middle to older aged. The BMI mean was 28.7, with an obesity rate of $37.7 \%$. The results were equivalent to studies that reported persons with limb loss had a mean BMI of $29.2,{ }^{6}$ and an obesity rate of $37 \% .{ }^{14}$ The height and weight used for the calculation of BMI were obtained by self-report, and no correction for lost body parts was made. The obesity rate was higher than the general population in the Mid-South, which was $27.4 \%$ to $32 \%,{ }^{38}$ and included the highest obesity rates in the United States. This study provides valuable information on an at risk regional population that leads the nation in rates of obesity, diabetes, smoking, and low educational attainment. The health disparities associated with this population place it at risk for limb loss and the associated pain and function impairment.

\section{Amputation History}

Most participants (77.0\%) were missing one limb, with $21.3 \%$ missing two limbs, and one participant was missing all four limbs. There was $11.9 \%$ of this sample who had upper extremity limb loss only which was expected, since lower extremity limb loss is more common than upper extremity limb loss. ${ }^{12}$ One large study with 539 participants, had comparable variability that compared function with limb loss type and reported that $14.7 \%$ of the male sample and $11.3 \%$ of the female sample had upper extremity limb loss only. ${ }^{103}$ Of this sample, $48 \%$ had lost either a knee or an elbow in at least one limb. The variability of the sample had an even representation of amputation levels. The amputation duration mean was about 8 years, suggesting that overall this sample is representative of persons with limb loss that have fully integrated into community life. The prevalence of traumatic cause of amputation was $45.9 \%$. This is slightly lower than other studies of community-dwelling persons with limb loss, where non-vascular or traumatic amputation was found in $47 \%-56 \%$ of the sample, ${ }^{110,143}$ which could be because of the high risk of vascular amputations in the Mid-South population. The lower than expected ratio of vascular amputation to might be because the comorbidities associated with vascular limb loss may necessitate nursing home placement so fewer persons with vascular limb loss live in the community. There was no studies found that reported the ratio of persons with limb loss who live in a nursing home versus the community. The Amputee Coalition of America's National Limb Loss Information Center was consulted and they reported that there were no known statistics on the ratio of persons living in a nursing home versus the community. With a lower number of persons with traumatic limb loss, it is expected that this sample would have fewer functional impairments than a sample that included more persons with vascular limb loss. 


\section{Specific Aim 2}

Specific aim two was to estimate the relationship between function and pain, health status, and demographics in community-dwelling, adult Mid-Southerners with limb loss. The discussion of the correlations of function and pain will be presented by the pain locations of phantom limb pain, residual limb pain, and other pain. Function is measured as global function, physical function, and psychosocial function. The results are described below.

\section{Correlations of Function and Pain}

\section{Correlation of Function with Phantom Limb Pain}

Positive report of experiencing PLP pain had a positive significant correlation with global function (SIP68 total and LLIP12 total), physical function (MC and MR), and psychosocial function (SB). These findings are consistent with previous research reporting phantom limb pain impairs global function, ${ }^{23,62,64,79}$ physical function, including observed decreased mobility ${ }^{79,90}$ and psychosocial function. ${ }^{26,85,91}$ These results were expected and demonstrate that reported phantom limb pain, independent of other pain variables, is disabling and impairs all domains of function.

Current PLP, usual PLP, and worst PLP had a positive significant correlation with global function (SIP68 total and LLIP12 total), physical function (MR), and psychosocial function (SB and ES). Usual PLP and worst PLP also had a positive correlation with SA and MC, and usual PLP was the only pain intensity variable related to PAC and therefore all of the function variables. Pearson's $r$ for the correlation of usual PLP with SIP68 total was very high and represented $38 \%$ of the shared variance. These findings are consistent with previous studies that reported average PLP intensity related to pain interference of activities of daily living, ${ }^{64}$ physical function of observed decreased mobility, ${ }^{90}$ and psychosocial function including bothersomeness. ${ }^{23,26,85,92}$ Usual PLP, which may represent chronic PLP, had a substantial relationship with all function variables. This provides strong support that the magnitude of phantom limb pain intensity, especially chronic phantom limb pain intensity, is closely related to all aspects of function.

Stabbing PLP, tiring PLP, and tight band PLP had positive significant correlations with global function (SIP68 total and LLIP12 total), physical function (MC), and psychosocial function (SB). Tiring PLP and tight band PLP also had a positive significant correlation with MR and PAC. Tight band PLP had the greatest magnitude of correlations of any of the PLP descriptors, and tight band PLP shared 19\% of the variance with LLIP12 total. There were no previous studies found that reported a relationship between these pain quality descriptors for PLP and global function, physical function, or psychosocial function. Tiring, which is an affective pain quality descriptor from the McGill Pain Questionnaire, ${ }^{78}$ suggests that pain description may capture more than just the physical description of pain; it may be more indicative of the individual 
experience of pain both physically and psychosocially. Pain quality descriptors of burning and cramping, which are classified as sensory descriptors by the McGill Pain Questionnaire, have been associated with psychophysiological etiologies of pain. ${ }^{81,82}$ Phantom limb pain quality descriptors provide a more complete, accurate pain assessment and shift PLP assessment to a more person-centered approach.

Intermittent PLP had a significant positive correlation with physical function (MC and MR). Continuous PLP had a significant positive correlation with global function (SIP68 total and LLIP12 total), physical function (SA, MC, and MR), and psychosocial function (SB and ES). Continuous PLP, similar to the pain intensity variable of usual PLP, is indicative of chronic pain, and had greater correlations with more function variables than intermittent PLP. Continuous PLP was more closely related to mobility range than mobility control and suggests that constant chronic pain was more closely related to where people went and what they did more than mobility capabilities.

\section{Correlation of Function with Residual Limb Pain}

Positive report of having any RLP was not correlated with any function variables. The lack of a relationship between having RLP and function might be that pain in the residual limb might be related to prosthetic limb use. A high level of function with a high activity level might lead to acute residual limb pain that was related to having no function impairment. Additionally, residual limb pain might just be a signal that an individual needs to have their prosthetic limb adjusted. There may also be persons with chronic residual limb pain that impairs function. The many scenarios of RLP etiology, which may be associated with either increased or decrease function may result in the lack of a correlation between RLP and function. This finding was not consistent with previous studies that reported that RLP was related to impaired global function, ${ }^{79,85}$ physical function with observed mobility, ${ }^{90}$ and RLP was related to degree of pain being bothersome. ${ }^{24,26}$ Except for observed mobility, these previous studies used instruments that included the assessment of pain or pain's influence, the relationship of RLP and function might be related to the instruments used. Since the SIP68 is a generic function instrument not related to pain or any specific pathology, this may explain why there was no relationship found here.

Usual RLP had a positive significant correlation with global function (LLIP12 total) and psychosocial function (ES). Chronic pain in the residual limb might cause more positive responses to the function assessment of limb loss for any reason, and may increase and therefore have an effect on emotional function. Current RLP also had a significant positive correlation with psychosocial function (ES), which may influence emotional state at the time of study participation. A previous study reported that RLP pain intensity was a greater predictor of pain interference than PLP or back pain, ${ }^{64}$ which was consistent with the correlation of usual RLP and current RLP to emotional stability. Since the LLIP12 total was the only global function measure that was related to usual RLP, it suggests that the relationships between residual limb pain variables with function may be attributed specifically to limb loss. 
Tight band RLP had a positive significant correlation with all function variables. Cramping RLP and abnormal position RLP had a positive significant correlation with global function (SIP68 total and LLIP12 total) and physical function (MC). Stabbing RLP had a positive significant correlation with global function (LLIP12 total) and physical function (MC). The correlation with the greatest magnitude of RLP descriptors and all function measures was tight band and global function (LLIP12 total), with a shared variance of 0.19 . Tight band RLP may be more closely related to impaired function than RLP intensities because it is more indicative of an etiology that results in pain-related impaired function. The effective descriptor of tiring was not associated with any function variables. This may be because RLP is expected, is less correlated with function, and therefore the affective domain of pain was not related to function. There were no previous studies found that reported a relationship between these pain quality descriptors for RLP and global function, physical function, or psychosocial function.

Intermittent RLP and continuous RLP were not related to any function variables. The lack of a relationship between residual limb pain pattern and function may be because of the many causes of residual limb pain, with no clear temporal pattern that is associated with impaired function. There were no studies found that reported the relationship between pain pattern RLP and function. The conclusion of this study is that intermittent RLP and continuous RLP did not have a relationship with function.

\section{Correlation of Function with Other Pain}

Positive report of other pain had a significant positive correlation with global function (LLIP12 total) only. This suggests that pain in locations other than the phantom limb and the residual limb is related specifically to impaired function attributed to limb loss. Previous studies reported that pain in locations other than PLP or RLP, including pain in the back or contra-lateral sound limb, was bothersome, ${ }^{23,24,64}$ and impaired physical function. ${ }^{86,93}$ This study's results can't be compared directly because participants' worst pain area was chosen to represent other pain, using the worst nonamputation-related pain may have strengthened the relationship between pain and function.

Current OP had a significant positive correlation with global function (SIP68 total and LLIP12 total), physical function (SA and MR), and psychosocial function (SB). Usual OP had a positive correlation with global function (SIP68 total and LLIP12 total), physical function (SA, MC, and MR), and psychosocial function (SB). This was consistent with previous work, which suggests pain intensity in places other than RLP or PLP was bothersome, ${ }^{24,26,86}$ with back pain related to impaired physical function ${ }^{86}$ and back pain intensity related to depressive symptoms. ${ }^{26}$ Since both current OP and usual OP had significant correlations with function, acute or chronic pain was related to impaired function. Since usual pain only was correlated with MC, this suggests that chronic other pain is more closely related to control over body movements.

Cramping OP, stabbing OP, tiring OP, and abnormal position had a positive significant correlation with global function (SIP68 total and LLIP12 total), twisting OP 
and stabbing OP with global function (LLIP12 total) only. Tiring OP and twisting OP had a positive significant correlation with physical function ( $\mathrm{MC}$ and $\mathrm{MR}$ ) and burning with physical function (SA). Cramping and abnormal position had a positive significant correlation with psychosocial function (SB and ES) and cramping with PAC, stabbing with SB, and tiring with ES. Since other pain may have been for any reason, the different pain quality descriptors may be associated with different underlying pain etiologies that affect different domains of function. There was no literature found that explored the relationship between other pain quality descriptors with global function.

Continuous OP had a positive significant correlation with global function (SIP68 total and LLIP12 total) and physical function (SA and MR). There were no studies found that address intermittent versus continuous pain in places other than phantom limb or the residual limb in persons with limb loss. Since only continuous OP was related to any function variables, this suggests that a pattern consistent with chronic pain is closely related to function.

\section{Correlation of Function with Number of Pain Areas}

Number of pain areas had a positive significant correlation with global function (SIP68 total and LLIP12 total), physical function (SA and MC), and psychosocial function (SB and ES). Persons with the greatest amount of pain areas have the greatest correlation with LLIP12, suggesting that the more pain areas an individual has the more participants attributed impaired function specifically to limb loss. There was $14 \%$ shared variance of number of pain areas with LLIP12. These results support the conclusion made by Marshall et al that suggested that pain in more than one site requires more than an assessment of pain intensity and should consider pain contributions of multiple pain sites. ${ }^{64}$ The conclusion from these findings is that the number of pain areas, which represents the total body area to have pain (including both intact and missing areas, was related to impaired function. The number of pain areas, which represents the total body area reported to have pain (including both intact and missing limbs), was related to the generic impairment of global function as well as global function attributed specifically to limb loss. The relationships between number of pain areas and SIP68 categories suggest that total area in pain may impair the ability to function independently without help or assistive devices as well as mobility control. However, the number of pain sites may not affect the need for assistance in performing behavioral tasks, but may interfere with relationships, and overall emotional status.

\section{Correlations of Function and Health Status}

\section{Correlation of Function with Diabetes, Smoking, Obesity, and Self-Assessment of Health}

Diabetes was not correlated with any function variables. Diabetes and limb loss are accompanied by co-morbidities associated with poorer physical function ${ }^{79}$ and psychosocial function, ${ }^{6}$ which often result in persons entering long term care and not 
returning to community life. ${ }^{7,95}$ The anticipated relationship between diabetes and impaired function may not be captured in this sample because this study only included community-dwelling persons with limb loss who may represent the highest end of the spectrum for function in persons with limb loss and diabetes. The only significant correlation with smoking now was positive correlation with psychosocial function (PAC) with $8 \%$ shared variance. Persons who currently smoked were more likely to have a higher PAC and therefore impaired mental functioning and communication. This impaired mental functioning may have inhibited smokers from making the decision to quit smoking, even though continuing to smoke was associated with increased health risks, including the loss of another limb. This result was consistent with previous studies that a relationship between impaired psychosocial function with smoking history ${ }^{4,5}$ in persons with limb loss.

There was no correlation between BMI and any function variables. These results are similar to a study that reported no relationship between obesity and function in persons with limb loss. ${ }^{97}$ SAH had a significant negative correlation with MC, control over one's movement. This is consistent with a study that reported walking distance was a predictor of subjective health related quality of life in lower limb amputees. ${ }^{79}$

\section{Correlation of Function with Amputation History}

Number of limbs lost had a significant positive correlation with physical function (SA), which was expected because the loss of more than one limb may create a greater need for physical support from others and assistive devices. Multiple limb loss did not influence mobility in this population, perhaps because the loss of at least one limb impacts mobility, or because of the inclusion of persons with upper extremity limb loss. Persons with bilateral lower limb loss have been reported to acquire the function level to lead normal lives, ${ }^{100}$ and with appropriate prosthesis devices persons with bilateral upper extremity limb loss are able to achieve a high level of function. ${ }^{101,102}$ Amputation type: lower extremity had significant correlation with physical function (MC and MR), which was consistent with a previous study that reported persons with lower extremity limb loss was related to greater physical disability. ${ }^{103}$ Although the mobility scores related to amputation type: lower extremity showed function impairment of mobility, the impairment of global function and psychosocial function in study that reported lower extremity limb loss was related to worse emotional reactions and decreased energy level but not to social isolation. These results are similar in that no relationship between amputation type and social behavior, but they differ in that there was no relationship was found between amputation type and emotional stability. These measures can't be directly compared, as they are based on different instruments, and the previous study was performed with a French sample.

Amputation level: AEA/AKA had no correlation with any function variables. The inclusion of persons with upper extremity limb loss may have influenced these results. These results are consistent with a study by Mackenzie et al that reported no difference related to above versus below the knee limb loss type using the SIP to measure function. ${ }^{4}$ However, this study reported that persons with below the knee limb 
loss had a greater walking speed. The SIP58 total and LLIP12 total may not measure within the range or may not have the sensitivity needed to detect the differences in mobility that have been reported in studies that involved observable measures. ${ }^{4,90}$ However, balance, which was an observed measure, was not related to amputation level in persons with lower extremity limb loss. ${ }^{104}$ The lack of a significant relationship between amputation level and function in this study may reflect that the subjective impact of function impairment was equivalent regardless of amputation level.

Amputation duration had a negative significant correlation with global function (SIP68 total and LLIP12 total), physical function (MC and MR), and psychosocial function (SB), suggesting that a longer time since amputation is associated with fewer functional impairments. Duration of amputation was not related to global function in people with limb loss who lost limbs as adults. ${ }^{4,5}$ However, amputation duration was related to better global function when limb loss occurred in childhood. ${ }^{103}$ This may be because limb loss in adulthood is more likely to have more associated co-morbidities. Additionally, there is a high rate a morbidity and mortality associated with medical limb loss, so that survivors with limb loss who live in the community represent a higher level of function than the limb loss population living in nursing homes. This relationship between amputation duration and global function in community-dwelling adults is complex and may not be fully described in this cross-sectional study.

Amputation cause: traumatic had a negative significant correlation with physical function (SA and $\mathrm{MC}$ ), as is consistent with a study that reported persons with traumatic limb loss performed better on observed physical mobility. ${ }^{107}$ The correlation with SA might be because persons with non-traumatic limb loss, which may be associated with greater co-morbidities secondary to diabetes, obesity, and smoking, therefore were more physically dependent on assistive devices and care givers.

\section{Correlations of Function and Demographics}

\section{Correlation of Function with Age, Gender, Non-White, and Race/Ethnicity}

Age had a significant positive correlation with physical function (SA and $\mathrm{MC}$ ); therefore, increasing age was associated with a greater level of dependency on others and assistive devices and decreased ability to control one's body. The results of the physical function variables of this study are consistent with previous studies that reported greater age was associated with decreased physical function in people with limb loss. ${ }^{7,60,90,103}$ Greater age was specifically associated with impaired mobility. ${ }^{\text {7, 60, 90,110 }}{ }^{\text {However, }}$ Molton et al reported that the relationship between age and pain interference diminished with increasing age. ${ }^{109}$ This may be because of the adaptive coping skills associated with aging. This complicated relationship may explain why no relationship was found between age and global function. The lack of a relationship between age and MR may explain why psychosocial function was not related to age. If mobility range was maintained, then psychosocial function would be maintained. Male gender was not related to any function variables. This contradicts most previous studies that reported 
that male gender was associated with better global function in people with limb loss. ${ }^{103,}$

110 However, these results were consistent with a study that report that gender was not a predictor of the psychosocial SIP. ${ }^{92}$

Non-White race/ethnicity had a significant positive correlation with global function (SIP68 total), physical function (SA and MR), and psychosocial function (PAC and SB). The greatest significant correlation was Non-White race/ethnicity with SB, which shared $10 \%$ of the variance. This was expected because in a study of Black and White chronic pain participants from the general population, Blacks showed a greater level of impairment in both the physical and psychosocial domains of the SIP136. ${ }^{129}$ Non-White race/ethnicity was related to the generic impairment of global function but not global function attributed specifically to limb loss, which suggests that function impairments are not specifically attributed to limb loss. These results were consistent with other studies that have reported impaired function related to Non-White race/ethnicity. ${ }^{4,5}$ Impaired function related to Non-White race/ethnicity may be the result of co-morbidities that may be related to dysvascular limb loss, as Non-White race/ethnicity is a risk factor for multiple cardiovascular morbidities. ${ }^{149,150}$ This would be expected in this sample in which over half have lost a leg secondary to non-traumatic causes. Since the Non-White race/ethnicities of Blacks, Latinos, and Native Americans have a higher limb loss risk, health disparities exist, so that not Non-White race/ethnicity is a risk for limb loss and for decreased function secondary co-morbidities associated with limb loss as well as impaired function from amputation.

\section{Correlation of Function with Education}

Education, which was chosen as a proxy for low socioeconomic status, had a negative significant correlation with physical function (SA). Therefore, with more education or a higher socioeconomic status, participants were more independent. This study did not achieve the significance to be consistent with previous studies that reported that low education was associated with impaired global function, physical function, and psychosocial function ${ }^{4,5,151}$ This also contradicts Mackenzie et al, who reported that education was more closely related to the psychosocial SIP than the physical SIP. ${ }^{4}$ 


\section{Specific Aim 3}

Specific aim 3 was to estimate the joint influences on function in persons with limb loss by pain, health status, and demographics living in community-dwelling, adult Mid-Southerners with limb loss. The analyses section in Chapter 3: Methods contains the details on how these candidate predictor variables were chosen. One variable from each function concept was chosen as an outcome variable for the models. One outcome variable from global function, physical function, and psychosocial function was chosen to represent outcomes in each function domain. The SIP68 total was chosen to represent global function, because it is a standardized measure of sickness-related dysfunction. Mobility range was chosen to represent physical function, because it is a measure of the influence of health status on performing usual tasks. Social behavior was chosen to represent psychosocial behavior, because it describes behavior of the relations with other people. The candidate predictor variables for all of the models were usual PLP, usual OP, tiring PLP, tight band PLP, abnormal position OP, intermittent PLP, continuous PLP, intermittent OP, amputation history type: lower extremity, and Non-White race/ethnicity.

\section{Model to Predict Global Function (SIP68 Total)}

In multiple linear regressions, these ten candidate variables explained $52 \%$ of the variance in global function (SIP68 total). The unique contribution to the variance of SIP68 total accounted for each individual predictor variable was usual PLP 27\%, tight band $26 \%$, Non-White race/ethnicity $20 \%$, continuous $16 \%$, amputation history type: lower extremity 14\%, tiring PLP 14\%, abnormal position OP 14\%, intermittent PLP 9\%, usual OP $2 \%$, and intermittent OP $0.2 \%$ of SIP 68 total variance.

Usual PLP accounted for the greatest amount of variance from SIP68 total. This is statistically consistent with the high correlations between usual PLP and SIP68 total. Usual PLP, which is suggestive of chronic phantom limb pain intensity, predicted the most variability of SIP68 total which demonstrates the impact of chronic phantom limb pain intensity on global function. Tight band PLP had the second greatest shared variability with SIP68 total, suggesting that specific descriptors have their own unique contribution to global function. Non-White race/ethnicity shared the third greatest magnitude of variability with SIP68 total, which exemplifies that the health disparities associated with Non-White race/ethnicity fall second only to pain variables. Non-White race/ethnicity was expected to predict the variability of global function because of the relationship between Non-White race/ethnicity and function. ${ }^{4,5}$ Continuous PLP shared the fourth greatest magnitude of variance, with SIP68 total demonstrating that continuous PLP explains more of the variance in SIP68 total than intermittent PLP or intermittent OP. This result, combined with the result for usual PLP, supports that greater chronic pain is closely related to impaired global function. The next greatest predictor of SIP68 total was amputation type: lower extremity, which was reported to be associated with greater impaired function, ${ }^{103}$ and was expected to be a one of the greatest predictors of global function in this model, especially since the SIP68 total contains two 
measures of mobility that were closely related to amputation type: lower extremity. The remaining predictors of SIP68 total were tiring PLP, abnormal position OP, intermittent PLP, usual OP, and Intermittent OP. The PLP variables were greater predictors of SIP68 total than the OP variables. Additionally, both intermittent PLP and intermittent OP which are measures of pain that may not be chronic were not as strong as the measures of usual PLP and continuous PLP, which are more suggestive of chronic pain.

\section{Model to Predict Physical Function (Mobility Range)}

In multiple linear regressions the candidate variables explained $51 \%$ of the variability of MR. Mobility range was chosen to represent physical function because it measures not just capabilities but a range of activities performed. The unique contribution to the variance of physical function (MR) accounted for by each individual predictor variable were continuous PLP, 29\%, Non-White race/ethnicity, 24\%, tight band PLP, 22\%, intermittent PLP -22\%, usual PLP18\%, amputation type: lower extremity $16 \%$, tiring PLP $16 \%$, abnormal position $6 \%$, intermittent PLP $-5 \%$, and usual OP $4 \%$.

Continuous PLP was the greatest predictor in this model, suggesting that the pain pattern is more predictive of physical function (MR) than a pain intensity variable.

Continuous PLP is consistent with unrelenting chronic phantom limb pain. With no relief or break from phantom limb pain, persons with limb loss do not travel far from home, regardless of their physical capabilities. Continuous PLP may also be predictive of isolation as the mobility range for where people go and what they actually do. NonWhite race/ethnicity was the second greatest predictor of MR, which was similar to the model for global function (SIP68 total). With a greater level of disability associated with Non-White race/ethnicity, these results were expected. With the close relationship demonstrated in this study of pain with function, the relationship of race/ethnicity with the variance of function remains a consistent predictor of both global function and physical function.

Tight band PLP was the next greatest predictor of physical function (MR). Similar to global function (SIP68 total), tight band was the pain quality descriptor that was the greatest predictor of physical function (MR). The correlation of intermittent PLP with physical function (MR) was positive, however, controlling for the other variables, and had a negative $\beta$ in this model. This reinforces how disabling to mobility range a constant pattern of phantom limb pain is, as the presence of constant pain is predictive of MR, but having time periods where phantom limb pain is absent is predictive of less impairment of MR. Additionally, controlling for continuous PLP, intermittent PLP became much less important in explaining the variance of MR. Usual PLP was the fifth greatest predictor of variance in MR, although usual PLP predicted the greatest amount of variance in both global function (SIP68 total) and psychosocial function (SB). This exemplifies that for mobility range, pain pattern is much more important than pain intensity because it predicted more of the variance of MR. Amputation type: lower extremity was the next greatest predictor of the variance in MR. 
It was surprising that amputation type: lower extremity was not the greatest predictor of variance in MR, since it was not expected that upper extremity limb loss would affect mobility. This may be because of the magnitude of the influence of pain variables, and race/ethnicity was a greater predictor of where people go and what they actually do than physical capabilities. Additionally, limb loss regardless of cause may accompany other factors such as co-morbidities and/or trauma-related injuries that impair mobility range. Tiring PLP predicted that next greatest variance of MR, which demonstrates the contribution of affective pain quality descriptors to where people go and what they do. Abnormal position OP predicted $6 \%$ of the variance in MR, which demonstrates the contribution of physical position pain quality descriptors. Usual OP predicted the least amount of variance of MR, 4\%. As stated earlier, with usual PLP, pain pattern was a greater predictor of MR than pain intensity.

\section{Model to Predict Psychosocial Function (Social Behavior)}

In multiple linear regressions the candidate variables explained $46 \%$ of the variability psychosocial function (SB). Social behavior is a measure of how much persons participate in social activities both in and outside of the home. The unique contribution to the variance of psychosocial function (SB) accounted for by each individual predictor variable was usual PLP 29\%, Non-White race/ethnicity 23\%, tiring PLP $18 \%$, abnormal position OP $17 \%$, tight band $16 \%$, amputation type, lower extremity $8 \%$, continuous PLP 7\%, usual OP 5\%, intermittent OP 4\%, and intermittent PLP 3\%.

Similar to the model for global function, usual PLP had the greatest contribution to SB. This is further evidence of how disabling the consequences of usual PLP are and the magnitude with which usual PLP affects social activities. Non-White race/ethnicity was the second greatest predictor of SB. This was the only candidate variable with a significant beta to predict SB. These results are consistent with the other two models, as Non-White race/ethnicity consistently explains a significant amount of variance in all function variables, and only pain variables were greater for all models. This suggests that although pain variables contribute significantly to the variance in function, NonWhite race/ethnicity is a consistent predictor, independent of pain, of function in community-dwelling adult Mid-Southerners with limb loss. The pain quality descriptors of tiring PLP, abnormal position OP, and tight band PLP, followed with similar betas, demonstrates that each pain quality descriptor had a unique contribution to SB. Amputation type: lower extremity explained much less of the variance of SB compared to the results of the model for MR. This would be expected, as impaired mobility is associated with lower extremity limb loss. Continuous PLP was the seventh greatest predictor of the variability of SB, which was surprising since continuous PLP was the greatest predictor of MR. This suggests that continuous pain in the phantom limb influences mobility range. Usual OP is the next greatest contributor to the variability of SB. The contribution of usual PLP is much greater than the contribution of usual OP in predicting variability of SB. This may be because of the many locations and etiologies associated with other pain. Intermittent OP and intermittent PLP both had a negative contribution to SB, controlling for the other variables in this model. Although other pain 
variables were predictive of the variance in SB, intermittent PLP and intermittent OP were not strong predictors of the variance in SB.

\section{Study Strengths}

\section{Specific Aim 1 Study Strengths}

This study had a greater percentage of Blacks as well as women than most previous studies on function and pain in persons with limb loss. The study recruited from many sources to reach persons with limb loss, including persons with low educational attainment, which might not be captured if they were recruited from one type of limb loss provider. This study included persons with both upper extremity and lower extremity limb loss.

Since it is common for persons in the Mid-South to speak in a Southern English dialect, ${ }^{130}$ the instrument used standardized, culturally appropriate prompts as necessary for psychometrically validated instruments as well as culturally appropriate authorgenerated items. By administering both the SIP68 total and the LLIP12 total, generic causes of impaired function could be compared to limb-loss-specific impaired function. The study instrument was administered verbally to accommodate persons with low educational attainment. A verbal descriptor scale for pain intensity was used to accommodate all levels of numeric comprehension. A verbal instrument provided the means to include persons with limb loss that might be missed in a written or electronic survey. Pain assessment was comprehensive and included pain location, pain intensity, pain quality descriptor, and pain pattern. This includes the affective pain quality descriptor, tiring. The results of this sample's function and pain measures are similar to previous studies, which suggest that the results are valid.

\section{Specific Aim 2 Study Strengths}

The correlation analyses results showed relationships that were closely related statistically. This may translate into clinically meaningful information that can be useful for clinicians and researchers. This includes the significant relationship between all pain variables and function. 


\section{Specific Aim 3 Study Strengths}

The magnitude of the multiple regressions suggests that the models were all strong models. This supports the meaningfulness of the independent variables in predicting function. Specifically, the pain variables consistent with chronic pain and the demographic variable, Non-White race/ethnicity were most predictive of function variability. Pain quality descriptors had a unique contribution to function variability; an original contribution to the literature as this relationship was not discovered to be previously reported.

\section{Study Limitations}

\section{Specific Aim 1 Study Limitations}

This is a cross-sectional study, so the experience of how function changes over time and the influence of pain, health status, and demographics over time was not captured. Since the study design was quantitative, the essence of the experience of limb loss may have been lost. All data was gathered by self-report, and subjective responses may not always be consistent with data in medical records, especially in persons with low health literacy. However, self- report does catch the subjective nature of function, pain, health status, and demographics. There was wide variability in individual amputation histories, including number of limbs loss, type of limb loss, level of amputation, duration of limb loss, and cause of limb loss, which threatens internal validity. However, all persons with limb loss share common experiences associated with limb loss population regardless of amputation history. Generalizability of this study is limited, as only persons from the Mid-South were included.

\section{Specific Aim 2 Study Limitations}

The number of correlations performed may have increased the chances for erroneous correlations to be found, a type I error. The theoretical and clinical significance of the correlation should be considered in interpreting the statistical significance. There are no previous studies that described the relationship between function and pain quality descriptors in persons with limb loss, which limits the ability to interpret the relationships.

Although there were relationships that were highly significant, the small sample size of 61 is a limitation. The a priori power analyses number of 84 was not achieved even with exhaustive recruitment methods so there may be subtle yet important relationships that were not detected. On the items that were not found to be significant, the findings may not fully explain all of the significant relationships, as there may be false negatives that do not reflect significant correlations, or a type II error. Very large sample sizes have been reported in a few studies of function in persons with limb loss 
with the following large sample sizes including soldiers from Sri Lanka $461,{ }^{63}$ persons with limb loss recruited nationally in the United States $478,{ }^{64}$ the LEAP project $601,{ }^{61}$ and French persons with upper or lower limb loss 539. ${ }^{103}$ However, there is precedence for a smaller sample size because many studies of function in persons limb loss that recruited locally included sample sizes of $29,{ }^{3} 30,{ }^{6} 46,{ }^{60} 60,{ }^{2}$ and $78 .{ }^{68}$ Additionally, the most recent study of pain in United States male soldiers with limb loss had a sample size of $30 .^{27}$

\section{Specific Aim 3 Study Limitations}

In the multiple regression models, although the independent variables were chosen for their individual contribution, it is inevitable that all pain variables have the potential to be inter-related. Steps were taken to eliminate collinear variables entered into the model (see analyses section, Chapter 3: Methods). However, each pain variable is unique, and the data for each pain variable was assessed individually, and each pain variable is a unique measure of pain. The sample size is a limitation for the generalization of the statistical models. Although, a post hoc power analyses showed that all models had a power of over 0.99 , the calculated post hoc power may be misleading as it is influenced by the size of the $p$ value and not sample size and does not predict of the probability of false negatives. ${ }^{152}$

\section{Directions for Future Research}

Future research is needed to describe the impact of upper versus lower extremity limb loss because the correlation of global function (SIP68 total) was very close to significant, and a study with greater statistical power and greater variability in limb loss type may yield more conclusive results. The research question regarding the difference in global function, physical function, and psychosocial function of persons with upper vs. lower limb loss should be investigated with a larger sample to include sufficient variability in both limb loss types.

Since this is the first study known to this author to investigate a statistical relationship between pain quality descriptors and function. Future research is needed to determine how the pain quality descriptors from the sensory and affective MPQ domains influence function. Assessment of pain quality descriptors should be done open ended first, then include prompts to determine the best method for obtaining pain quality descriptor data. The research question regarding the difference between the report of phantom limb pain quality descriptors when asked open ended versus given prompts could provide valuable information about phantom limb pain assessment. Additionally, if there are differences, do they influence the relationship between pain quality descriptors and function? The Correlation between phantom limb pain quality descriptors and function could be analyzed to determine the best method for obtaining information about the pain descriptions and how this influences the impairment of function. 
Since Non-White race/ethnicity was associated with impaired mobility range and social behavior, the risk for isolation in this population needs more specific research. A qualitative study to describe the lived experience of limb loss in minorities may provide insight to the correlation of Non-White race/ethnicity and impaired mobility range and social behavior. A culturally appropriate instrument to assess isolation would provide quantitative information on this phenomenon. Future studies that explore the impact of race/ethnicity on function in persons with limb loss need to recruit a larger sample size to increase the generalizability of the clinical implications.

The study of function in community-dwelling, adult Mid-Southerners with limb loss would be enriched by incorporating observed measures, as well as other subjective information such as report of falls and occupational status and family responsibilities. The research aim to describe observed function measures, number of falls, and occupational status correlate with subjective function instruments, in communitydwelling, adult Mid-Southerners with limb loss should be undertaken. Observed measures might include gait measures and the ability to perform tasks with upper extremities. The report of falls might be done with a personal log, combined with report from family members. Occupational status might include longitudinal studies of how the ability to adapt to limb loss in the work place and at home affects function.

Since the Mid-South is frequently referred to as the Bible Belt, religious beliefs and church association play a significant role in the lives of most Mid-Southerners, so research describing the role of religion in pain beliefs would provide insight into the relationship of religiosity and pain in community-dwelling, adult Mid-Southerners with limb loss. This could be done through standardized measures such as the God Locus of Health Control, which describes an individual's perception of how much God controls specific or general topics about their health. The research question, "What is the relationship of pain intensity and demographics with God Locus of Health Control in community-dwelling, adult Mid-Southerners with limb loss?" should be studied.

The prevention of non-traumatic limb loss should be studied in this at risk MidSouthern population. Culturally appropriate and affordable interventions that promote lower extremity preservation need to be investigated. The research question, "Does culturally appropriate patient teaching affect amputation outcome in Mid-Southerners at risk for dysvascular limb loss?" should be studied.

In summary, all future studies that assess pain and function in persons with limb loss need to be person centered and include a comprehensive assessment of pain, including location, intensity, pattern, and number of number of locations. It is also important to assess for all domains of function, as the impact of pain, health status, and demographics may be unique to a specific domain of function. Observable measures, qualitative study, and culturally appropriate measures would explain more about limb loss in Mid-Southerners. 


\section{Clinical Implications}

Mean PLP intensity variables and report of residual limb pain are similar to what was reported in previous studies published over the past 29 years, which suggests that the health care system needs to do more to prevent and treat phantom limb pain, residual limb pain, and other pain in persons with limb loss. The assessment of both function and pain in persons with limb loss requires a person centered approach, including determining how amputation affects all areas of function and all domains of pain including intensity, quality descriptors, and pattern. Prevention, assessment, and treatment of all pain, including neck, upper back, and upper extremity pain, needs to be a standard part of care plans for persons with limb loss, even after they have integrated into community life. The relationships between usual PLP and continuous PLP with function exemplify how important it is to prevent phantom limb pain from becoming severe or chronic to improve function outcomes. The correlations and multiple regression models showed that pain quality descriptors, including sensory and affective descriptors are associated with impaired function. Therefore, pain quality descriptors, including the affective descriptor tiring, should always be assessed in persons with limb loss. Persons with limb loss should be screened for isolation related to impaired mobility range and social behavior, with Non-White persons, especially Blacks, at greatest risk for isolation.

\section{Summary}

This descriptive, cross-sectional survey design study of community-dwelling adults with limb loss living in the Mid-South will be summarized here and organized by research question.

\section{Specific Aim 1}

This study recruited only from the Mid-South, an area of the United States that has the combination of demographics and health status that puts it at high risk for limb loss. Unique contributions of the demographics included a sample with representation of Non-White race/ethnicity (31.1\%), especially Blacks (21.3\%). Women were also well represented at $34.4 \%$. Although the education level was lower than in many studies, it was higher than the general Mid-South population. Function was similar to other studies of persons with limb loss. However, Blacks with chronic pain were shown to have a greater level of pain-related disability than Whites, ${ }^{31}$ and women tended to have a higher incidence of chronic pain ${ }^{153}$ and pain-related disability. ${ }^{154}$ Additionally Blacks showed a greater amount of impaired function than Whites in a previous study that measured function with the SIP136. ${ }^{129}$ This sample was expected to have a greater amount of pain and a greater amount of impaired function than previous studies, which was not what was found. 
Phantom limb pain and pain in places other than the phantom limb or residual limb were reported in most participants, and about half of the participant reported residual limb pain. The higher-than-expected level of function and lower-than-expected pain intensities demonstrate that although health disparities exist in the Mid-South, this sample had strengths to maintain function and coping skills for pain management. However, since the pain results are similar to phantom limb and residual limb pain studies done over the past three decades, there has been little progress in alleviating the frequency or severity of pain associated with limb loss.

Diabetes was reported in about one-third of the sample, and about one in six participants smoked. Participants rated their health as good, and about two-thirds of the sample were obese or overweight. About three quarters of the sample was missing one limb only, with most of the rest missing two limbs. Most of the sample had lower extremity limb loss only, and about one half were missing at least one elbow or knee. The average time since amputation was almost 9 years. About half the sample had traumatic limb loss.

\section{Specific Aim 2}

The report of phantom limb pain was related to impaired function; however, the phantom limb pain intensity variables usual PLP and worst PLP were most closely related to function. The pain phantom limb pain quality descriptors with close relationships to function were tiring PLP and tight band PLP. Continuous PLP was more closely related to impaired function than intermittent pain, suggesting that chronic pain was more disabling than intermittent pain. The report of residual limb pain was not related to function. However, usual RLP intensity was related to global function impaired by limb loss. The residual limb pain quality descriptors with close relationships to function were tiring RLP, abnormal position RLP and tight band RLP. Pain pattern was not related to RLP. The correlations of current OP and usual OP, suggestive of both acute and chronic pain at a site not associated with an amputation, impair function. Continuous OP was closely related to function, suggesting that chronic other pain was more closely associated with impaired function. A new finding was that correlations of pain quality descriptors in all locations suggest that the quality of pain with limb loss was related to function. The number of pain areas had a strong correlation with function. The correlations of all pain variables with function illustrate the need for a person-centered approach to pain assessment of all locations and the impact that it has on the daily life of persons with limb loss.

Current smoking was related to psychosocial dependence on others for daily activities. Self-assessment of health was related to mobility control only. The number of limbs loss was correlated with physical dependence on others or mechanical devices. Amputation type: lower extremity was related to mobility only. Amputation duration was not related to autonomy measures or emotional stability, but the other measures suggest that with a longer time since an amputation, there is less impaired function. Amputation cause and age were both related to impaired physical autonomy and 
impaired mobility control but not to mobility range, suggesting that the co-morbidities associated with a non-traumatic amputation and with advancing age may impair physical function. However, persons with non-traumatic limb loss and older age appear to have adapted to maintain mobility range. Non-White race/ethnicity was related to generically impaired global function but not limb loss specific global function, which is consistent with anticipated co-morbidities. Greater impairment of physical and psychosocial autonomy scores associated with Non-White race/ethnicity suggests a greater need for caregivers and assistive devices for persons with Non-White race/ethnicity. Mobility control was not related to Non-White race/ethnicity, but mobility range and social behavior suggests that Non-White race/ethnicity is a risk factor for isolation in persons with limb loss, regardless of physical mobility capabilities.

\section{Specific Aim 3}

The three multiple regression models predicted $46 \%-52 \%$ of the shared variance of global function, physical function, and psychosocial function. A previous study reported that average phantom limb pain, average residual limb pain, and average pack pain intensity predicted $20 \%$ of the variance in pain interference. ${ }^{64}$ These are much stronger models, which is evidence for including pain quality descriptors, and race/ethnicity into a prediction model of function.

Usual PLP explained the greatest amount of variance for both the SIP68 total and $\mathrm{SB}$, and continuous PLP explained the greatest amount of variance for MR. Usual PLP and continuous PLP each are suggestive of chronic pain yet have their own unique contribution. Since chronic severe phantom limb pain is difficult to treat, this can result in a cycle of increased pain coupled with increased disability that propagates so that the greater chronic phantom limb pain becomes, there is greater impairment of global function and social behavior. When phantom limb pain is constant and unrelenting, there is impairment of the range of activities performed.

Non-White race/ethnicity consistently explained the second or third greatest amount of variance in global function (SIP68 total), physical function (MR), and psychosocial function (SB). The health disparities associated with Non-White race/ethnicity consistently were predictive of function. This may be because of greater co-morbidities and a greater level of pain-related disability.

Most of the predictors were pain variables, and although pain variables are inherently inter-related, care was taken to find predictors that were not highly correlated, providing a unique contribution. The models support assessing for all pain variables to capture their unique contribution.

Amputation type: lower extremity did not have the expected magnitude to explain the function variables, especially MR. This demonstrates that pain can be more disabling than limb loss type, even for the range of activities performed. 


\section{LIST OF REFERENCES}

1. Ziegler-Graham K, MacKenzie EJ, Ephraim PL, Travison TG, Brookmeyer R. Estimating the prevalence of limb loss in the United States: 2005 to 2050. Archives of Physical Medicine \& Rehabilitation 2008;89:422-9.

2. Pernot HF, Winnubst GM, Cluitmans JJ, De Witte LP. Amputees in Limburg: incidence, morbidity and mortality, prosthetic supply, care utilisation and functional level after one year. Prosthetics \& Orthotics International 2000;24:906.

3. Streppel KR, de Vries J, van Harten WH. Functional status and prosthesis use in amputees, measured with the Prosthetic Profile of the Amputee (PPA) and the short version of the Sickness Impact Profile (SIP68). International Journal of Rehabilitation Research 2001;24:251-6.

4. MacKenzie EJ, Bosse MJ, Castillo RC, et al. Functional outcomes following trauma-related lower-extremity amputation. Journal of Bone \& Joint Surgery American Volume 2004;86-A:1636-45.

5. MacKenzie EJ, Bosse MJ, Pollak AN, et al. Long-term persistence of disability following severe lower-limb trauma. Results of a seven-year follow-up. Journal of Bone \& Joint Surgery - American Volume 2005;87:1801-9.

6. Peters EJ, Childs MR, Wunderlich RP, Harkless LB, Armstrong DG, Lavery LA. Functional status of persons with diabetes-related lower-extremity amputations. Diabetes Care 2001;24:1799-804.

7. Greive AC, Lankhorst GJ. Functional outcome of lower-limb amputees: a prospective descriptive study in a general hospital. Prosthetics \& Orthotics International 1996;20:79-87.

8. Marshall M, Helmes E, Deathe AB. A comparison of psychosocial functioning and personality in amputee and chronic pain populations. Clinical Journal of Pain 1992;8:351-7.

9. De Fretes A, Boonstra AM, Vos LD. Functional outcome of rehabilitated bilateral lower limb amputees. Prosthetics \& Orthotics International 1994;18:1824.

10. Smith JJ, Agel J, Swiontkowski MF, et al. Functional outcome of bilateral limb threatening: lower extremity injuries at two years postinjury. Journal of Orthopaedic Trauma 2005;19:249-53.

11. MacKenzie EJ, Jones AS, Bosse MJ, et al. Health-care costs associated with amputation or reconstruction of a limb-threatening injury. Journal of Bone \& Joint Surgery - American Volume 2007;89:1685-92.

12. Dillingham TR, Pezzin LE, MacKenzie EJ. Limb amputation and limb deficiency: epidemiology and recent trends in the United States. Southern Medical Journal 2002;95:875-83.

13. Ephraim PL, Dillingham TR, Sector M, et al. Epidemiology of limb loss and congenital limb deficiency: a review of the literature. Archives of Physical Medicine \& Rehabilitation 2003;84:747-61.

14. Kurdibaylo SF. Obesity and metabolic disorders in adults with lower limb amputation. Journal of Rehabilitation Research \& Development 1996;33:387-94. 
15. Zeltsman D, Kerstein MD. Sociology of care in patients with severe peripheral vascular disease. American Surgeon 1998;64:175-7.

16. Wachtel MS. Family poverty accounts for differences in lower-extremity amputation rates of minorities 50 years old or more with diabetes. Journal of the National Medical Association 2005;97:334-8.

17. Boyle JP, Honeycutt AA, Narayan KM, et al. Projection of diabetes burden through 2050: impact of changing demography and disease prevalence in the U.S. Diabetes Care 2001;24:1936-40.

18. U. S. Obesity Trends 1985-2007. Centers for Disease Control and Prevention, 2007. (Accessed December 2, 2008, at http://www.cdc.gov/nccdphp/dnpa/obesity/trend/maps/index.htm.)

19. Centers for Disease Control and Prevention. Public Health and Aging: Trends in Aging - United States and Worldwide. In: Morbidity and Mortality Weekly Report; 2003:101-6.

20. Population Projections for States by Age, Sex, Race, and Hispanic Origin: 1995 to 2025. Population Projections Branch Population Division. U.S. Bureau of the Census, 1996. (Accessed July 30, 2009, at http://www.census.gov/population/www/projections/ppl47.html.)

21. Centers for Disease Control and Prevention. Cigarette Smoking Among AdultsUnited States, 2007. In: Morbidity and Mortality Weekly Report CDC; 2008:1221-6.

22. DeNavas-Walt C, Proctor, BD and Smith, JC. Income, Poverty, and Health Insurance Coverage in the United States: 2007. In: Washington, DC: U.S. Census Bureau, U.S. Government Printing Office; 2008.

23. Ehde DM, Czerniecki JM, Smith DG, et al. Chronic phantom sensations, phantom pain, residual limb pain, and other regional pain after lower limb amputation. Archives of Physical Medicine \& Rehabilitation 2000;81:1039-44.

24. Smith DG, Ehde DM, Legro MW, Reiber GE, del Aguila M, Boone DA. Phantom limb, residual limb, and back pain after lower extremity amputations. Clinical Orthopaedics \& Related Research 1999:29-38.

25. Sherman RA, Sherman CJ. A comparison of phantom sensations among amputees whose amputations were of civilian and military origins. Pain 1985;21:91-7.

26. Ephraim PL, Wegener ST, MacKenzie EJ, Dillingham TR, Pezzin LE. Phantom pain, residual limb pain, and back pain in amputees: results of a national survey. Archives of Physical Medicine \& Rehabilitation 2005;86:1910-9.

27. Ketz AK. The experience of phantom limb pain in patients with combat-related traumatic amputations. Archives of Physical Medicine \& Rehabilitation 2008;89:1127-32.

28. Whyte A, Niven CA. The illusive phantom: does primary care meet patient need following limb loss? Disability \& Rehabilitation 2004;26:894-900.

29. Chen I, Kurz J, Pasanen M, et al. Racial differences in opioid use for chronic nonmalignant pain. Journal of General Internal Medicine 2005;20:593-8.

30. Ezenwa MO, Ameringer S, Ward SE, Serlin RC. Racial and ethnic disparities in pain management in the United States. Journal of Nursing Scholarship 2006;38:225-33. 
31. Green CR, Baker TA, Sato Y, Washington TL, Smith EM. Race and chronic pain: A comparative study of young black and white Americans presenting for management. Journal of Pain 2003;4:176-83.

32. Fuentes M, Hart-Johnson T, Green CR. The association among neighborhood socioeconomic status, race and chronic pain in black and white older adults. Journal of the National Medical Association 2007;99:1160-9.

33. Whyte AS, Carroll LJ. A preliminary examination of the relationship between employment, pain and disability in an amputee population. Disability \& Rehabilitation 2002;24:462-70.

34. Wolf MS, Gazmararian JA, Baker DW. Health literacy and functional health status among older adults. Archives of Internal Medicine 2005;165:1946-52.

35. Sherman RA. Phantom Pain. New York: Plenum Press; 1997.

36. Huse E, Larbig W, Flor H, Birbaumer N. The effect of opioids on phantom limb pain and cortical reorganization. Pain 2001;90:47-55.

37. Bloomquist T. Amputation and phantom limb pain: a pain-prevention model. American Association of Nurse Anethnesists Journal 2001;69:211-7.

38. Shortchanging America's Health 2008. (Accessed January 20, 2009 at http://healthyamericans.org/reports/shortchanging08/.)

39. Bishaw A. Areas With Concentrated Poverty: 1999 In: Census 2000 Special Reports: U.S. Department of Commerce Economics and Statistics Administration US Census Bureau2005.

40. Reich JW, Olmsted ME, van Puymbroeck CM. Illness uncertainty, partner caregiver burden and support, and relationship satisfaction in fibromyalgia and osteoarthritis patients. Arthritis \& Rheumatism 2006;55:86-93.

41. The Physical Therapy Dictionary. 2009. (Accessed January 13, 2009, at $\leq$ http://www.physicaltherapydictionary.com/F.aspx?Term=function $>$ )

42. International Association for the Study of Pain Terminology. (Accessed July 30, 2009, at http://www.iasp-

pain.org/AM/Template.cfm?Section=Pain_Definitions\&Template=/CM/HTMLD isplay.cfm\&ContentID=1728\#Pain.)

43. Exploring the Complexity of Advocacy: Balancing Patient-Centered Care and Safety. 2007. (Accessed August18, 2009, at $\leq$ http://www.qsen.org/teachingstrategy.php?id=58>)

44. Dictionary.com. (Accessed October 13, 2009, at http://dictionary.reference.com/browse/action.)

45. Robinson JP, Turk DC, Loeser JD. Pain, impairment, and disability in the AMA guides. Journal of Law, Medicine \& Ethics;32:315-26.

46. Lawton MP. The functional assessment of elderly people. Journal of the American Geriatrics Society 1971;19:465-81.

47. Dictionary.com. (Accessed August 13, 2009, at http://dictionary.reference.com/browse/task.)

48. Beginnings of Physiology in the US (1820-1892). (Accessed November 15, 2006, at http://www.physiology.arizona.edu/index.php/articles/560.)

49. Shultz D. A History of Modern Psychology. 3rd ed. New York: Academic Press; 1981. 
50. James McKeen Cattell. 1997. (Accessed November 19, 2006, at http://www.muskingum.edu/ psych/psycweb/history/cattell.htm\#Theory.)

51. Gaitz CM, Baer PE. Diagnostic assessment of the elderly: a multifunctional model. Gerontologist 1970;10:47-52.

52. Lawton MP. Assessment, integration, and environments for older people. Gerontologist 1970;10:38-46.

53. Bloom M, Blenkner M. Assessing functioning of older persons living in the community. Gerontologist 1970;10:31-7.

54. Katz S, Downs TD, Cash HR, Grotz RC. Progress in development of the index of ADL. Gerontologist 1970;10:20-30.

55. Patston P. Constructive Functional Diversity: a new paradigm beyond disability and impairment. Disability \& Rehabilitation 2007;29:1625-33.

56. Dictionary.com. (Accessed October 14, 2009, at http://dictionary.reference.com/browse/norm.)

57. Rondinelli RD, Katz RT. Merits and shortcomings of the American Medical Association Guides to the Evaluation of Permanent Impairment, 5th edition. A physiatric perspective. Physical Medicine \& Rehabilitation Clinics of North America; 13:355-70.

58. Bergner M, Bobbitt RA, Kressel S, Pollard WE, Gilson BS, Morris JR. The sickness impact profile: conceptual formulation and methodology for the development of a health status measure. International Journal of Health Services 1976;6:393-415.

59. Dictionary.com. (Accessed October 13, 2009, at http://dictionary.reference.com/browse/role.)

60. Schoppen T, Boonstra A, Groothoff JW, de Vries J, Goeken LN, Eisma WH. Physical, mental, and social predictors of functional outcome in unilateral lowerlimb amputees. Archives of Physical Medicine \& Rehabilitation 2003;84:803-11.

61. MacKenzie EJ, Bosse MJ, Kellam JF, et al. Characterization of patients with high-energy lower extremity trauma. Journal of Orthopaedic Trauma 2000;14:455-66.

62. Datta D, Selvarajah K, Davey N. Functional outcome of patients with proximal upper limb deficiency--acquired and congenital. Clinical Rehabilitation 2004; 18:172-7.

63. Gunawardena NS, Seneviratne Rde A, Athauda T. Functional outcomes of unilateral lower limb amputee soldiers in two districts of Sri Lanka. Military Medicine 2006;171:283-7.

64. Marshall HM, Jensen MP, Ehde DM, Campbell KM. Pain site and impairment in individuals with amputation pain. Archives of Physical Medicine \& Rehabilitation 2002;83:1116-9.

65. Harness N, Pinzur MS. Health related quality of life in patients with dysvascular transtibial amputation. Clinical Orthopaedics \& Related Research 2001;383:2047.

66. Smith DG, Horn P, Malchow D, Boone DA, Reiber GE, Hansen ST, Jr. Prosthetic history, prosthetic charges, and functional outcome of the isolated, traumatic below-knee amputee. Journal of Trauma-Injury Infection \& Critical Care 1995;38:44-7. 
67. de Bruin AF, Buys M, de Witte LP, Diederiks JP. The sickness impact profile: SIP68, a short generic version. First evaluation of the reliability and reproducibility. Journal of Clinical Epidemiology 1994;47:863-71.

68. Pezzin LE, Dillingham TR, MacKenzie EJ. Rehabilitation and the long-term outcomes of persons with trauma-related amputations. Archives of Physical Medicine \& Rehabilitation 2000;81:292-300.

69. Wexler DJ, Grant RW, Wittenberg E, et al. Correlates of health-related quality of life in type 2 diabetes. Diabetologia 2006;49:1489-97.

70. Dougherty PJ. Long-term follow-up of unilateral transfemoral amputees from the Vietnam war. Journal of Trauma-Injury Infection \& Critical Care 2003;54:71823.

71. Dougherty PJ. Transtibial amputees from the Vietnam War. Twenty-eight-year follow-up. Journal of Bone \& Joint Surgery - American Volume 2001;83-A:3839.

72. Meldrum ML. A capsule history of pain management. Journal of the American Medical Association 2003;290:2470-5.

73. Unruh AM. Spirituality, religion, and pain. Canadian Journal of Nursing Research 2007;39:66-86.

74. O'Rourke K. Pain relief: the perspective of Catholic tradition. Journal of Pain \& Symptom Management 1992;7:485-91.

75. Livingston W. Pain and Suffering. Seatle: International Association for the Study of Pain Press; 1998.

76. Melzack R, Wall PD. Pain mechanisms: a new theory. Science 1965;150:971-9.

77. Mccaffery M. Nursing practice theories related to cognition, bodily pain, and man-environment interactions. Los Angeles: University of California Los Angeles Students' Store; 1968.

78. Melzack R. The McGill Pain Questionnaire: major properties and scoring methods. Pain 1975;1:277-99.

79. van der Schans CP, Geertzen JH, Schoppen T, Dijkstra PU. Phantom pain and health-related quality of life in lower limb amputees. Journal of Pain \& Symptom Management 2002;24:429-36.

80. Sherman RA, Sherman CJ, Parker L. Chronic phantom and stump pain among American veterans: results of a survey. Pain 1984;18:83-95.

81. Sherman RA, Bruno GM. Concurrent variation of burning phantom limb and stump pain with near surface blood flow in the stump. Orthopedics 1987;10:1395-402.

82. Sherman RA, Griffin VD, Evans CB, Grana AS. Temporal relationships between changes in phantom limb pain intensity and changes in surface electromyogram of the residual limb. International Journal of Psychophysiology 1992;13:71-7.

83. Dubuisson D, Melzack R. Classification of clinical pain descriptions by multiple group discriminant analysis. Experimental Neurology 1976;51:480-7.

84. Graham L, Parke RC, Paterson MC, Stevenson M. A study of the physical rehabilitation and psychological state of patients who sustained limb loss as a result of terrorist activity in Northern Ireland 1969-2003. Disability \& Rehabilitation 2006;28:797-801. 
85. Gallagher P, Allen D, Maclachlan M. Phantom limb pain and residual limb pain following lower limb amputation: a descriptive analysis. Disability \& Rehabilitation 2001;23:522-30.

86. Ehde DM, Smith DG, Czerniecki JM, Campbell KM, Malchow DM, Robinson LR. Back pain as a secondary disability in persons with lower limb amputations. Archives of Physical Medicine \& Rehabilitation 2001;82:731-4.

87. Stam HJ, Dommisse AM, Bussmann HJ. Prevalence of low back pain after transfemoral amputation related to physical activity and other prosthesis-related parameters. Disability \& Rehabilitation 2004;26:794-7.

88. Norvell DC, Czerniecki JM, Reiber GE, Maynard C, Pecoraro JA, Weiss NS. The prevalence of knee pain and symptomatic knee osteoarthritis among veteran traumatic amputees and nonamputees. Archives of Physical Medicine \& Rehabilitation 2005;86:487-93.

89. Friel K, Domholdt E, Smith DG. Physical and functional measures related to low back pain in individuals with lower-limb amputation: an exploratory pilot study. Journal of Rehabilitation Research \& Development 2005;42:155-66.

90. Geertzen JH, Bosmans JC, van der Schans CP, Dijkstra PU. Claimed walking distance of lower limb amputees. Disability \& Rehabilitation 2005;27:101-4.

91. Arena JG, Sherman RA, Bruno GM, Smith JD. The relationship between situational stress and phantom limb pain: cross-lagged correlational data from six month pain logs. Journal of Psychosomatic Research 1990;34:71-7.

92. Hanley MA, Jensen MP, Ehde DM, Hoffman AJ, Patterson DR, Robinson LR. Psychosocial predictors of long-term adjustment to lower-limb amputation and phantom limb pain. Disability \& Rehabilitation 2004;26:882-93.

93. Kulkarni J, Gaine WJ, Buckley JG, Rankine JJ, Adams J. Chronic low back pain in traumatic lower limb amputees. Clinical Rehabilitation 2005;19:81-6.

94. Kazmers A, Perkins AJ, Jacobs LA. Major lower extremity amputation in Veterans Affairs medical centers. Annals of Vascular Surgery 2000;14:216-22.

95. De Luccia N, Pinto MA, Guedes JP, Albers MT. Rehabilitation after amputation for vascular disease: a follow-up study. Prosthetics \& Orthotics International 1992;16:124-8.

96. Aulivola B, Hile CN, Hamdan AD, et al. Major lower extremity amputation: outcome of a modern series. Archives of Surgery 2004;139:395-9; discussion 9.

97. Kalbaugh CA, Taylor SM, Kalbaugh BA, et al. Does obesity predict functional outcome in the dysvascular amputee? American Surgeon 2006;72:707-12; discussion 12-3.

98. Wilcox VL, Kasl SV, Idler EL. Self-rated health and physical disability in elderly survivors of a major medical event. Journals of Gerontology Series BPsychological Sciences \& Social Sciences 1996;51:S96-104.

99. Miller WC, Deathe AB. A prospective study examining balance confidence among individuals with lower limb amputation. Disability \& Rehabilitation 2004;26:875-81.

100. Dougherty PJ. Long-term follow-up study of bilateral above-the-knee amputees from the Vietnam War. Journal of Bone \& Joint Surgery - American Volume 1999;81:1384-90. 
101. Johansen PB, Breitholtz M, Cavrini R, Johansson B, Schmidl H, Thyberg M. Prosthetic rehabilitation in bilateral high above elbow amputation. Scandinavian Journal of Rehabilitation Medicine 1987;19:85-7.

102. Hung JW, Wu YH. Fitting a bilateral transhumeral amputee with utensil prostheses and their functional assessment 10 years later: a case report. Archives of Physical Medicine \& Rehabilitation 2005;86:2211-3.

103. Demet K, Martinet N, Guillemin F, Paysant J, Andre JM. Health related quality of life and related factors in 539 persons with amputation of upper and lower limb. Disability \& Rehabilitation 2003;25:480-6.

104. Miller WC, Deathe AB, Speechley M. Psychometric properties of the Activitiesspecific Balance Confidence Scale among individuals with a lower-limb amputation. Archives of Physical Medicine \& Rehabilitation 2003;84:656-61.

105. Huang CT, Jackson JR, Moore NB, et al. Amputation: energy cost of ambulation. Archives of Physical Medicine \& Rehabilitation 1979;60:18-24.

106. Melchiorre PJ, Findley T, Boda W. Functional outcome and comorbidity indexes in the rehabilitation of the traumatic versus the vascular unilateral lower limb amputee. American Journal of Physical Medicine \& Rehabilitation 1996;75:9-14.

107. Torburn L, Powers CM, Guiterrez R, Perry J. Energy expenditure during ambulation in dysvascular and traumatic below-knee amputees: a comparison of five prosthetic feet. Journal of Rehabilitation Research \& Development 1995;32:111-9.

108. Nagarajan R, Clohisy DR, Neglia JP, et al. Function and quality-of-life of survivors of pelvic and lower extremity osteosarcoma and Ewing's sarcoma: the Childhood Cancer Survivor Study. British Journal of Cancer 2004;91:1858-65.

109. Molton IR, Jensen MP, Ehde DM, Smith DG. Phantom limb pain and pain interference in adults with lower extremity amputation: The moderating effects of age. Rehabilitation Psychology 2007;52:272-9.

110. Miller WC, Deathe AB, Harris J. Measurement properties of the Frenchay Activities Index among individuals with a lower limb amputation. Clinical Rehabilitation 2004; 18:414-22.

111. Gailey RS, Roach KE, Applegate EB, et al. The amputee mobility predictor: an instrument to assess determinants of the lower-limb amputee's ability to ambulate. Archives of Physical Medicine \& Rehabilitation 2002;83:613-27.

112. Taylor SM, Kalbaugh CA, Blackhurst DW, et al. Preoperative clinical factors predict postoperative functional outcomes after major lower limb amputation: an analysis of 553 consecutive patients. Journal of Vascular Surgery 2005;42:22735.

113. National Institure for Literacy. The State of Literacy in America: Estimates at the local, state, and national levels. Washington, DC: National Institure for Literacy; 1998.

114. Machin PB, de C. Williams AC. Stiff upper lip: coping strategies of world war II veterans with phantom limb pain. Clinical Journal of Pain 1998;14:290-4.

115. Franchignoni F, Orlandini D, Ferriero G, Moscato TA. Reliability, validity, and responsiveness of the locomotor capabilities index in adults with lower-limb amputation undergoing prosthetic training. Archives of Physical Medicine \& Rehabilitation 2004;85:743-8. 
116. Lehman CM, Clark HB, Bullis M, Rinkin J, Castellanos LA. Transition from school to adult life: empowering youth through community ownership and accountability. Journal of Child and Family Studies 2002;11:127-41.

117. Jensen MP, Smith DG, Ehde DM, Robinsin LR. Pain site and the effects of amputation pain: further clarification of the meaning of mild, moderate, and severe pain. Pain 2001;91:317-22.

118. Ketz AK. Pain management in the traumatic amputee. Critical Care Nursing Clinics of North America 2008;20:51-7.

119. Turk DC, Melzack R. Handbook of Pain Assessment. 2nd ed. New York: Guilford Press; 2001.

120. Schoppen T, Boonstra A, Groothoff JW, van Sonderen E, Goeken LN, Eisma WH. Factors related to successful job reintegration of people with a lower limb amputation. Archives of Physical Medicine \& Rehabilitation 2001;82:1425-31.

121. Gilson B, Bergner M, Bobbit R, Carter W. The SIP: Final development and testing, 1975-1978. Seatle: Department of Community Medicine, University of Washington; 1978.

122. Gilson BS, Gilson JS, Bergner M, et al. The sickness impact profile. Development of an outcome measure of health care. American Journal of Public Health 1975;65:1304-10.

123. Bergner M, Bobbitt RA, Carter WB, Gilson BS. The Sickness Impact Profile: development and final revision of a health status measure. Medical Care 1981;19:787-805.

124. Grise MC, Gauthier-Gagnon C, Martineau GG. Prosthetic profile of people with lower extremity amputation: conception and design of a follow-up questionnaire. Archives of Physical Medicine \& Rehabilitation 1993;74:862-70.

125. de Bruin AF, Diederiks JP, de Witte LP, Stevens FC, Philipsen H. The development of a short generic version of the Sickness Impact Profile. Journal of Clinical Epidemiology 1994;47:407-18.

126. Post MW, Gerritsen J, Diederikst JP, DeWittet LP. Measuring health status of people who are wheelchair-dependent: validity of the Sickness Impact Profile 68 and the Nottingham Health Profile. Disability \& Rehabilitation 2001;23:245-53.

127. Nanda U, McLendon PM, Andresen EM, Armbrecht E. The SIP68: an abbreviated sickness impact profile for disability outcomes research. Quality of Life Research 2003;12:583-95.

128. Post MW, de Bruin A, de Witte L, Schrijvers A. The SIP68: a measure of healthrelated functional status in rehabilitation medicine. Archives of Physical Medicine \& Rehabilitation 1996;77:440-5.

129. Cano A, Mayo A, Ventimiglia M. Coping, pain severity, interference, and disability: the potential mediating and moderating roles of race and education. Journal of Pain 2006;7:459-68.

130. Lobov W, Ash S, Boberg C. The Atlas of North American English. Berlin: Mouton-de Gruyter; 2006.

131. Sherman RA. Pain Assessment and Intervention from a Psychophysiological Perspective. Wheat Ridge, CO: Association for Applied Psychophysiology and Biofeedback; 2004. 
132. Escalante A, Lichtenstein MJ, White K, Rios N, Hazuda HP. A method for scoring the pain map of the McGill Pain Questionnaire for use in epidemiologic studies. Aging-Clinical \& Experimental Research 1995;7:358-66.

133. Engle VF, Graney, Fox-Hill, E., \& McKeon, L. Pain quality descriptors for use with Black and White nursing home residents. The Gerontologist 2005;45:75.

134. Herr KA, Spratt K, Mobily PR, Richardson G. Pain intensity assessment in older adults: use of experimental pain to compare psychometric properties and usability of selected pain scales with younger adults. Clinical Journal of Pain 2004;20:207-19.

135. Taylor LJ, Herr K. Pain intensity assessment: a comparison of selected pain intensity scales for use in cognitively intact and cognitively impaired African American older adults. Pain Management Nursing 2003;4:87-95.

136. Melzack R. The short-form McGill Pain Questionnaire. Pain 1987;30:191-7.

137. Idler EL, Benyamini Y. Self-rated health and mortality: a review of twenty-seven community studies. Journal of Health \& Social Behavior 1997;38:21-37.

138. Defining overweight and obesity. Center for Disease Control and Prevention, 2008. (Accessed July 17, 2009 at http://www.cdc.gov/nccdphp/dnpa/obesity/defining.htm.)

139. Garrow JS, Webster J. Quetelet's index (W/H2) as a measure of fatness. International Journal of Obesity 1985;9:147-53.

140. Deathe AB, Miller WC. The L test of functional mobility: measurement properties of a modified version of the timed "up \& go" test designed for people with lower-limb amputations. Physical Therapy 2005;85:626-35.

141. Cohen J. Statistical Power Analysis for the Behavioral Sciences 2nd ed. Hillsdale, NJ: L, Erlbaum Associates; 1992.

142. IRB 005 UTHSC IRB Informed Consent. 2004. (August 29, 2009, at http://www.utmem.edu/research/research_compliance/IRB/docs/sops/SOP05. pdf.)

143. Asano M, Rushton P, Miller WC, Deathe BA. Predictors of quality of life among individuals who have a lower limb amputation. Prosthetics \& Orthotics International 2008;32:231-43.

144. Davies B, Datta D. Mobility outcome following unilateral lower limb amputation. Prosthetics \& Orthotics International 2003;27:186-90.

145. Brooks D, Parsons J, Hunter JP, Devlin M, Walker J. The 2-minute walk test as a measure of functional improvement in persons with lower limb amputation. Archives of Physical Medicine \& Rehabilitation 2001;82:1478-83.

146. De Godoy JMP, Braile DM, Buzatto SHG, Longo O, Jr., Fontes OA. Quality of life after amputation. Psychology, Health \& Medicine 2002;7:397-400.

147. State and County Quick Facts. (Accessed October 14, 2009, at http://quickfacts.census.gov/qfd/.)

148. Green JJ, Kerstetter K, Nylander III AB. Socioeconomic resources and self-rated health: a study in the Mississippi Delta. Sociological Spectrum 2008;28:194-212.

149. Mensah GA, Mokdad AH, Ford ES, Greenlund KJ, Croft JB. State of disparities in cardiovascular health in the United States. Circulation 2005;111:1233-41. 
150. Centers for Disease Control and Prevention. Health disparities experienced by black or African Americans-United States. Morbidity \& Mortality Weekly Report 2005;54:1-3.

151. MacKenzie EJ, Bosse MJ, Kellam JF, et al. Early predictors of long-term work disability after major limb trauma. Journal of Trauma-Injury Infection \& Critical Care 2006;61:688-94.

152. Levine M, Ensom MH. Post hoc power analysis: an idea whose time has passed? Pharmacotherapy 2001;21:405-9.

153. Munce SE, Stewart DE. Gender differences in depression and chronic pain conditions in a national epidemiologic survey. Psychosomatics 2007;48:394-9.

154. Mottram S, Peat G, Thomas E, Wilkie R, Croft P. Patterns of pain and mobility limitation in older people: cross-sectional findings from a population survey of 18,497 adults aged 50 years and over. Quality of Life Research 2008;17:529-39.

155. Finger S, Hustwit MP. Five early accounts of phantom limb in context:Pare,Descartes, Lemos, Bell, and Mitchell. Neurosurgery 2003;52:67586.

156. Treede RD, Kenshalo DR, Gracely RH, Jones AK. The cortical representation of pain. Pain. 1999;79:105-11.

157. Flor H, Elbert, T., Muhlnickel, W., Pantev, C., Wienbruch, C., Taub, E. Cortical reorganization and phantom phenomena in congenital and traumatic upperextremity amputees. Experimental Brain Reseach 1997;119:205-12.

168. Capaday C, Richardson MP, Rothwell JC, Brooks DJ. Long-term changes of GABAergic function in the sensorimotor cortex of amputees. A combined magnetic stimulation and 11C-flumazenil PET study. Experimental Brain Research 2000;133:552-6.

169. Nikolajsen L, Finnerup NB, Kramp S, Vimtrup AS, Keller J, Jensen TS. A randomized study of the effects of gabapentin on postamputation pain. Anesthesiology 2006;105:1008-15. 


\section{APPENDIX A: HISTORY AND ETIOLOGIES OF PHANTOM LIMB PAIN}

Finger and Hustwit compiled a modern history of phantom limb pain. ${ }^{155}$ Ambroise Pare, a physician and surgeon, in publications from 1575described pain being present in the limb after amputation. In 1680, Renee Descartes wrote about phantom pain and the role of nerves and the brain. Aaron Lemos wrote a dissertation in 1798 that described amputees with pain that continued following an amputation. Charles Bell was a physician that wrote about separate functions of the dorsal and ventral root of the spinal cord, and nerve energies. The term phantom limb pain was first used in 1871 by Silas Weir Mitchell, a surgeon during the American Civil War.

This phenomenon of phantom limb pain has been described to occur because of mechanisms in the peripheral and in the central nervous system that sense, signal, and interpret pain. ${ }^{35}$ The somatosensory homunculus is a part of the central nervous system that plays a major role in the phenomenon of phantom limb pain. The somatosensory homunculus is located within the brain. It is organized so that every part of the body corresponds to an area within the somatosensory homunculus.

Although this explanation is simple, the brain's role in interpreting the signal is quite complex. The processing of pain information is a construct of sensorydiscriminative pain and affective-motivational constructs that occur within the primary somatosensory cortex, the secondary somatosensory cortex, parietal operculum, insula, anterior ingulated cortex and prefrontal cortex. ${ }^{156}$ There are areas that are very sensitive to incoming sensations in the body that possess a great amount of nerve endings such as the fingers and lips. When a signal enters the somatosensory homunculus, the pain is felt in the location corresponding to the nerve pathway that delivered the signal.

A nerve that is relatively quite will send a positive signal of pain if it is stimulated. ${ }^{132}$ An ectopic discharge of a nerve occurs when stimulation of the nerve occurs along a nerve tract at a more proximal location than the sensory nerve ending. A positive pain signal is felt at the specific area associated with the sensory nerve ending, although the nerve was stimulated at a more central location of the nerve fiber. This explains part of the mechanism for painful sensation in a phantom limb. If a nerve that would usually have the sensory nerve ending located in a missing limb is stimulated, the pain information is sent to the brain as though it originated in the missing limb.

The phenomena referred to as cross-talk is associated with sensation reception from adjacent receptors. ${ }^{132}$ When information is transmitted through an individual neuron, a synapse, which involves depolarization of the neuron, travels down its axon. The axons of peripheral sensory neurons are insulated with a myelin sheath, but have uninsulated areas where depolarization occurs called the nodes of Ranier. Throughout the body there are nerves that share common areas with other nerve and are very close to each other. A signal traveling through one neuron could stimulate another by depolarizing a neighboring nerve at the nodes of Ranier. This mechanism could also 
amplify a signal if several low threshold nerves stimulated a synapse in a high threshold neuron, so that a relatively benign stimulus might be felt as an intense pain.

Another classification of pain where there is an inconsistency between the location where pain originates and the location where pain is perceived to be from is referred pain. Referred pain is pain that is felt on the skin, although the cause of the pain originates in a visceral organ. ${ }^{132}$ When there is an insult to an organ, there is a painful stimulus that is elicited and is then communicated from the organ to the brain via synapsing nerves. When the pain is felt in a separate area from where the distressed tissue is, this could be caused by a miscommunication that occurs as nerves carry the signal from the site of origin to the somatosensory homunculus in the brain.

Miscommunication can occur between the synapsing nerves within the spinal cord or at the level of the peripheral nervous system. It is also possible that the corresponding sensory areas on the skin are connected through a pathway of the peripheral nerves to the referring organ. A stimulus originating on the organ might stimulate a signal the area on the skin where pain is perceived to originate. Another theory is that there is a connection that occurs between nerve fibers from the two locations, the organ and its corresponding skin sensation area, within the spinal cord. A similar mechanism could play a role in phantom limb pain as sensory nerves in a missing limb have a connection to a corresponding sensory area within the body.

The residual limb has also been shown to contribute to the etiology of phantom limb pain. An increase in surface electromyogram, which measured muscle tension changes in the residual limb of persons with limb loss was associated with cramping phantom limb pain. ${ }^{83}$ A decrease blood flow to the residual limb as measured by thermography is associated with the report of burning phantom limb pain. ${ }^{82}$

Cortical reorganization as evidenced by changes in functional MRI measured by neuromagnetic source imaging has been associated with the presence of phantom limb pain. ${ }^{157}$ Positron emission tomography has shown an up regulation of gammaaminobutyric_acid receptors in the cortex in areas that correspond to missing limbs in persons with limb loss. ${ }^{168}$ This provides neuorchemical evidence for cortical reorganization after limb loss. Drugs that mimic the effect of gamma-aminobutyric acid are commonly prescribed to treat and prevent phantom limb pain, although there is evidence this may not be effective. ${ }^{159}$

The above writings present possible mechanisms to explain phantom limb pain. The nervous system is complex and dynamic. Pain is a construct that has behavioral and physical components. The current lack of understanding for how phantom limb pain occurs partially because is that is extremely difficult to account for all of the factors that influence pain at a given time. The somatosensory homunculus functions within several structures of the brain, and has a complex function in interpreting the emotional component of pain as well as pain location. The peripheral nervous system may contribute to pain through the mechanisms discussed above or through unknown mechanisms. Additionally, phantom limb pain may have several etiologies, and an 
individual with limb loss may have more than one of the etiologies that contribute to their phantom limb pain. 


\section{APPENDIX B: UNIVERSITY OF TENNESSEE HEALTH SCIENCE CENTER INSTITUTIONAL REVIEW BOARD APPROVAL}

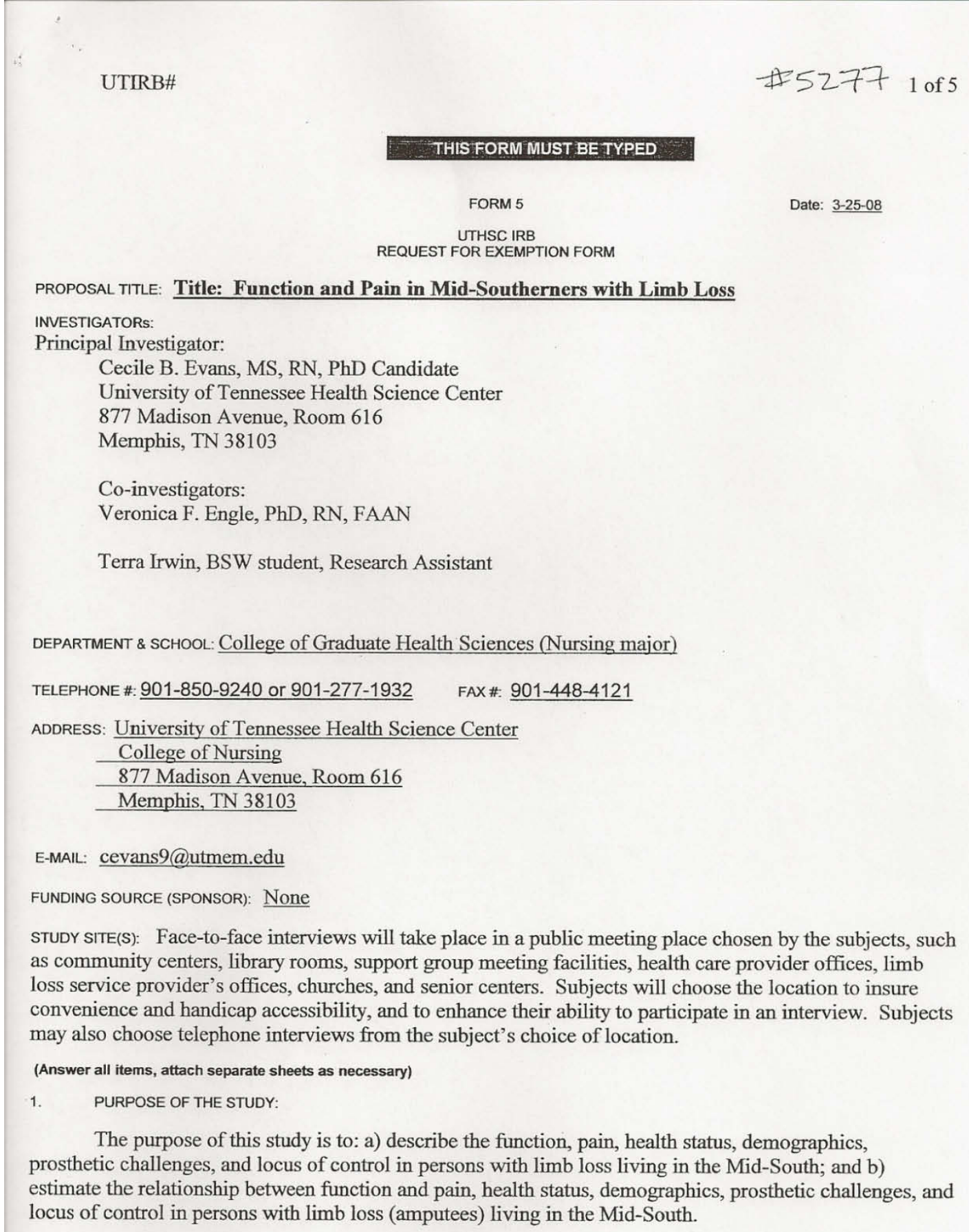
as community centers, library rooms, support group meeting facilities, health care provider offices, limb loss service provider's offices, churches, and senior centers. Subjects will choose the location to insure convenience and handicap accessibility, and to enhance their ability to participate in an interview. Subjects may also choose telephone interviews from the subject's choice of location.

(Answer all items, attach separate sheets as necessary)

1

PURPOSE OF THE STUDY:

The purpose of this study is to: a) describe the function, pain, health status, demographics, prosthetic challenges, and locus of control in persons with limb loss living in the Mid-South; and b) estimate the relationship between function and pain, health status, demographics, prosthetic challenges, and locus of control in persons with limb loss (amputees) living in the Mid-South.

Prepared: $3-25-8$ 
2. DESCRIPTION OF SUBJECT POPULATION AND METHOD(S) OF SUBJECT RECRUITMENT:

(Include an explanation how recruitment of subjects is intended to satisfy NIH or FDA requirements for the inclusion of women, racial/ethnic groups and children in human subjects research).

This study will include 100 Mid-Southerners with limb loss. Subjects need to be at least 18 years of age, as the measurement of function for children is different than the measurement function for adults. Both genders are included and all races. Subjects must have limb loss at the level of at least a hand or foot. Limb loss can be for any reason. Subjects do not have to be in pain to be in the study. All subjects will be community dwelling at the time of the study, and must be at least six months from the date of the last amputation or revision.

\section{Inclusion criteria include:}

1. Community-dwelling

2. Persons with upper, lower, or both upper and lower limb loss

3. Limb loss at a higher level than a finger or toe

4. Limb loss for any reason

5. Reside in the Mid-South, including the states of Alabama, Arkansas, Kentucky, Louisiana, Mississippi, Missouri, and Tennessee

6. Either gender

7. All races

8. Age 18 years or older

9. Be at least six months from the last limb amputation or revision

10. Understand spoken English

11. Able to communicate verbally

12. Provide informed consent

A purposive, convenience, snowball sampling technique will be used to recruit subjects. Persons with limb loss will be recruited through contacting key persons from the following types of organizations: limb loss support groups, national limb loss advocacy groups, and limb loss service providers. Recruitment strategies for each type of organization will be discussed below.

Limb loss support group coordinators will contact support group members by phone, email, or traditional mail with information about the study. This will keep the member's personal information confidential from the principal investigator as she will be contacted only by persons who express interest in the study.

Limb loss service providers will be identified through a snow ball technique, and given information about the study. Limb loss service providers include prosthetists, diabetic supply services, occupational or physical therapists, primary or specialty health care providers, social support services, advocacy groups, medical suppliers, and sports organizations. Key persons within these organizations will be contacted in person, by telephone, fax, or email. Limb loss service providers may provide information about the study to subjects who will then contact the principal investigator directly. Providers who are willing may place posters and/or brochures in a prominent place where they can be viewed by their clients in a public area. Persons with limb loss interested in participating in the study will contact the principal investigator directly by phone or email.

Inclusion of Women: Limb loss occurs more frequently in men than women. However, women have been under represented in previous studies. Recruiting will be aimed at both men and women.

Prepared 3-25-08 
Inclusion of Minorities: All ethnicities/races will be included. African Americans with diabetes have about twice the risk of having an amputation than Whites with diabetes, and may be under represented in studies. Recruiting materials include a posters that is specifically aimed at recruiting African American amputees.

Inclusion of Children: Children under the age of 18 have different roles and therefore different standards of which to estimate function. Children under the age of 18 will be excluded from this study.

Data collection procedure involves a one hour, one time interview face-to-face or via telephone. Subjects contact the principal investigator directly and will be screened using the above inclusion criteria. Subjects who meet the inclusion criteria will make an appointment for a face-toface interview in a public place of their choice or a telephone interview.

Face-to-face interview will take place in a public meeting place chosen by the subjects, such as community centers, library rooms, support group meeting facilities, health care provider offices, limb loss service provider's offices, churches, and senior centers. Subjects will choose the location to insure convenience, handicap accessibility, enhance their ability to participate in an interview. Subjects may also choose telephone interviews from the subject's choice of location.

A master list of study subjects will be maintained during the study to avoid duplicating surveys. This will be maintained in a locked file cabinet and will be destroyed after the study is completed. Individual surveys will be assigned an identifier number and there will be no information that could connect subjects with their responses.

Face to face interview procedure:

1. Meet the subject at the agreed upon time and place.

2. Introduce self to subjects.

3. Provide explicit explanation of the research study and obtain verbal consent.

4. Conduct structured interview that includes demographic information from subjects, the SIP68 , select questions from the SIP-68 specific to limb loss, pain map drawing of pain location, pain quality assessment, pain intensity assessment, pain pattern assessment, prosthetic challenges, God Locus of Health Control, and foot care questions.

5. Give the subject a thank you letter for their participation.

6. All data will be kept in a locked file cabinet.

7. Subjects will be mailed a summary of the study results.

8. List of subjects will be destroyed.

The procedure for the telephone interview was as follows:

1. Contact subject at an agreed upon time.

2. Introduce self to subjects

3. Provide explicit explanation of the research study and obtain verbal consent.

4. Conduct structured interview that includes demographic information from subjects, the SIP-68, Select questions from the SIP-68 specific to limb loss, pain location, pain quality assessment, pain intensity assessment, pain pattern assessment, prosthetic challenges, God Locus of Health Control, and foot care questions.

5. Send the participants a thank you letter for their participation.

Prepared 3-25-08 
6. All data will be kept in a locked file cabinet.

7. Subjects will be mailed a summary of the study results.

8. List of subjects will be destroyed.

Verbal informed consent, verbal survey, and copy of McGill pain map is attached.

The research involves only the use of verbal survey procedures. The human subjects can't be identified directly or indirectly through identifiers. Individual subjects will not be identified in any presentation or publications based on the results of this study. HIPAA authorization is not needed as this study requires no medical record information, and no documentation that subjects were in a study will be added to their medical record.

A list of subject names will be available only to research staff during the study to prevent duplicating surveys. The master list will be kept in a locked file and destroyed after completion of the research study.

c. The study qualifies for exempt status under 45CFR46.101(b). (Indicate category $(b)(1)$ or $(b)(2)$, etc.)

This research study qualifies for exempt status under category (b)(2) as in involves the use of interview procedures, and information is recorded in a way that human subjects can't be identified, and the responses of the research outside the research could reasonably place the subjects at risk of criminal or civil liability or be damaging to the subjects' financial standing, employability, or reputation.

6. INFORMED CONSENT: Informed consent is generally required for an exempt study unless it cannot practicably be carried out without a waiver of consent. Please check the appropriate box.

b. The study can be undertaken even if informed consent is required.

$\underline{\text { Yes }}$

Verbal consent for subject participation conducted face-to-face and by telephone will be obtained prior to beginning survey, and will be evident by participation in the survey interview.

Prepared 3-25-08 


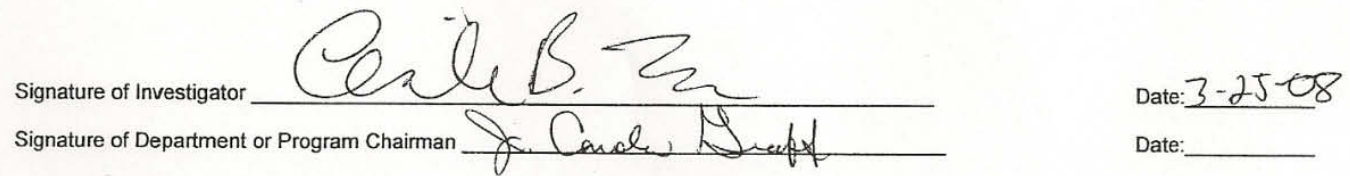

Typewritten Name of Program Chairman: $\underline{\text { J. Carolyn Graff, } \mathrm{PhD}, \mathrm{RN}, \mathrm{FAAIDD}}$

(The IRB reserves the right to request the investigator to provide additional information concerning the proposal.)

Submission requires original and 2 copies.

DO NOT WRITE BELOW THIS LINE

\begin{tabular}{lll}
\hline & DO NOT WRITE BELOW THIS LINE & \\
IRB ACTION: & Approved & Approved w/proviso(s)
\end{tabular}

Consent Required:

No __ Yes __ Not Applicable

Written _ Signed _ _ _

IRB Reviewer:

Title

Date

(Rev. 11/2003)

Prepared 3-25-08 
Principal Investigator:
Brief Project Title: $\quad$ Function and Pain in the Mid-Southerners with Limb Loss

IRB ACTION: Approved_________ Approved w/proviso(s) _____ Referred For Board Review ____ _

COMMENTS: This study qualifies for exempt status under 45CFR46.101(b)(2). In accord with

45CFR46.116(d), informed consent may be altered with the consent cover statement used in lieu of a

consent interview. The requirement to secure a signed consent form is waived under 45CFR46.117(c)(2).

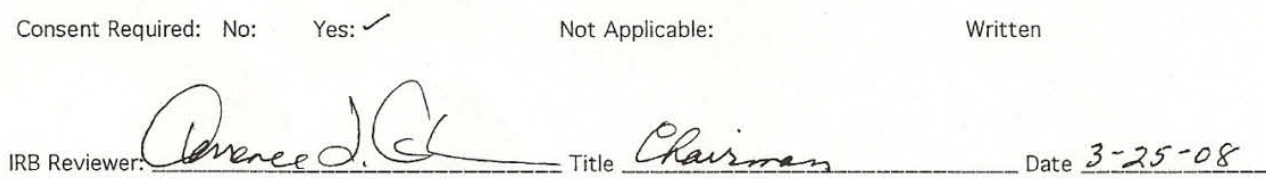


This interview is part of a research study. The purpose of this study is to describe everyday activities, pain, and health in persons with limb loss. It is being conducted by Cecile Evans, a nurse practitioner, as part of her doctoral studies at the University of Tennessee Health Science Center. You will answer questions about your everyday activities, your limb loss, your health, and your pain. I will talk with you one time for about an hour. There are few risks for being in this study. You may become tired from answering the questions. You may get upset discussing your pain. If you get tired or upset, you can stop the interview at any time. Being in this study may help you talk about your pain with your doctor. Being in this study is voluntary. Not being in the study won't affect your care. Nothing will connect your name to your answers. A list of participants will be kept in a locked file cabinet to insure your privacy. This list will be destroyed when the study is complete. 


\section{APPENDIX D: STUDY INSTRUMENT}

\section{Demographics}

1. Filled out by interviewer, Today's date:

2. Filled out by interviewer, Study number:

3. Filled out by interviewer: Telephone method: $\mathbf{0}=$ Face to face, $\mathbf{1}=$ By phone

4. What is your age in years (how old are you)?

5. Gender: $\mathbf{0}=$ Female, $\mathbf{1}=$ Male

6. How do you describe your ethnicity or race?

$\mathbf{1}=$ White, $\mathbf{2}=$ Black, $\mathbf{3}=$ Latino, $\mathbf{4}=$ Asian,

$\mathbf{5}=$ Native American, $\mathbf{6}=$ other or mixed (Specify):

7. How many years of school did you complete? 


\section{Health Status}

1. How would you rate you health?

$$
\begin{aligned}
& \mathbf{0}=\operatorname{Bad}(\text { Bad health only) } \\
& \mathbf{1}=\text { Poor (Small amount of health) } \\
& \mathbf{2}=\text { Fair (Medium amount of health) } \\
& \mathbf{3}=\text { Good (Large amount of health) } \\
& \mathbf{4}=\text { Excellent }(\text { As good as health can be) }
\end{aligned}
$$

2. Do you have sugar diabetes? $\mathbf{0}=\mathbf{N o}, \mathbf{1}=$ Yes

3. Do you smoke now? $\quad \mathbf{0}=$ No, $\mathbf{1}=$ Yes

4. Have you ever smoked? $\quad \mathbf{0}=\mathbf{N o}, \mathbf{1}=$ Yes

5. How tall are you?

6. How much do you weigh? 


\section{SIP68 Somatic Autonomy}

SA1. I get around in a wheelchair.

SA2. I get dressed only with someone's help.

SA3. I do not move into or out of bed by myself, but am moved by a person or mechanical aid.

SA4. I stand up only with someone's help.

SA5. I do not fasten my clothing, for example, require assistance with buttons, zippers, shoelaces.

(I need help with buttons, zippers, shoelaces, I can't make my clothes come together.)

SA6. I do not walk at all.

SA7. I do not use stairs at all. (I do not use steps at all.)

SA8. I make difficult moves with help, for example, getting into or out of cars, bathtubs.

SA9. I do not bathe myself completely, for example, require assistance with bathing. (I do not take a bath all by myself, I need some help when I take a bath.)

SA10. I do not bathe myself at all, but am bathed by someone else. (I don't take a bath all by myself, but am bathed by someone else.)

SA11. I do not have control of my bladder.

(I do not have control of my pee, I wet myself.)

SA12. I do not have control of my bowels.

(I do not have control of my poop, I poop in my pants?)

SA13. I am very clumsy in body movements.

SA14. I feed myself with help from someone else.

SA15. I do not maintain balance.

(I do not keep my balance, I can't keep my balance.)

(SA continued next page) 
SA16. I use bedpan with assistance.

(I use a bedpan with help)

SA17. I change position frequently.

(I turn from side to side a lot.) 


\section{SIP68 Mobility Control}

MC1. I go up and down stairs more slowly, for example, one step at a time, stop often.

MC2. I walk shorter distances or stop to rest often.

MC3. I walk more slowly.

MC4. I use stairs only with mechanical support, for example, handrail, cane, crutches.

MC5. I walk by myself but with some difficulty, for example, limp, wobble, Stumble, have stiff leg.

(I walk by myself but with some trouble, for example, with a wobble, stumble or a stiff leg.)

MC6. I kneel, stoop, or bend down only by holding on to something. (I swat only by holding on to something)

MC7. I do not walk up or down hills.

MC8. I get in or out of chairs by grasping something for support, or using a cane or walker.

(I get in or out of chairs by holding onto something or by using a cane or walker.)

MC9. I stand only for short periods of time.

MC10. I dress myself, but do so very slowly.

MC11. I have difficulty doing handwork, for example turning faucets, using kitchen gadgets, sewing, carpentry.

MC12. I move my hands or fingers with some limitation or difficulty. (I move my hands or fingers to a certain point without problems.) 


\section{SIP68 Mobility Range}

MR1. I am not doing any of the shopping that I would usually do.

MR2. I am not going into town.

MR3. I am not doing any of the house cleaning that I would usually do.

MR4. I am not doing any of the regular work around the house that I would usually do.

(I am not doing what I would always do around the house.)

MR5. I stay home most of the time.

MR6. I am not doing any of the clothes washing that I would usually do. (I don't keep my clothes clean.)

MR7. I am not going out to visit people at all.

MR8. I am getting around only within one building. (I am getting around only within one house/apartment.)

MR9. I have given up taking care of personal or household business affairs, for example, paying bills, banking, working on a budget.

MR10. I do not get around in the dark or in unlit places without someone's help.

(I do not get around without help in the dark.) 


\section{SIP68 Psychic Autonomy and Communication}

PAC1. I have difficulty reasoning and solving problems, for example, making plans, making decisions, learning new things.

(I have problems with trying to understand new things.)

PAC2. I have difficulty doing activities involving concentration and thinking.

(I have problems doing things that require thinking.)

PAC3. I react slowly to things that are said or done.

(I am slow to things that are said and done, I am slow about catching on.)

PAC4. I make more mistakes than usual.

PAC5. I do not keep my attention on any activity for long.

(I do not keep my mind on an activity for long. I do not keep

my interest on an activity for long.)

PAC6. I forget a lot, for example, things that happened recently, where I put things, appointments.

PAC7. I am confused and start several actions at a time. (I do try to do too many things at one time.)

PAC8. I do not speak clearly under stress.

(When I am upset, it is hard to understand what I say.)

PAC9. I have difficulty speaking for example, get stuck, stutter, stammer, slur my words.

(I have problems talking, for example, get stuck, stutter, stammer, slur my words.)

PAC10. I do not finish things I start.

PAC11. I am having trouble writing or typing. 


\section{SIP68 Social Behavior}

SB1. My sexual activity is decreased.

(My sex life is not doing too good.)

SB2. I am cutting down the length of visits with friends.

(I don't go visit as much as I used to with friends.)

SB3. I am drinking less fluids.

(I am drinking less water, tea, coffee, that kind of thing.)

SB4. I am doing fewer community activities.

(I am doing less with church, school, or volunteer work.)

SB5. I am doing fewer social activities with groups of people.

(I am doing less with family and friends.

SB6. I am going out for entertainment less often.

(I am going out less. I am going out less to kick it.)

SB7. I stay away from home only for brief periods of time.

(I don't stay away from home long.)

SB8. I am eating much less than usual.

(I don't eat as much as I was doing.)

SB9. I am not doing heavy work around the house.

SB10. I do my hobbies and recreation for shorter periods of time. (I do fun things for shorter times.)

SB11. I am doing less of the regular daily work around the house than I would usually do.

SB12. I am cutting down on some of my usual inactive recreation and pastime, for example, watching TV, playing cards, reading. (I do less that is not physically active like watching TV, playing cards, reading.) 


\section{SIP68 Emotional Stability}

ES1. I often act irritable toward those around me, for example, snap at people, give sharp answers, criticize easily.

(I am not nice or rude to others a lot.)

ES2. I act disagreeable to family members, for example, I act spiteful, I am stubborn.

(I don't agree with family members, I am mean, selfish, a bitch.)

ES3. I have frequent outbursts of anger at family members, for example, strike at them, scream, throw things at them.

(I am angry at family members a lot, for example, swing at them, or try to hit them.)

ES4. I act irritable and impatient with myself, for example, talk badly about myself, swear at myself, blame myself for things that happen. (I am a bitch with myself.)

ES5. I am not joking with family members as I usually do. (I am not goofing/kidding/playing around with family members as much.)

ES6. I talk less with those around me. (I rap less with those around me.) 


\section{Limb Loss Impact Profile 12}

1. I make difficult moves with help, for example, getting into or out of cars, bathtubs since I lost my arm or leg. (SA8)

2. I do not maintain balance since I lost my arm or leg. (SA15)

3. I walk more slowly since I lost my arm or leg. (MC3)

4. I dress myself, but do so very slowly since I lost my arm or leg. (MC10)

5. I am not doing any of the regular work around the house that I would usually do since I lost my arm or leg. (MR4) (I am not doing what I would always do around the house because I lost my leg/arm.)

6. I stay home most of the time since I lost my arm or leg. (MR5)

7. I have difficulty doing activities involving concentration and thinking since I lost my arm or leg. (PAC2)

8. I forget a lot, for example, things that happened recently, where I put things, appointments since I lost my arm or leg. (PAC6)

9. I am doing fewer social activities with groups of people since I lost my arm or leg. (SB5)

(I am going out less. I am going out less to kick it.)

10. I am doing less of the regular daily work around the house than I would usually do since I lost my arm or leg. (SB11)

11. I often act irritable toward those around me, for example, snap at people, give sharp answers, criticize easily because I lost my arm or leg. (ES1)

(I am not nice or rude to others a lot because I lost my leg/arm.)

12. I act irritable and impatient with myself, for example, talk badly about myself, swear at myself, blame myself for things that happen since I lost my arm or leg. (ES4) (I am a bitch with myself.) 


\section{Health Status Amputation History}

1. How many limbs have you lost?

2. At what area on your arm/leg was your limb lost?

1. At right hand 2. Below right elbow 3. At right elbow

4. Above right elbow 5. Right Shoulder

6. At right foot 7. Below right knee 8. At right knee

9. Above right knee 10. Pelvis- right side

11. At left hand 12. Below left elbow 13. At left elbow

14. Above left elbow 15. Left Shoulder

16. At right foot 17. Below right knee 18. At right knee

19. Above right knee 20. Pelvis- right side

3. Why did you lose your limb ( $\mathrm{arm} / \mathrm{leg})$ ? (Record exact words)

4. When did you lose this limb ( $\mathrm{arm} / \mathrm{leg})$ ?

5. Do you feel pain in your lost limb(s) ( $\mathrm{arm} / \mathrm{leg})$ ?

$$
\mathbf{0}=\mathbf{N o}, 1=\text { Yes }
$$




\section{Phantom Limb Pain}

PLP1. Rate you pain in this missing limb right now:

$\mathbf{0}=$ No pain, $\mathbf{1}=$ Small, $\mathbf{2}=$ Medium,

$\mathbf{3}=$ Large, $\mathbf{4}=$ As bad as it can be

PLP2. How much pain do you usually have in these areas?

$\mathbf{0}=$ No pain, $\mathbf{1}=$ Small, $\mathbf{2}=$ Medium,

$\mathbf{3}=$ Large, $\mathbf{4}=$ As bad as it can be

PLP3. What is the worst (largest) pain you have in this missing limb?

$\mathbf{0}=$ No pain, $\mathbf{1}=$ Small, $\mathbf{2}=$ Medium,

$\mathbf{3}=$ Large, $\mathbf{4}=$ As bad as it can be

PLP4. What is the least amount (smallest) pain you have in this missing limb:

$\mathbf{0}=$ No pain, $\mathbf{1}=$ Small, $\mathbf{2}=$ Medium,

$\mathbf{3}=$ Large, $\mathbf{4}=$ As bad as it can be

PLP5. Does the pain in this missing limb come and go?

$\mathbf{0}=\mathbf{N o}, 1=$ Yes

PLP6. Is there pain in this missing limb there all the time?

$\mathbf{0}=\mathbf{N o}, \mathbf{1}=$ Yes

Do you ever have any of these feelings in your missing limb?

PLP7. Burning

PLP9. Stabbing

PLP11. Tiring

PLP13. Abnormal Position
PLP8. Cramping

PLP10. Shocking/Shooting

PLP12. Twisting

PLP14. Tight Band Around Area 


\section{Residual Limb Pain}

RLP1. Do you have pain in the remaining portion of your lost limb(s)?

$\mathbf{0}=\mathbf{N o}, \mathbf{1}=$ Yes

If no, skip to other pain assessment.

RLP2. If more than one limb lost:

Which limb has the most residual limb (stump) pain?

$1=$ Right arm, $2=$ Left arm, $3=$ Right leg, $4=$ Left leg

The following questions will be about this limb (arm/leg):

RLP3. Rate you pain in this residual limb right now:

$\mathbf{0}=$ No pain, $\mathbf{1}=$ Small, $\mathbf{2}=$ Medium,

$\mathbf{3}=$ Large, $\mathbf{4}=$ As bad as it can be

RLP4. How much pain do you usually have in this residual limb?

$\mathbf{0}=$ No pain, $\mathbf{1}=$ Small, $\mathbf{2}=$ Medium,

$\mathbf{3}=$ Large, $\mathbf{4}=$ As bad as it can be

RLP5. What is the worst (largest) pain you have in this residual limb?

$\mathbf{0}=$ No pain, $\mathbf{1}=$ Small, $\mathbf{2}=$ Medium,

$\mathbf{3}=$ Large, $\mathbf{4}=$ As bad as it can be

RLP6. What is the least amount (smallest) pain you have in this

residual limb?

$\mathbf{0}=$ No pain, $\mathbf{1}=$ Small, $\mathbf{2}=$ Medium,

$\mathbf{3}=$ Large, $\mathbf{4}=$ As bad as it can be

RLP7. Does the pain in this residual limb come and go?

$\mathbf{0}=$ No, $1=$ Yes

RLP8. Is there pain in this residual limb there the time?

$\mathbf{0}=\mathbf{N o}, 1=$ Yes

Do you ever have any of these feelings in your residual limb?

RLP9. Burning

RLP11. Stabbing

RLP13. Tiring

RLP15. Abnormal Position
RLP10. Cramping

RLP12. Shocking/Shooting

RLP14. Twisting

RLP16. Tight Band Around Area 


\section{Other Pain}

OP1. Do you have pain in other parts of your body?

$\mathbf{0}=\mathbf{N o}, 1=$ Yes

If no, skip other pain assessment.

OP2. Where is your worst (largest) other pain?

The following questions will be about this painful area:

OP3. Rate your pain here right now:

$\mathbf{0}=$ No pain, $\mathbf{1}=$ Small, $\mathbf{2}=$ Medium,

$\mathbf{3}=$ Large, $\mathbf{4}=$ As bad as it can be

OP4. How much pain do you usually have here?

$\mathbf{0}=$ No pain, $\mathbf{1}=$ Small, $\mathbf{2}=$ Medium,

$\mathbf{3}=$ Large, $\mathbf{4}=$ As bad as it can be

OP5. What is the worst (largest) pain you have here?

$\mathbf{0}=$ No pain, $\mathbf{1}=$ Small, $\mathbf{2}=$ Medium,

$\mathbf{3}=$ Large, $\mathbf{4}=$ As bad as it can be

OP6. What is the least amount (smallest) pain you have here?

$\mathbf{0}=$ No pain, $\mathbf{1}=$ Small, $\mathbf{2}=$ Medium,

$\mathbf{3}=$ Large, $\mathbf{4}=$ As bad as it can be

OP7. Does the pain here come and go?

$\mathbf{0}=$ No, $1=$ Yes

OP8. Is there pain there all the time?

$\mathbf{0}=$ No, $1=$ Yes

Do you ever have any of these feelings in this area?

OP9. Burning

011. Stabbing

OP13. Tiring

OP15. Abnormal Position
OP10. Cramping

OP12. Shocking/Shooting

OP14. Twisting

OP16. Tight Band Around Area 
MCGILL PAIN QUESTIONNAIRE PAIN MAP

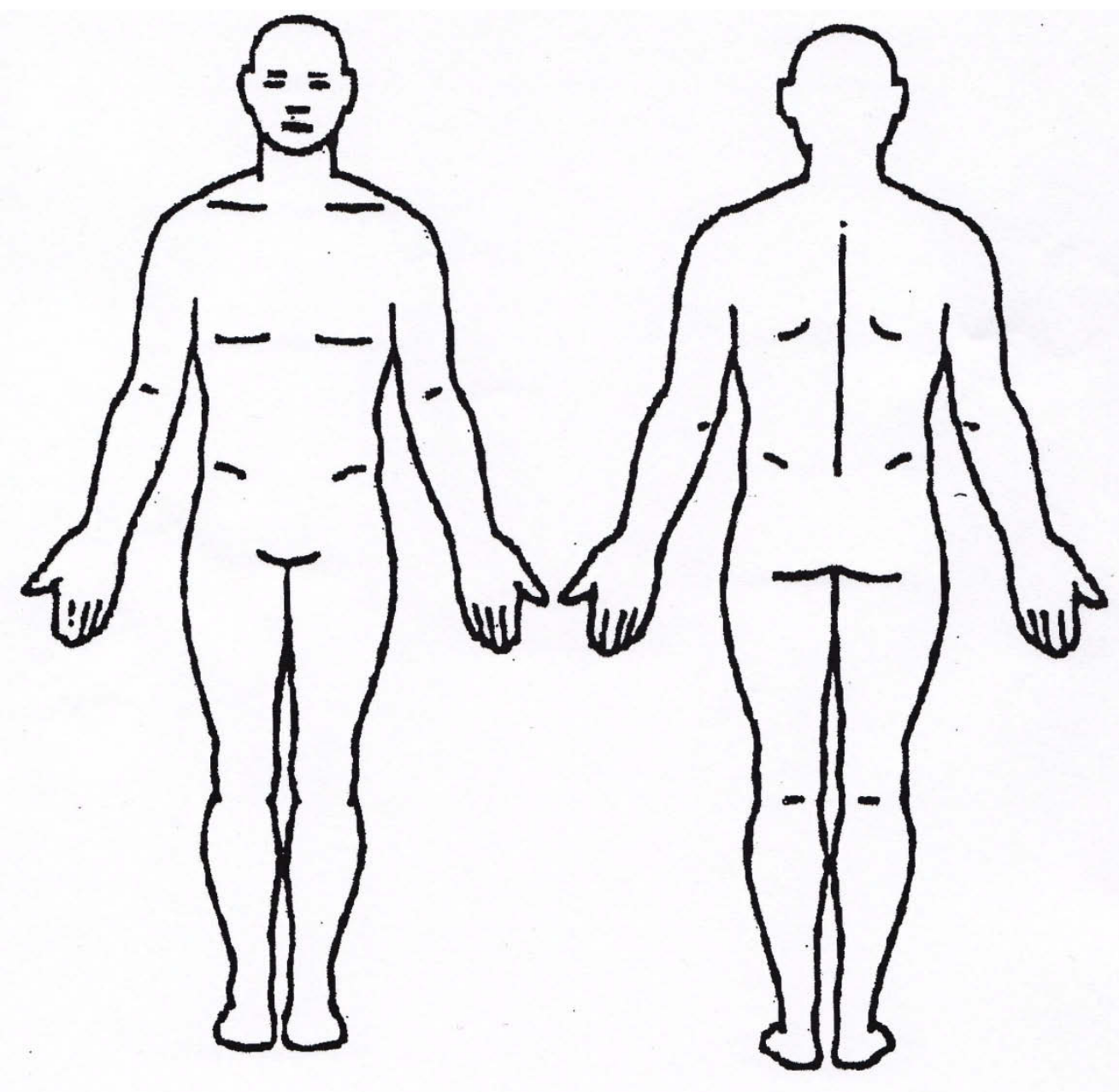


MCGILL PAIN QUESTIONNAIRE PAIN MAP ESCALANTE GRID FOR ANALYSES

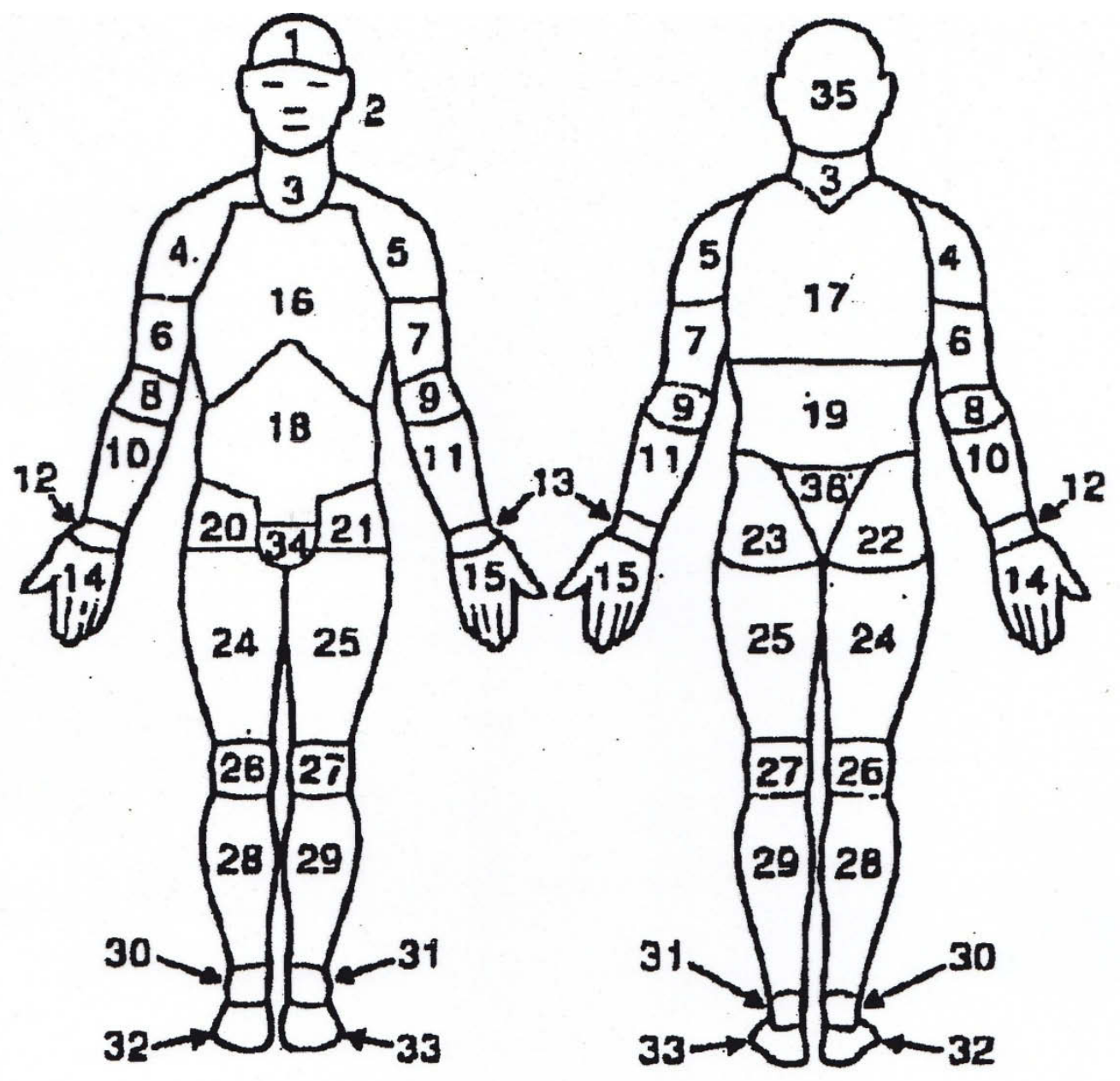




\section{APPENDIX E: DETAILED RECRUITING AND RECRUITMENT MATERIALS}

The American Amputee Foundation (AAF) is a national limb loss advocacy foundation that provides information, referral, peer counseling, self-help literature, and education to persons with limb loss and their families. The AAF assisted with recruiting by offering information to persons attending support groups and though personal mailings to persons with limb loss in the Mid-South. The AAF staff was provided with University of Tennessee Health Science Center (UTHSC) IRB approved letters to persons with limb loss, and appropriate mailing supplies by the PI. The AAF sent out two mailings, to persons with limb loss within the Mid-South. The mailers were sent in late May of 2008, and September 2008.

The amputee support group Out on a Limb (OOAL) also assisted with recruiting. Out on a Limb is a limb loss support group located in Memphis, which is sponsored by Jewish Family Services of Memphis. The PI was able to recruit at monthly meetings by verbally promoting the study and handing out UTHSC IRB approved brochures. Out on a Limb also emailed the UTHSC IRB approved letter to limb loss persons and poster to their email list. The email list includes persons with limb loss, families of persons with limb loss, and professionals who work with persons with limb loss in the greater Memphis area, as well as rural areas surrounding Memphis.

The HealthSouth support group in Memphis also assisted with recruiting limb loss participants. HealthSouth provides both inpatient and outpatient rehabilitation to the general population. The HealthSouth sponsors the support group for all persons with limb loss to attend, both inpatient clients and community dwelling persons with limb loss that may or may not be receiving services from HealthSouth. Most attendees are from the Central Memphis area. UTHSC IRB approved brochures were displayed at the reception desk of the outpatient gym. The support group facilitator offered the UTHSC IRB approved recruitment brochures, at monthly support group meetings.

The Christian Amputee Support Team (CAST) is a national ministry that provides support to persons with limb loss consistent with Christian beliefs and spirituality. Members of CAST in the Mid-south who had email were sent a UTHSC IRB recruiting poster. Persons with limb loss who live in the Mid-South that did not have email were contacted by the coordinator of CAST and given information about the study.

Upper-Ex National Outreach Coalition is a national organization dedicated to serving persons with upper extremity limb loss through support and advocacy. UpperEx placed a full-page copy of the UTHSC IRB recruiting poster in their web-based ejournal for the Sept/Oct and Nov/Dec issues.

The Amputee Coalition of America (ACA) is a national, non-profit amputee consumer educational organization representing people who have experienced amputation or are born with limb differences. The ACA placed an article about the 
study in the news flash section of their website. This included information about the study and how to contact the PI. The marketing department at the University of Tennessee Health Science Center in Memphis released information regarding the study with a UTHSC IRB approved public service announcement. This was released to the University of Tennessee health care partners including the local Memphis Veterans Administration, the Regional Medical Center at Memphis, Methodist Healthcare, and the UT Medical Group. The public service announcement also was released to local media outlets including the city schools radio station, the Memphis Silver Star News, and the Tri-State Defender. The Memphis Silver Star News and the Tri-State Defender are local Black newspapers.

All of the prosthetic limb practices that were listed in the Memphis Yellow pages were contacted, and asked to display recruitment brochures and or refer study participants. Prosthetic limb practices that participated in recruiting persons with limb loss included: Advance Prosthetic and Orthotic; Biodesigns Prosthetics; CFI Prosthetics; Hanger Prosthetics and Orthotics; Precision Prosthetics; Snells Prosthetics and Orthotics; Spears Prosthetics and Orthotics and Wolfchase Limb and Brace. One practice declined to participate, and one practice was not reached by phone. Snells Prosthetics and Orthotics in North Little Rock also displayed UTHSC IRB approved recruitment brochures.

The Diabetes Store displayed UTHSC IRB approved recruitment brochures at their diabetes teaching classroom. They also included the brochure in pharmacy mailings for 300 orders.

Capabilities for Living is an occupational therapy practice that specializes in restoring function to persons with traumatic limb loss. Capabilities for Living displayed the UTHSC IRB approved recruiting brochures. The PI was allowed to interview willing participants on site in a quiet location to insure privacy.

Feather CaseManagement and Consulting sent the UTHSC IRB approved letter to health care provider and recruiting poster to the case managers and providers on their email list. This included nurse case managers as well as other health care providers.

Dr. Veronica Engle sent an email out to the nursing list serve at the UTHSC College of Nursing. This allowed nursing staff and students to recruit through their practice sites.

The TK Martin Center for Technology and Disability at Mississippi State is a direct clinical service center which brings the benefits of assistive technology and an interdisciplinary team to persons with disabilities. They were given UTHSC IRB approved recruitment brochures to distribute, and were emailed the letter to health care providers and recruitment poster.

Twenty-one local limb loss support groups that published information on the UpperEx or AAF websites and were located within the Mid-south were attempted to be 
contacted by phone. Nine had telephone numbers that were disconnected or had cancelled the support group, and five were left messages that they did not return. Four of these contacts welcomed more information by email, and two requested that information be sent by postal mail.

\section{University of Tennessee Health Science Center Institutional Review Board approved recruitment material follows:}


University of Tennessee Health Science Center

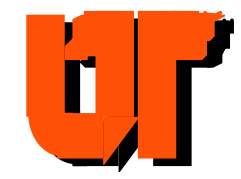

(Today's Date)

Hello,

I am a family nurse practitioner and $\mathrm{PhD}$ student at the University of Tennessee Health Science Center. I am inviting you to be in my $\mathrm{PhD}$ dissertation research study on amputees' pain and everyday activities. I am interested in your pain because pain after a limb loss can make life hard.

If you are over 18 years old, I would like to talk with you about your pain and every day activities, and learn your personal story. This will take about 1 hour and can be done at a place that is convenient for you or using the telephone. You do not have to be in pain to be in this study!

If you are interested in being in this study of have questions about the study, please call me at 901-647-8278. I look forward to talking with you soon about your pain and everyday activities.

Sincerely,

Cecile Evans, RN, FNP, APRN,BC, PhD Student University of Tennessee Health Science Center

877 Madison Avenue, Room 616

Memphis, TN 38103

cevans9@utmem.edu

901-647-8278 


\section{University of Tennessee Health Science Center}

\section{Limb Loss Study}

Do I qualify to be in this study?

o If you are an amputee and are over 18 years of age, and live in Alabama,

Arkansas, Kentucky, Louisiana, Mississippi, Missouri, or Tennessee.

o You do not have to be in pain to participate.

\section{What do I have to do to be in this study?}

○ Answer questions by phone or in person. This takes about an hour.

Who is conducting the study?

- Cecile Evans, Family Nurse Practitioner.

- PhD student at University of Tennessee Health Science Center.

- Professional advisor to Out on a Limb, a limb loss support group.

- Often speaks with limb loss support groups on phantom limb pain.

- Has worked with persons with limb loss for over 15 years.

- Why is this study being done?

- The purpose of this study is to learn more about pain and everyday activities in persons with limb loss.

- This may help doctors and nurses treat persons with limb loss better.

- This study is part of Cecile's $\mathrm{PhD}$ program.

How do I enter this study?

Please call:

Cecile Evans, RN, FNP-BC, PhD student, 901-647-8278

877 Madison Avenue, Room 616, Memphis, TN 38103

cevans9@utmem.edu
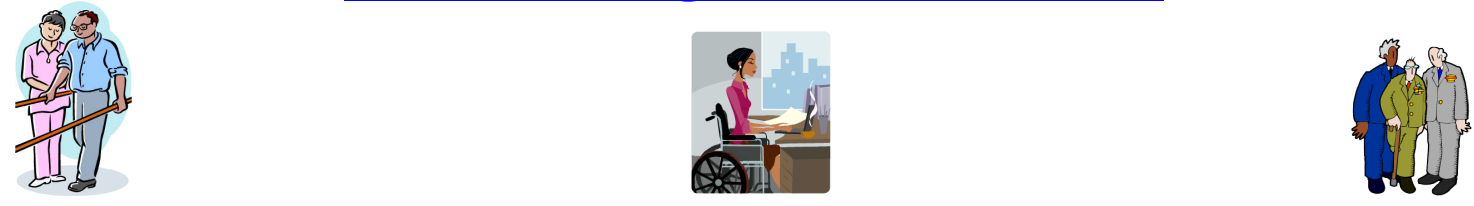


\section{VITA}

Cecile B. Evans was born in Washington D.C. in 1960. She earned a BA in Psychology at the University of Colorado, Boulder, in 1985. Her undergraduate honors thesis was Oxotremorine effects and muscarinic receptor subtypes. At the University of Colorado Health Sciences Center, Denver, School of Nursing, Ms. Evans earned a BSN in 1996, and a MS, Family Nurse Practitioner, in 2000. Her professional nursing practice has included primary care, long-term care, rehabilitation, oncology, and hospice, working with both English- and Spanish-speaking clients. She is an ANCC certified family nurse practitioner.

Ms. Evans's professional research experience includes four years at the Institute for Behavioral Genetics at the University of Colorado, where she worked on research projects that studied cholinergic and GABA neurotransmitters. Ms. Evans's experience includes assisting in a clinical pain laboratory that studied phantom limb pain, headaches, low back pain, and RSD in a military tertiary care medical center. As a graduate student, she worked as a research assistant organizing and cataloging qualitative photographic data for a research project that studied transitions in older rural Hispanics. She has experience as a research nurse practitioner and IRB member at National Jewish Medical and Research Center. Ms. Evans has co-authored publications on cholinergic receptor sub-types, the psychosphysiology of phantom limb pain, and RSD. Her recent presentations include the topics of pain in African Americans, community reintegration of persons with limb loss, and function and pain in MidSoutherners with limb loss. Ms. Evans is frequently asked to speak at limb loss support groups on phantom limb pain.

Ms. Evans is a member of the American Diabetes Association, American Nursing Association, American Society for Pain Management Nursing, Amputee Coalition of America, Gerontological Society of America, and Sigma Theta Tau. She serves on the research committee at American Society for Pain Management Nursing, and is a professional advisor to Out on a Limb, a limb loss support group. 